\title{
Analytical approximation of the transition density of a local volatility model
}

\author{
Stefano Pagliarani, Andrea Pascucci ${ }^{\dagger}$
}

April 27, 2011

\begin{abstract}
We present a simplified approach to the analytical approximation of the transition density related to a general local volatility model. The methodology is sufficiently flexible to be extended to time-dependent coefficients, multi-dimensional stochastic volatility models, degenerate parabolic PDEs related to Asian options and also to include jumps.
\end{abstract}

\section{Introduction}

Analytical approximation methods in option pricing have attracted an ever increasing interest in the last years. This is due to the demand for more sophisticated pricing models, including local, stochastic volatility and/or jumps, that generally cannot be solved in closed-form: for these models, the practical issue of the implementation is striking, in particular in the calibration and risk management processes that are typically very demanding and computationally time-consuming. The main advantage of the analytical approaches with respect to other numerical methods, such as finite-difference and Fourier inversion, is that in general the first ones are much faster and precise, at least under certain model parameter regime. Secondly, analytic formulae retain qualitative model information and preserve an explicit dependence of the results on the underlying parameters.

The application of asymptotic expansions and singular perturbation methods in mathematical finance has been studied by several authors: one of the first results was obtained by Whalley and Wilmott [31] in the study of transaction costs; in the seminal paper [18] Hagan and Woodward derived an asymptotic expansion formula for the implied volatility in a local volatility (LV) model where the volatility function can be written as the product

\footnotetext{
*Email: stefanop@math.unipd.it, Dipartimento di Matematica, Università di Padova, Via Trieste 63, 35121 Padova, Italy

${ }^{\dagger}$ Email: pascucci@dm.unibo.it, Dipartimento di Matematica, Università di Bologna, Piazza di Porta S. Donato 5, 40126 Bologna, Italy
} 
of two independent functions of time and underlying, the prototype example being the constant elasticity of variance (CEV) model; the approximation of more general one-dimensional LV models, using different deterministic and probabilistic techniques, was studied among others by Howison [22], Widdicks, Duck, Andricopoulos and Newton [32], Capriotti [5], Gatheral, Hsu, Laurence, Ouyang and Wang [15], Benhamou, Gobet and Miri [3]. A further different approach based on heat kernel expansion and the parametrix method was proposed by Corielli, Foschi and Pascucci [7], Cheng, Constantinescu, Costanzino, Mazzucato and Nistor [6], Kristensen and Mele [24].

Asymptotic methods for two-factor stochastic volatility models were studied by Hagan, Kumar, Lesniewski and Woodward [16] who introduced the SABR model; further improvements were proposed by Fouque, Papanicolaou, Sircar and Solna [14], Berestycki, Busca and Florent [4], Antonelli and Scarlatti [1]. In [25] Lesniewski introduced a geometric approach to the approximation of stochastic volatility models using the heat kernel expansion on a Riemann manifold: this approach was further developed by Hagan, Lesniewski and Woodward [17], Paulot [28], Taylor [30] and HenryLabordère who in [19] generalized the SABR to a model with mean-reverting volatility, the so-called $\lambda$-SABR.

In this paper we are mainly concerned with one-dimensional LV models of the form

$$
d S_{t}=\mu\left(t, S_{t}\right) d t+\sigma\left(t, S_{t}\right) S_{t} d W_{t}
$$

we present in this simplel framework a methodology that can be applied to multi-dimensional models, jump-diffusion models and even in the case of ultraparabolic pricing equations such as PDEs for Asian options: these and other issues are covered in the forthcoming paper [13].

The starting point of our analysis is the paper by Hagan and Woodward [18]. We develop the approach in [18] and show how it can be simplified and adapted to derive an expansion directly of the density of the underlying process, with the possibility of incorporating time-dependency and even jumps. Our main result is an expansion of the transition density $\Gamma\left(t, S_{0} ; T, S\right)$ of $S$ in (1.1) of the form

$$
\Gamma\left(t, S_{0} ; T, S\right) \approx G^{0}\left(t, S_{0} ; T, S\right)+\sum_{n=1}^{N} J_{S_{0}}^{n} G^{0}\left(t, S_{0} ; T, S\right), \quad N \geq 1,
$$

where the main term $G^{0}\left(t, S_{0} ; T, S\right)$ is the density in a suitable Black\&Scholes model, while $J_{S_{0}}^{n}$ is a differential operator containing derivatives with respect to the variable $S_{0}$ : thus the terms of the expansion (1.2) are linear combinations of Black\&Scholes sensitivities (Greeks). We show how to compute the explicit expression of the operators $J_{S_{0}}^{n}$ at any order by using an iterative algorithm that is straightforward to implement by using a symbolic computational software. 
From (1.2), we can easily derive the prices and the Greeks of plain vanilla options: for instance, for a Call option with strike $K$ and maturity $T$, we have

$$
\begin{aligned}
C\left(t, S_{0}\right) & =\int \Gamma\left(t, S_{0} ; T, S\right)(S-K)^{+} d S \\
& \approx\left(1+\sum_{n=1}^{N} J_{S_{0}}^{n}\right) \int \Gamma^{0}\left(t, S_{0} ; T, S\right)(S-K)^{+} d S \\
& =C_{\mathrm{BS}}\left(t, S_{0}\right)+\sum_{n=1}^{N} J_{S_{0}}^{n} C_{\mathrm{BS}}\left(t, S_{0}\right),
\end{aligned}
$$

where $C_{\mathrm{BS}}$ denotes a Black\&Scholes price. Formula (1.2) can also be used to price efficiently American options and other exotic derivatives. We remark that the accuracy of the approximation is independent of the smoothness of the payoff function because the operators of the expansion act directly on the transition density of the process and not on the payoff function. Another key feature of our approach is that at any order the approximated density integrates to one (cf. Remark 2.5), thus avoiding the introduction of arbitrage opportunities: for instance, the approximated option prices verify the Put-Call parity.

Finally, the smaller the time lag $T-t$ is, the faster the convergence of the expansion is. Thus, for longer maturities, it is advantageous to split the time interval in order to get a significant improvement of the accuracy. In Remark 2.4, we show how this can be done by using the expansion (1.2) and also in this case we find explicit formulae.

The layout of the paper is as follows. In Section 2 we present our main results and provide the full detail of the proof in a simplified setting. In Section 3 we carry the expansion out to order two (i.e. $N=2$ in (1.2)) for the general LV model (1.1). The numerical Section 4 investigates the effectiveness of the approximation obtained in two relevant models: in the case of a quadratic LV model we test the expansion against standard Monte Carlo approximation; secondly, we analyze more extensively the CEV model and compare ours to the earlier approximations by Hagan and Woodward [18] and by Henry-Labordère [19].

Acknowledgements. This paper is based on the talk given by the second author at the MathFinance Conference held in Frankfurt on March 14-15, 2011. The Mathematica notebook with the implementation of the formulae of this paper is available on the web site of the authors. 


\section{Analytical approximation of parabolic fundamen- tal solutions}

In this section we present the approximation technique in the simplest case of a differential operator of the form

$$
L=\frac{a(x)}{2} \partial_{x x}+\partial_{t}, \quad(t, x) \in \mathbb{R}^{2},
$$

where $a$ is a smooth function such that

$$
a_{0} \leq a(x) \leq C, \quad x \in \mathbb{R},
$$

for some positive constants $a_{0}$ and $C$. For simplicity of exposition, we only consider time-independent coefficients and refer to Section 3 for the general case. By taking the Taylor expansion of $a$ with starting point $\bar{x} \in \mathbb{R}$, we have

$$
L=L^{0}+\sum_{k=1}^{\infty} \alpha_{k}(x-\bar{x})^{k} \partial_{x x}
$$

where

$$
L^{0}=\frac{\alpha_{0}}{2} \partial_{x x}+\partial_{t}, \quad \alpha_{0}=a(\bar{x}),
$$

is the 0 -th order approximation of $L$ and

$$
\alpha_{n}=\frac{1}{2 n !} \partial_{x}^{n} a(\bar{x}), \quad n \geq 1 .
$$

We denote by $\Gamma(t, x ; T, y)$ the fundamental solution of $L$ evaluated at $(t, x)$ with pole in $(T, y)$ : from the probabilistic point of view, $\Gamma(t, x ; T, y)$ is the transition density of the solution to the SDE

$$
d X_{t}=\sqrt{a\left(X_{t}\right)} d W_{t},
$$

where $W$ is a standard Wiener process: precisely, $y \mapsto \Gamma(t, x ; T, y)$ is the density of the random variable $X_{T}^{t, x}$, where $X^{t, x}$ denotes the solution of (2.6) with initial condition $X_{t}^{t, x}=x$.

We aim to approximate $\Gamma$ by an expansion of the form

$$
\Gamma(t, x ; T, y)=\sum_{k \geq 0} G^{k}(t, x ; T, y)
$$

where the $\left(G^{k}\right)_{k>0}$ is defined recursively in terms of the solutions of a sequence of Cauchy problems. More precisely, the leading term of the expansion

$$
G^{0}(t, x ; T, y):=\frac{1}{\sqrt{2 \pi \alpha_{0}(T-t)}} \exp \left(-\frac{(x-y)^{2}}{2 \alpha_{0}(T-t)}\right), \quad x, y \in \mathbb{R}, t<T,
$$


is the fundamental solution of $L^{0}$ in (2.5). Moreover, for any $(T, y) \in \mathbb{R}^{2}$, we denote by $G^{1}(\cdot, \cdot ; T, y)$ the solution of the Cauchy problem

$$
\left\{\begin{array}{l}
L^{0} G^{1}(t, x ; T, y)+\alpha_{1}(x-\bar{x}) \partial_{x x} G^{0}(t, x ; T, y)=0, \quad t<T, x \in \mathbb{R}, \\
G^{1}(t, x ; T, y)=0, \quad x \in \mathbb{R},
\end{array}\right.
$$

and by $G^{2}(\cdot, \cdot ; T, y)$ the solution to

$$
\left\{\begin{array}{l}
L^{0} G^{2}+\alpha_{1}(x-\bar{x}) \partial_{x x} G^{1}+\alpha_{2}(x-\bar{x})^{2} \partial_{x x} G^{0}=0, \quad t<T, x \in \mathbb{R}, \\
G^{2}(t, x ; T, y)=0, \quad x \in \mathbb{R} .
\end{array}\right.
$$

In general, we put

$$
\Gamma^{N}(t, x ; T, y)=\sum_{n=0}^{N} G^{n}(t, x ; T, y), \quad N \geq 0,
$$

and we define recursively the sequence $\left(G^{n}\right)_{n \in \mathbb{N}}$ by setting

$$
\left\{\begin{array}{l}
L^{0} G^{n}+\sum_{k=1}^{n} \alpha_{k}(x-\bar{x})^{k} \partial_{x x} G^{n-k}=0, \quad t<T, x \in \mathbb{R}, \\
G^{n}(t, x ; T, y)=0, \quad x \in \mathbb{R} .
\end{array}\right.
$$

Remark 2.1. The particular choice of the Cauchy problems (2.11) comes from the fact that

$$
L^{0} \Gamma^{N}=\sum_{n=0}^{N} L^{0} G^{n}=
$$

(by (2.11))

$$
\begin{aligned}
& =-\sum_{n=0}^{N} \sum_{k=1}^{n} \alpha_{k}(x-\bar{x})^{k} \partial_{x x} G^{n-k}=-\sum_{k=1}^{N} \alpha_{k}(x-\bar{x})^{k} \partial_{x x} \sum_{n=k}^{N} G^{n-k} \\
& =-\sum_{k=1}^{N} \alpha_{k}(x-\bar{x})^{k} \partial_{x x} \Gamma^{N-k}
\end{aligned}
$$

and passing to the limit as $N \rightarrow \infty$, from (2.12) and (2.4) we get

$$
L \sum_{n=0}^{\infty} G^{n}=0 .
$$

Moreover, formally we have

$$
\Gamma^{N}(T, x ; T, y)=G^{0}(T, x ; T, y)=\delta(x-y),
$$

for any $N \geq 0$, where $\delta$ is the Dirac delta centered at the origin. 
In order to compute the explicit solution to problems (2.11), we recall some properties of the Gaussian density $\Gamma^{0}=G^{0}$ in (2.8). First of all, we shall use the following general reproduction (or semigroup) property of $\Gamma^{0}$ (cf., for instance, [27]): for any $t<s<T$ and $x, y \in \mathbb{R}$, we have

$$
\int_{\mathbb{R}} \Gamma^{0}(t, x ; s, \eta) \Gamma^{0}(s, \eta ; T, y) d \eta=\Gamma^{0}(t, x ; T, y) .
$$

Moreover we need the following elementary lemma.

Lemma 2.2. For any $x, y \in \mathbb{R}$ and $t<T$, we have

$$
\begin{aligned}
\partial_{y} \Gamma^{0}(t, x ; T, y) & =-\partial_{x} \Gamma^{0}(t, x ; T, y), \\
y \Gamma^{0}(t, x ; T, y) & =x \Gamma^{0}(t, x ; T, y)+(T-t) \alpha_{0} \partial_{x} \Gamma^{0}(t, x ; T, y) .
\end{aligned}
$$

Proof. Formula (2.15) is obvious by symmetry, while formula (2.14) follows from the identity

$$
\partial_{x} \Gamma^{0}(t, x ; T, y)=-\frac{x-y}{(T-t) \alpha_{0}} \Gamma^{0}(t, x ; T, y) .
$$

By Lemma 2.2, the partial derivative $\partial_{y}$ acts as $-\partial_{x}$ on $\Gamma^{0}(t, x ; T, y)$. Moreover, if $m_{x}$ denotes the product operator

$$
m_{x} f=x f,
$$

then, for any $c \in \mathbb{R}$, the operator $m_{y+c}$ acts as

$$
m_{x+c}+(T-t) \alpha_{0} \partial_{x}
$$

on $\Gamma^{0}(t, x ; T, y)$. More generally, we also have

$$
\begin{aligned}
(y+c)^{k} \Gamma^{0}(t, x ; T, y) & =m_{y+c}^{k} \Gamma^{0}(t, x ; T, y) \\
& =\left(m_{x+c}+(T-t) \alpha_{0} \partial_{x}\right)^{k} \Gamma^{0}(t, x ; T, y)
\end{aligned}
$$

for any $k \in \mathbb{N}$.

Proposition 2.3. The solution $G^{n}$ of problem (2.11) is given by

$$
G^{n}(t, x ; T, y)=J_{t, T, x}^{n} \Gamma^{0}(t, x ; T, y)
$$

where $J_{t, T, x}^{n}$ is a differential operator of the form

$$
J_{t, T, x}^{n}=\sum_{k=2}^{3 n} c_{n, k}(T-t)^{p_{n, k}}(x-\bar{x})^{q_{n, k}} \partial_{x}^{k}
$$


with $c_{n, k} \in \mathbb{R}, p_{k}, q_{k} \in \mathbb{N}$ such that ${ }^{1}$

$$
2 p_{n, k} \geq n,
$$

$a n d^{2}$

$$
2 p_{n, k}+q_{n, k}-k=n .
$$

The operators $J_{t, T, x}^{n}$ in (2.17) can be computed iteratively (cf. Remark 2.4): for instance, for $n=1,2$ we have

$$
\begin{aligned}
J_{t, T, x}^{1}= & \alpha_{1}(T-t)(x-\bar{x}) \partial_{x}^{2}+\frac{\alpha_{1} \alpha_{0}}{2}(T-t)^{2} \partial_{x}^{3} \\
J_{t, T, x}^{2}= & \frac{\alpha_{2}}{2}(T-t)\left(\alpha_{0}(T-t)+2(x-\bar{x})^{2}\right) \partial_{x}^{2} \\
& +\left(\alpha_{1}^{2}+\alpha_{0} \alpha_{2}\right)(T-t)^{2}(x-\bar{x}) \partial_{x}^{3} \\
& +\left(\frac{1}{6}(T-t)^{3} \alpha_{0}\left(3 \alpha_{1}^{2}+2 \alpha_{0} \alpha_{2}\right)+\frac{1}{2}(T-t)^{2} \alpha_{1}^{2}(x-\bar{x})^{2}\right) \partial_{x}^{4} \\
& +\frac{\alpha_{0} \alpha_{1}^{2}}{2}(T-t)^{3}(x-\bar{x}) \partial_{x}^{5}+\frac{\alpha_{0}^{2} \alpha_{1}^{2}}{8}(T-t)^{4} \partial_{x}^{6} .
\end{aligned}
$$

Proof. By the standard representation formula for solutions to the nonhomogeneous parabolic Cauchy problem with null final condition, we have

$G^{1}(t, x ; T, y)=\int_{t}^{T} \int_{\mathbb{R}} \Gamma^{0}(t, x ; s, \eta) \alpha_{1}(\eta-\bar{x}) \partial_{\eta \eta} \Gamma^{0}(s, \eta ; T, y) d \eta d s=$

(using notation (2.16))

$$
=\alpha_{1} \int_{t}^{T} \int_{\mathbb{R}} m_{\eta-\bar{x}} \Gamma^{0}(t, x ; s, \eta) \partial_{\eta \eta} \Gamma^{0}(s, \eta ; T, y) d \eta d s=
$$

(by $(2.15)$ )

$$
=\alpha_{1} \int_{t}^{T}\left(m_{x-\bar{x}}+(s-t) \alpha_{0} \partial_{x}\right) \int_{\mathbb{R}} \Gamma^{0}(t, x ; s, \eta) \partial_{\eta \eta} \Gamma^{0}(s, \eta ; T, y) d \eta d s=
$$

(by parts)

$$
=\alpha_{1} \int_{t}^{T}\left(m_{x-\bar{x}}+(s-t) \alpha_{0} \partial_{x}\right) \int_{\mathbb{R}} \partial_{\eta \eta} \Gamma^{0}(t, x ; s, \eta) \Gamma^{0}(s, \eta ; T, y) d \eta d s=
$$

(by $(2.14)$ )

$$
=\alpha_{1} \int_{t}^{T}\left(m_{x-\bar{x}}+(s-t) \alpha_{0} \partial_{x}\right) \partial_{x x} \int_{\mathbb{R}} \Gamma^{0}(t, x ; s, \eta) \Gamma^{0}(s, \eta ; T, y) d \eta d s=
$$

\footnotetext{
${ }^{1}$ By (2.18), the approximation is better for shorter times and larger $n$.

${ }^{2} \mathrm{By}(2.19)$, there is a sort of parabolic homogeneity and the damping effect of $T-t$ and $x-\bar{x}$ becomes stronger as the order of the approximation increases.
} 
(by the reproduction property (2.13))

$$
=\alpha_{1} \int_{t}^{T}\left(m_{x-\bar{x}}+(s-t) \alpha_{0} \partial_{x}\right) d s \partial_{x x} \Gamma^{0}(t, x ; T, y) .
$$

Thus we have

$$
J_{t, T, x}^{1}=\alpha_{1} \int_{t}^{T}\left(m_{x-\bar{x}}+(s-t) \alpha_{0} \partial_{x}\right) d s \partial_{x x}
$$

and integrating in $s$ we get (2.20).

Concerning $G^{2}$, we have

$$
G^{2}(t, x ; T, y)=I_{1}+I_{2}
$$

where, proceeding as before,

$$
\begin{aligned}
I_{1} & =\alpha_{2} \int_{t}^{T} \int_{\mathbb{R}} \Gamma^{0}(t, x ; s, \eta)(\eta-\bar{x})^{2} \partial_{\eta \eta} \Gamma^{0}(s, \eta ; T, y) d \eta d s \\
& =\alpha_{2} \int_{t}^{T}\left(m_{x-\bar{x}}+(s-t) \alpha_{0} \partial_{x}\right)^{2} \partial_{x x} \int_{\mathbb{R}} \Gamma^{0}(t, x ; s, \eta) \Gamma^{0}(s, \eta ; T, y) d \eta d s \\
& =\alpha_{2} \int_{t}^{T}\left(m_{x-\bar{x}}+(s-t) \alpha_{0} \partial_{x}\right)^{2} d s \partial_{x x} \Gamma^{0}(t, x ; T, y),
\end{aligned}
$$

and

$$
\begin{aligned}
I_{2} & =\alpha_{1} \int_{t}^{T} \int_{\mathbb{R}} \Gamma^{0}(t, x ; s, \eta)(\eta-\bar{x}) \partial_{\eta \eta} G^{1}(s, \eta ; T, y) d \eta d s \\
& =\alpha_{1} \int_{t}^{T}\left(m_{x-\bar{x}}+(s-t) \alpha_{0} \partial_{x}\right) \partial_{x x} \int_{\mathbb{R}} \Gamma^{0}(t, x ; s, \eta) J_{s, T, \eta}^{1} \Gamma^{0}(s, \eta ; T, y) d \eta d s \\
& =\alpha_{1} \int_{t}^{T}\left(m_{x-\bar{x}}+(s-t) \alpha_{0} \partial_{x}\right) \partial_{x x} \widetilde{J}_{s, T, x}^{1} \int_{\mathbb{R}} \Gamma^{0}(t, x ; s, \eta) \Gamma^{0}(s, \eta ; T, y) d \eta d s \\
& =\alpha_{1} \int_{t}^{T}\left(m_{x-\bar{x}}+(s-t) \alpha_{0} \partial_{x}\right) \partial_{x x} \widetilde{J}_{s, T, x}^{1} d s \Gamma^{0}(t, x ; T, y),
\end{aligned}
$$

with

$$
\widetilde{J}_{s, T, x}^{1}=\alpha_{1}(T-s)\left(m_{x-\bar{x}}+(s-t) \alpha_{0} \partial_{x}\right) \partial_{x}^{2}+\frac{\alpha_{1} \alpha_{0}(T-s)^{2}}{2} \partial_{x}^{3} .
$$

In conclusion we have

$$
\begin{aligned}
J_{t, T, x}^{2}= & \alpha_{2} \int_{t}^{T}\left(m_{x-\bar{x}}+(s-t) \alpha_{0} \partial_{x}\right)^{2} d s \partial_{x x} \\
& +\alpha_{1} \int_{t}^{T}\left(m_{x-\bar{x}}+(s-t) \alpha_{0} \partial_{x}\right) \partial_{x x} \widetilde{J}_{s, T, x}^{1} d s
\end{aligned}
$$

and integrating in $s$ we get formula (2.21). Eventually, the general expression (2.17) can be proved by induction. 
Remark 2.4. The proof of Proposition 2.3 provides a constructive algorithm that can be used to compute higher order approximations iteratively. In particular this can be done by means of a symbolic computation software to get the explicit expression of the operators $J_{t, T, x}^{n}$ in (2.17): for instance, using Mathematica one can find the expression of $J_{t, T, x}^{1}$ in $(2.22)$ or $J_{t, T, x}^{2}$ in (2.23) by writing just one line of code. Moreover, since the derivatives of the Gaussian density $\Gamma^{0}$ can be expressed in terms of Hermite polynomials, the computation of the terms of the expansion is extremely fast.

According to (2.7), the $N$-th order approximation of $\Gamma$ is given by

$$
\Gamma^{N}(t, x ; T, y)=\sum_{n=0}^{N} G^{n}(t, x ; T, y) .
$$

By Proposition 2.3 we have

$$
\Gamma^{N}(t, x ; T, y)=\Gamma^{0}(t, x ; T, y)+\mathcal{A}_{t, T, x}^{N} \Gamma^{0}(t, x ; T, y),
$$

where $\Gamma^{0} \equiv G^{0}$ is the Gaussian density in (2.8) and $\mathcal{A}_{t, T, x}^{N}$ is the differential operator

$$
\mathcal{A}_{t, T, x}^{N}=\sum_{n=1}^{N} J_{t, T, x}^{n}, \quad N \geq 1 .
$$

Remark 2.5. From formula (2.24), we infer that

$$
\int_{\mathbb{R}} \Gamma^{N}(t, x ; T, y) d y=\left(1+\mathcal{A}_{t, T, x}^{N}\right) \int_{\mathbb{R}} \Gamma^{0}(t, x ; T, y) d y=1 .
$$

Thus, a general property of the approximated density $\Gamma^{N}$ is that it integrates to one.

More generally, a nice feature of the approximation formula (2.24) is that $\mathcal{A}_{t, T, x}^{N}$ acts as a differential operator in the variable $x$ (starting point of the underlying stochastic process). As a consequence, the price of an option with payoff function $\varphi$ is simply given by

$$
\begin{aligned}
C(t, x) & =\int \Gamma(t, x ; T, y) \varphi(y) d y \\
& \approx\left(1+\mathcal{A}_{t, T, x}^{N}\right) \int_{\mathbb{R}} \Gamma^{0}(t, x ; T, y) \varphi(y) d y=C_{\mathrm{BS}}(t, x)+\mathcal{A}_{t, T, x}^{N} C_{\mathrm{BS}}(t, x),
\end{aligned}
$$

where $C_{\mathrm{BS}}$ denotes the option price in a Gaussian model (Black\&Scholes). The financial interpretation is that the approximated price is given by the B\&S price plus higher order terms defined by a linear combination of Greeks of the option. 
The approximation formula for the density is useful to price American options and other exotic derivatives. The Greeks/sensitivities can be easily computed as well: indeed, notice that

$$
\partial_{x} C(t, x) \approx \partial_{x} C_{\mathrm{BS}}(t, x)+\partial_{x} \mathcal{A}_{t, T, x}^{N} C_{\mathrm{BS}}(t, x)
$$

so that the problem of the irregularity of the payoff function (typical of Monte Carlo approximation) is overcome.

Remark 2.6. Even if not stated explicitly, the approximation result of Proposition 2.3 depends on the choice of starting point $\bar{x}$ of the Taylor expansion. With the specification $\bar{x}=x$, we get a particularly simple expression of the operators $J_{t, x, T}^{n}$ in (2.20)-(2.21):

$$
\begin{aligned}
J_{t, T, x}^{1}= & \frac{\alpha_{1} \alpha_{0}(T-t)^{2}}{2} \partial_{x}^{3}, \\
J_{t, T, x}^{2}= & \frac{\alpha_{2} \alpha_{0}}{2}(T-t)^{2} \partial_{x}^{2}+\frac{\alpha_{0}}{6}\left(2 \alpha_{0} \alpha_{2}+3 \alpha_{1}^{2}\right)(T-t)^{3} \partial_{x}^{4}+\frac{\alpha_{0}^{2} \alpha_{1}^{2}}{8}(T-t)^{4} \partial_{x}^{6}, \\
J_{t, T, x}^{3}= & \frac{\alpha_{0}}{3}\left(4 \alpha_{1} \alpha_{2}+3 \alpha_{0} \alpha_{3}\right)(T-t)^{3} \partial_{x}^{3} \\
& +\frac{\alpha_{0}}{12}\left(6 \alpha_{1}^{3}+16 \alpha_{0} \alpha_{1} \alpha_{2}+3 \alpha_{0}^{2} \alpha_{3}\right)(T-t)^{4} \partial_{x}^{5} \\
& +\frac{\alpha_{0}^{2} \alpha_{1}}{12}\left(3 \alpha_{1}^{2}+2 \alpha_{0} \alpha_{2}\right)(T-t)^{5} \partial_{x}^{7}+\frac{\alpha_{0}^{3} \alpha_{1}^{3}}{48}(T-t)^{6} \partial_{x}^{9} .
\end{aligned}
$$

Other specifications of $\bar{x}$ can be used to minimize the approximation error.

Remark 2.7. The approximation can be further improved by using the reproduction property (2.13) and exploiting the fact that the approximation is more efficient for short maturities because of the presence of powers of $T-t$ in the coefficients of the expansion. We first note that, integrating by parts and by (2.14), for $J_{t, T, x}^{1}$ as in (2.26) we have

$$
\begin{aligned}
& \int_{\mathbb{R}} \Gamma^{0}(t, x ; s, \eta)\left(1+J_{s, T, \eta}^{1}\right) \Gamma^{0}(s, \eta ; T, y) d \eta= \\
& =\left(1+J_{s, T, x}^{1}\right) \int_{\mathbb{R}} \Gamma^{0}(t, x ; s, \eta) \Gamma^{0}(s, \eta ; T, y) d \eta=
\end{aligned}
$$

(by the reproduction property)

$$
=\left(1+J_{s, T, x}^{1}\right) \Gamma^{0}(t, x ; T, y) .
$$

Then, for any $t<s<T$ and $x, y \in \mathbb{R}$, we have

$$
\begin{aligned}
\Gamma(t, x ; T, y) & =\int_{\mathbb{R}} \Gamma(t, x ; s, \eta) \Gamma(s, \eta ; T, y) d \eta \\
& \approx \int_{\mathbb{R}}\left(1+J_{t, s, x}^{1}\right) \Gamma^{0}(t, x ; s, \eta)\left(1+J_{s, T, \eta}^{1}\right) \Gamma^{0}(s, \eta ; T, y) d \eta \\
& =\left(1+J_{t, s, x}^{1}\right) \int_{\mathbb{R}} \Gamma^{0}(t, x ; s, \eta)\left(1+J_{s, T, \eta}^{1}\right) \Gamma^{0}(s, \eta ; T, y) d \eta=
\end{aligned}
$$


(by $(2.27))$

$$
=\left(1+J_{t, s, x}^{1}\right)\left(1+J_{s, T, x}^{1}\right) \Gamma^{0}(t, x ; T, y) .
$$

In general, for any $t<s_{1}<\cdots<s_{N}<T$ we have

$$
\Gamma(t, x ; T, y) \approx\left(1+J_{t, s_{1}, x}^{1}\right)\left(1+J_{s_{1}, s_{2}, x}^{1}\right) \cdots\left(1+J_{s_{N}, T, x}^{1}\right) \Gamma^{0}(t, x ; T, y),
$$

that is easily computable and may improve the approximation for long maturities.

\section{Applications to local volatility models}

We now consider the more general dynamics of a LV model in the riskneutral measure

$$
d S_{t}=r S_{t} d t+\sigma\left(t, S_{t}\right) S_{t} d W_{t}
$$

where $\sigma(t, S)$ is a suitably regular function such that

$$
\sigma_{1} \leq \sigma(t, S) \leq \sigma_{2}, \quad t>0, S>0,
$$

for some positive constants $\sigma_{1}, \sigma_{2}$. The pricing Cauchy problem is

$$
\begin{cases}\mathcal{L} C(t, S)=0, & t<T, S>0, \\ C(T, S)=\varphi(S), & S>0,\end{cases}
$$

where

$$
\mathcal{L}=\frac{\sigma^{2}(t, S) S^{2}}{2} \partial_{S S}+r S \partial_{S}+\partial_{t}-r
$$

The function

$$
u(t, x)=e^{r(T-t)} C\left(t, e^{x}\right)
$$

solves the parabolic Cauchy problem

$$
\left\{\begin{array}{l}
L u(t, x)=0, \quad t<T, x \in \mathbb{R}, \\
u(T, x)=\varphi\left(e^{x}\right), \quad x \in \mathbb{R},
\end{array}\right.
$$

where

$$
L=\frac{a(t, x)}{2}\left(\partial_{x x}-\partial_{x}\right)+r \partial_{x}+\partial_{t}, \quad(t, x) \in \mathbb{R}^{2},
$$

and

$$
a(t, x)=\sigma^{2}\left(t, e^{x}\right) .
$$

For sake of simplicity, we just consider the case of time-independent volatility

$$
\sigma(t, S)=\sigma(S)
$$


so that $a(t, x)=a(x)$. This assumption is not restrictive: in the timedependent case, the method does not present any further difficulty, even if it leads to longer computations. Hereafter, we fix $\bar{x} \in \mathbb{R}$ and we use the notation

$$
\alpha_{0}=a(\bar{x}) \equiv \sigma^{2}\left(e^{\bar{x}}\right), \quad \alpha_{n}=\frac{1}{2 n !} \partial_{x}^{n} a(\bar{x}), \quad n \geq 1 .
$$

By using the method presented in Section 2, we get the following approximation formula for the density of $S$. We omit the proof since it is based on the same technique presented in Section 2.

Proposition 3.1. Let $\Gamma(0, S ; T, y)$ be the transition density of $S_{T}^{0, S}$ in (3.28). Then we have

$$
\Gamma(0, S ; T, y) \approx \frac{1}{y} \Gamma^{N}(0, \log S ; T, \log y)
$$

where

$$
\Gamma^{N}(0, x ; T, z)=\left(1+\mathcal{A}_{0, T, x}^{N}\right) \Gamma^{0}(0, x ; T, z),
$$

and

$$
\Gamma^{0}(t, x ; T, z)=\frac{1}{\sqrt{2 \pi \alpha_{0}(T-t)}} \exp \left(-\frac{\left(x-z+(T-t)\left(r+\frac{\alpha_{0}}{2}\right)\right)^{2}}{2 \alpha_{0}(T-t)}\right)
$$

is the fundamental solution of the operator

$$
L^{0}=\frac{\alpha_{0}}{2}\left(\partial_{x x}-\partial_{x}\right)+r \partial_{x}+\partial_{t}, \quad(t, x) \in \mathbb{R}^{2} .
$$

Moreover $\mathcal{A}_{0, T, x}^{N}$ is the differential operator in (2.25), whose explicit expression can be found iteratively proceeding as in the proof of Proposition 2.3: in particular, for $N=2$ we have

$$
J_{0, T, x}^{i}=\sum_{n=1}^{3 i} f_{n}^{i}(x) \partial_{x}^{n}, \quad i=1,2,
$$

where

$$
\begin{aligned}
f_{1}^{1}(x)= & \frac{1}{4} \alpha_{1} T\left(-2 r T-4 x+4 \bar{x}+T \alpha_{0}\right), \\
f_{2}^{1}(x)= & \frac{1}{4} \alpha_{1} T\left(2 r T+4 x-4 \bar{x}-3 T \alpha_{0}\right), \\
f_{3}^{1}(x)= & \frac{1}{2} \alpha_{0} \alpha_{1} T^{2} \\
f_{1}^{2}(x)= & -\frac{T}{12}\left(4 r^{2} T^{2} \alpha_{2}+12(x-\bar{x})^{2} \alpha_{2}+T^{2} \alpha_{0}\left(\alpha_{1}^{2}+\alpha_{0} \alpha_{2}\right)\right. \\
& +6 T\left(\alpha_{0} \alpha_{2}-(x-\bar{x})\left(\alpha_{1}^{2}+\alpha_{0} \alpha_{2}\right)\right)-2 r T\left(6(-x+\bar{x}) \alpha_{2}\right. \\
& \left.\left.+T\left(\alpha_{1}^{2}+2 \alpha_{0} \alpha_{2}\right)\right)\right),
\end{aligned}
$$




$$
\begin{aligned}
f_{2}^{2}(x)= & \frac{1}{96} T\left(3 T^{3} \alpha_{0}^{2} \alpha_{1}^{2}+96(x-\bar{x})^{2} \alpha_{2}+4 r^{2} T^{2}\left(3 T \alpha_{1}^{2}+8 \alpha_{2}\right)\right. \\
& +8 T^{2} \alpha_{0}\left((7-3 x+3 \bar{x}) \alpha_{1}^{2}+5 \alpha_{0} \alpha_{2}\right) \\
& +12 r T\left(-T\left(4-4 x+4 \bar{x}+T \alpha_{0}\right) \alpha_{1}^{2}-8\left(-x+\bar{x}+T \alpha_{0}\right) \alpha_{2}\right) \\
& \left.+48 T\left(\alpha_{0} \alpha_{2}+(x-\bar{x})\left((-3+x-\bar{x}) \alpha_{1}^{2}-3 \alpha_{0} \alpha_{2}\right)\right)\right), \\
f_{3}^{2}(x)= & \frac{1}{48} T^{2}\left(-\left(12 r^{2} T^{2}+48(-1+x-\bar{x})(x-\bar{x})\right.\right. \\
& \left.+48 T(1-x+\bar{x}) \alpha_{0}+9 T^{2} \alpha_{0}^{2}-8 r T\left(2-6 x+6 \bar{x}+3 T \alpha_{0}\right)\right) \alpha_{1}^{2} \\
& \left.+16 \alpha_{0}\left(2 r T+3 x-3 \bar{x}-2 T \alpha_{0}\right) \alpha_{2}\right), \\
f_{4}^{2}(x)= & \frac{1}{96} T^{2}\left(3 \left(4(r T+2 x-2 \bar{x})^{2}+4 T(4-5 r T-10 x+10 \bar{x}) \alpha_{0}\right.\right. \\
& \left.\left.+13 T^{2} \alpha_{0}^{2}\right) \alpha_{1}^{2}+32 T \alpha_{0}^{2} \alpha_{2}\right), \\
f_{5}^{2}(x)= & \frac{1}{8} T^{3} \alpha_{0}\left(2 r T+4 x-4 \bar{x}-3 T \alpha_{0}\right) \alpha_{1}^{2}, \\
f_{6}^{2}(x)= & \frac{1}{8} T^{4} \alpha_{0}^{2} \alpha_{1}^{2} .
\end{aligned}
$$

Next, we give a second order approximation formula for the price of a Call option. Precisely, let $C\left(t, S_{t}\right)$ denote the price at time $t$ of a Call option with strike $K$ and maturity $T$, written on an asset with dynamics given by (3.28) with time-independent volatility (3.32). According to Proposition 3.1 , we have the following

Corollary 3.2 (Call price). The second order approximation of $C\left(t, S_{t}\right)$ is given by $u\left(t, \log S_{t}\right)$ where

$$
u(t, x)=\left(1+J_{t, T, x}^{1}+J_{t, T, x}^{2}\right) C_{B S}(t, x) .
$$

In (3.35), $J_{t, T, x}^{i}, i=1,2, i s^{3}$ as in (3.34) and $C_{B S}$ is the standard Black6SCholes price function (expressed in terms of log-price of the asset)

$$
\begin{aligned}
C_{B S}(t, x) & =e^{-r(T-t)} \int_{\mathbb{R}} \frac{1}{y} \Gamma^{0}(t, x ; T, \log y)(y-K)^{+} d y \\
& =e^{x} \mathcal{N}\left(d_{1}\right)-e^{-r(T-t)} K \mathcal{N}\left(d_{2}\right),
\end{aligned}
$$

with

$$
d_{1}=\frac{2 x-2 \log K+(T-t)\left(2 r+\alpha_{0}\right)}{2 \sqrt{(T-t) \alpha_{0}}}, \quad d_{2}=d_{1}-\sqrt{\alpha_{0}(T-t)} .
$$

More explicitly, putting $\bar{x}=x$ and $t=0$, we have

$$
u(0, x)=C_{B S}(0, x)+\frac{K e^{-\frac{\left(-2(r T+x)+T \alpha_{0}+2 \log K\right)^{2}}{8 T \alpha_{0}}}}{\sqrt{2 \pi T \alpha_{0}}}\left(Q_{1}(x)+Q_{2}(x)\right)
$$

\footnotetext{
${ }^{3}$ Due to time-homogeneity we have $J_{t, T, x}^{i}=J_{0, T-t, x}^{i}$.
} 
where

$$
Q_{1}(x)=-\frac{T \alpha_{1}(x-\log K)}{2},
$$

and

$$
\begin{aligned}
Q_{2}(x)= & \frac{1}{96 \alpha_{0}^{2}}\left(12 x^{4} \alpha_{1}^{2}-8 r T x\left(-3 x^{2}+T \alpha_{0}\right) \alpha_{1}^{2}+4 r^{2} T^{2}\left(3 x^{2}+T \alpha_{0}\right) \alpha_{1}^{2}\right. \\
& -T^{2} \alpha_{0}^{2}\left(\left(12+T \alpha_{0}\right) \alpha_{1}^{2}-16 \alpha_{0} \alpha_{2}\right) \\
& -T x^{2} \alpha_{0}\left(-3\left(8+T \alpha_{0}\right) \alpha_{1}^{2}+32 \alpha_{0} \alpha_{2}\right) \\
& -\log K\left(-24 r^{2} T^{2} x \alpha_{1}^{2}-48 x^{3} \alpha_{1}^{2}+8 r T\left(-9 x^{2}+T \alpha_{0}\right) \alpha_{1}^{2}\right. \\
& -2 T x \alpha_{0}\left(3\left(8+T \alpha_{0}\right) \alpha_{1}^{2}-32 \alpha_{0} \alpha_{2}\right) \\
& -\log K\left(3\left(4 r^{2} T^{2}+24 r T x+24 x^{2}-T \alpha_{0}\left(8+T \alpha_{0}\right)\right) \alpha_{1}^{2}\right. \\
& \left.\left.\left.-32 T \alpha_{0}^{2} \alpha_{2}+12 \alpha_{1}^{2} \log K(-2 r T-4 x+\log K)\right)\right)\right)
\end{aligned}
$$

\section{Numerical tests}

In this section we test the performance of the analytical approximation formulae presented in the previous sections in the context of one-dimensional local volatility (LV) models. Let us remark explicitly that when the underlying asset is modeled by a geometric Brownian motion, then our approximation reduces to the standard Black\&Scholes formula. For more complicated models, we obtain accurate approximations of densities and prices for a reasonably wide range of parameters. We first consider a standard quadratic LV model and then we analyze in more details the well-known constant elasticity of variance $(\mathrm{CEV})$ model.

We assume that $S$ solves the SDE (3.28) with

$$
\sigma(t, S)=\sigma_{0} \sqrt{1+(S-a)^{2}}
$$

where $\sigma_{0}, a \in \mathbb{R}_{+}$. In the following experiment we compare the second, third and fourth order approximation of the Call price with an accurate Monte Carlo (MC) simulation. In Figure 1 the dotted, dot-dashed and dashed lines represent the relative errors of the (second, third and fourth order, respectively) approximated Call prices, for maturities $T=0.25$ (left) and $T=1$ (right). The values of the parameters are

$$
K=1, \quad r=5 \%, \quad \sigma_{0}=20 \%, \quad a=1,
$$

and the initial price $S_{0}$ ranges from 0.5 to 1.5. A 200-steps Euler discretization of the SDEs and a MC with 1.000.000 simulations have been used. We marked by gray (light gray) the confidence regions where prices differ no more than 2 (2.57) standard deviations from the simulated MC prices: in 


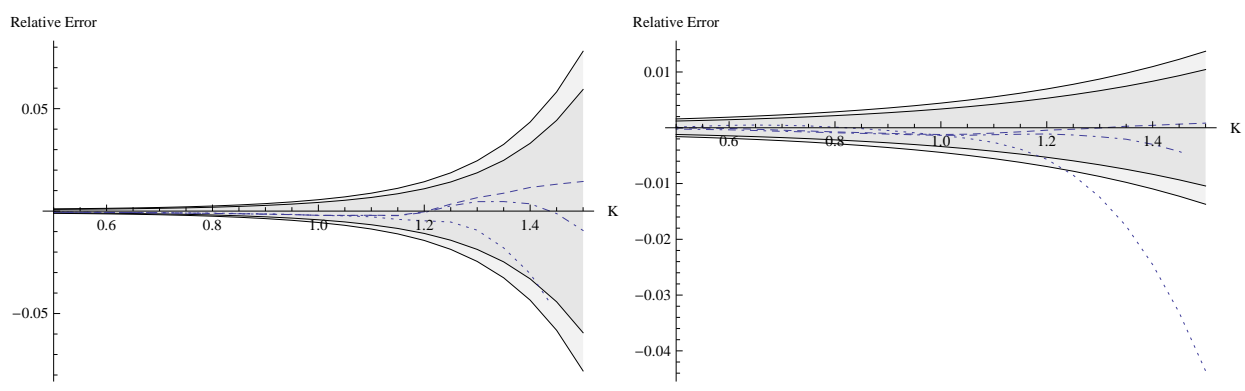

Figure 1: Relative errors of the second (dotted line), third (dot-dashed line) and fourth (dashed line) order approximated Call prices (compared with MC prices). The $95 \%$ and $99 \%$ MC confidence regions are marked by gray and light gray respectively. A 200-steps Euler discretization of the SDEs and a MC with 1.000.000 simulations have been used. The parameters are as in (4.37) and $T=0.25$ (left) and $T=1$ (right)
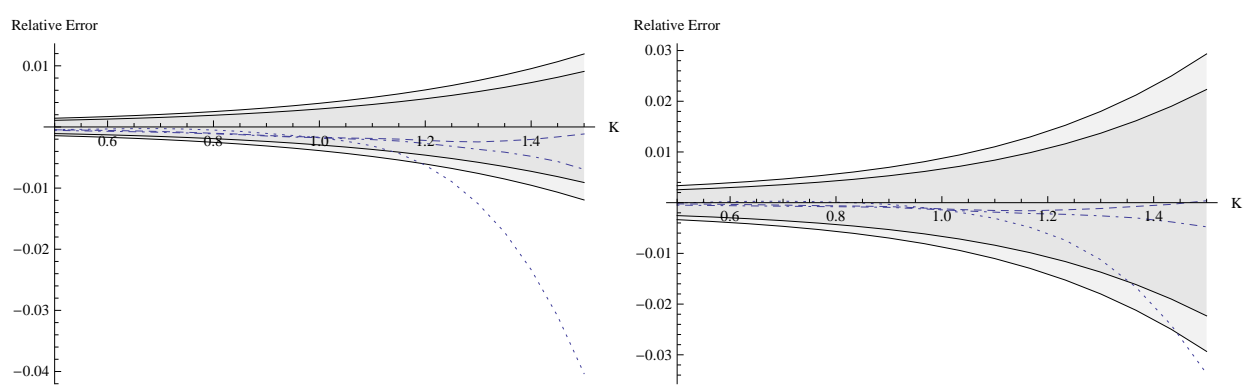

Figure 2: Relative errors of the second (dotted line), third (dot-dashed line) and fourth (dashed line) order approximated Call prices (compared with MC prices). The $95 \%$ and $99 \%$ MC confidence regions are marked by gray and light gray respectively. A 500-steps Euler discretization of the SDEs and a MC with 1.000.000 simulations have been used. The parameters are as in (4.37) and $T=2$ (left) and $T=3$ (right)

other words, according to the MC approximation, the true price belongs to the gray (light gray) region with probability $95 \%$ (99\%).

In Figure 2 we repeat the same experiment for longer maturities, $T=2$ (left) and $T=3$ (right): in this case a 500-steps Euler discretization has been used.

Next we consider the classical CEV model introduced by Cox [8]:

$$
d S_{t}=\mu S_{t} d t+\sigma S_{t}^{\beta} d W_{t} .
$$

We are interested in this particular LV model because the difficulties in the numerical approximation of the CEV model are similar to those of the SABR model that is its stochastic volatility counterpart and is widely used to price interest rate options. These difficulties are mainly caused by the fact that if $\beta \in] 0,1[$ the point 0 is an attainable state for $S$ (see, for instance, Lindsay 
and Brecher [26]). As soon as $S$ reaches zero, we have to keep it equal zero (absorbing boundary): under this condition, Delbaen and Shirakawa [10] proved the existence of a unique equivalent martingale measure under which the dynamics of $S$ are as in (4.38) with $\mu=r$ ( $r$ denotes the risk-free rate); moreover the arbitrage free price at time $t$, of the option which pays $\varphi\left(S_{T}\right)$ at time $T$, is given by

$$
C(t, S)=e^{-r(T-t)} E\left[\varphi\left(S_{T}^{t, S}\right)\right] .
$$

Notice that, if we assume the origin as a reflecting boundary then the CEV model admits arbitrage opportunities (cf. [10] or [21]).

The density of $S$ can be expressed explicitly in terms of special functions: precisely, for $r=0$ we have

$$
\Gamma(t, S ; T, y)=\frac{\sqrt{y} S^{\frac{1}{2}-2 \beta} e^{-\frac{S^{2(1-\beta)}+y^{2(1-\beta)}}{2(1-\beta)^{2} \sigma^{2}(T-t)}}}{(1-\beta) \sigma^{2}(T-t)} I_{\frac{1}{2(1-\beta)}}\left(\frac{(S y)^{1-\beta}}{(1-\beta)^{2} \sigma^{2}(T-t)}\right),
$$

where $I_{\nu}(x)$ is the modified Bessel function of the first kind defined by

$$
I_{\nu}(x)=\left(\frac{x}{2}\right)^{\nu} \sum_{k=0}^{\infty} \frac{x^{2 k}}{2^{2 k} k ! \mathrm{G}(\nu+k+1)}
$$

and $\mathrm{G}$ is the Gamma function. For the case $r \neq 0$, we refer for instance to Davydov and Linetsky [9].

More explicit results on the precise asymptotic behaviour of $\Gamma$ at boundary points have been recently proved by Ekström and Tysk [12]: in the case $r=0$ and $\beta \in[1 / 2,1[$, they prove that

$$
y \mapsto \Gamma(t, S ; T, y) \sim y^{1-2 \beta} \quad \text { as } y \rightarrow 0^{+} .
$$

Notice that, due to absorption, the law of $S_{T}^{t, S}$ has a Dirac delta component at the origin and consequently the function $\Gamma$ in (4.40) is not a density in the standard sense because

$$
\int_{0}^{+\infty} \Gamma(t, S ; T, y) d y<1
$$

and part of the mass is concentrated at the origin. In general this fact is difficulty captured by heat kernel expansions based on Gaussian functions, such as those proposed by Hagan and Woodward [18], Henry-Labordère [20], Paulot [28], Gatheral, Hsu, Laurence, Ouyang and Wang [15], Taylor [30] and also by our expansion presented in the previous sections. For this reason, alternative approximations were proposed by Doust [11], Barjaktarevic and Rebonato [2] in the more general setting of the SABR model. 


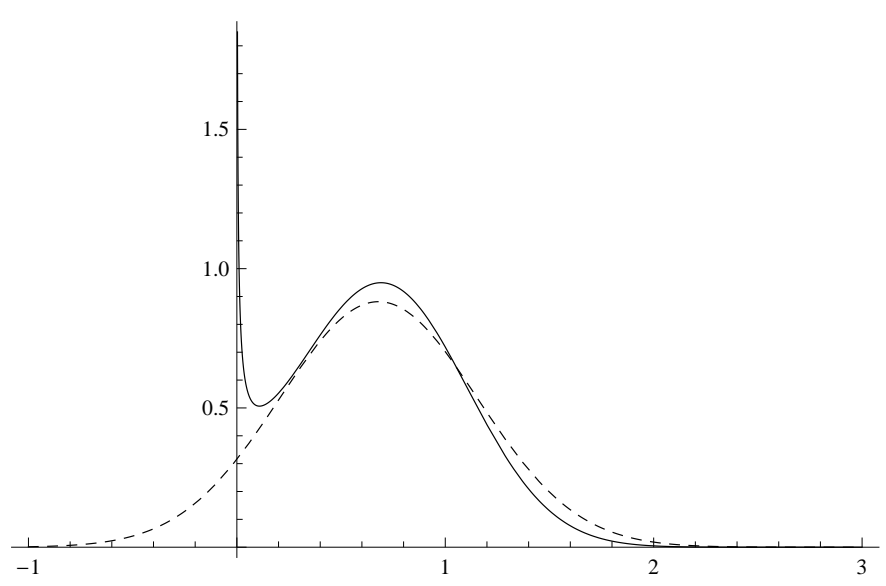

Figure 3: CEV (solid line) and Gaussian (dashed line) densities

Remark 4.1. It is not restrictive to assume, as we shall systematically do in the sequel, that $S_{0}=1$ : indeed, we can always rescale the CEV equation by setting $X_{t}=\frac{S_{t}}{S_{0}}$ and we get

$$
d X_{t}=r X_{t} d t+\sigma_{I} X_{t}^{\beta} d W_{t}
$$

where $\sigma_{I}=\sigma S_{0}^{\beta-1}$ is the so-called CEV normalized volatility. Even in the case of interest rate models where $S$ represents an interest rate with values of the order of $1 \%$, typically $\sigma_{I}$ ranges from $10 \%$ to $50 \%$.

The relationship between CEV stochastic equations and PDEs was established by Janson and Tysk [23] who proved a Feynman-Kac type theorem: precisely, they showed that, if the payoff function $\varphi$ is continuous and polynomially bounded (i.e. $|\varphi(S)| \leq C\left(1+S^{m}\right.$ ) for some $C$ and $m$ ), then the function $C$ in (4.39) is the unique, polynomially bounded classical solution to problem

$$
\begin{cases}\mathcal{L}_{\beta} C(t, S)=0, & (t, S) \in] 0, T[\times] 0,+\infty[ \\ C(T, S)=\varphi(S), & S>0, \\ C(t, 0)=e^{-r(T-t)} \varphi(0), & t \in] 0, T[\end{cases}
$$

where

$$
\mathcal{L}_{\beta}=\frac{\sigma^{2} S^{2 \beta}}{2} \partial_{S S}+r S \partial_{S}+\partial_{t}+r, \quad S>0 .
$$

Applying the 2nd-order approximation result of Section 2 directly to problem (4.41), we get exactly the classical Hagan-Woodward [18] formula for Call prices: notice that the true density of $S_{T}$ is supported on $\mathbb{R}_{+}$but it is approximated by using a Gaussian density with support on $\mathbb{R}$ (cf. Figure 3). Indeed, except for the case $\beta=0$, the pricing operator $\mathcal{L}_{\beta}$ does not 
satisfy the uniform parabolicity condition (3.29): thus, for moderately large values of $\sigma$ and for long maturities, we have a "loss of mass" of the HaganWoodward (HW) approximated density on $\mathbb{R}_{+}$and consequently, as it has been recognized by several authors, the HW approximation may become inaccurate.

To partially fix this problem, we suggest to first perform the change of variable $S=e^{x}$ to transform the Cauchy problem (4.41), posed on the semi-strip $] 0, T\left[\times \mathbb{R}_{+}\right.$, into problem

$$
\begin{cases}L_{\beta} u(t, x)=0, & (t, x) \in] 0, T[\times \mathbb{R}, \\ u(T, s)=\varphi\left(e^{x}\right), & x \in \mathbb{R},\end{cases}
$$

with

$$
L_{\beta}=\frac{\sigma^{2} e^{2(\beta-1) x}}{2}\left(\partial_{x x}-\partial_{x}\right)+r \partial_{x}+\partial_{t}+r .
$$

Since $L_{\beta}$ is defined on the entire real axis, it seems more natural to use of the heat kernel expansion to approximate its fundamental solution; once we have the approximation, we go back to the original variables to estimate the density of $S$ : we call this procedure the Log-approximation of the CEV density. The idea is that, while the HW approximation shifts part of the mass into the negative real axis, on the contrary the Log-approximation tries to mimic the delta at zero by concentrating part of the mass close to the origin. As we shall see later, the Log-approximation gives rather satisfactory results when $\beta$ is not too close to zero, that is when the CEV model is not close to the normal one.

Figure 4 shows the graph of the CEV density

$$
y \mapsto \Gamma\left(0, S_{0} ; T, y\right)
$$

in (4.40) with $S_{0}=1, \beta=\frac{2}{3}, \sigma=0.5, r=0$ and maturities $T=1,10,20,30$. For comparison we also plot the approximation by Henry-Labordère (cf. [20], p.132), the HW approximation and the 4th-order Log-approximation of $\Gamma$. In Figure 5 we consider the case $\beta=\frac{1}{3}$.

Notice that the LH, HW and Log approximations cannot reproduce the delta component of the CEV process at the origin and can take negative values. On the other hand, the integral over $\mathbb{R}_{+}$of the Log-approximated density is, by construction, equal to one (cf. Remark 2.5)

$$
\int_{0}^{+\infty} \Gamma_{\log }\left(0, S_{0} ; T, y\right) d y=1
$$

for any positive $T$ and $S_{0}$. This is not the case for the Gaussian approximation by Hagan-Woodward that verifies

$$
\int_{0}^{+\infty} \Gamma_{\mathrm{HW}}\left(0, S_{0} ; T, y\right) d y \leq 1 .
$$



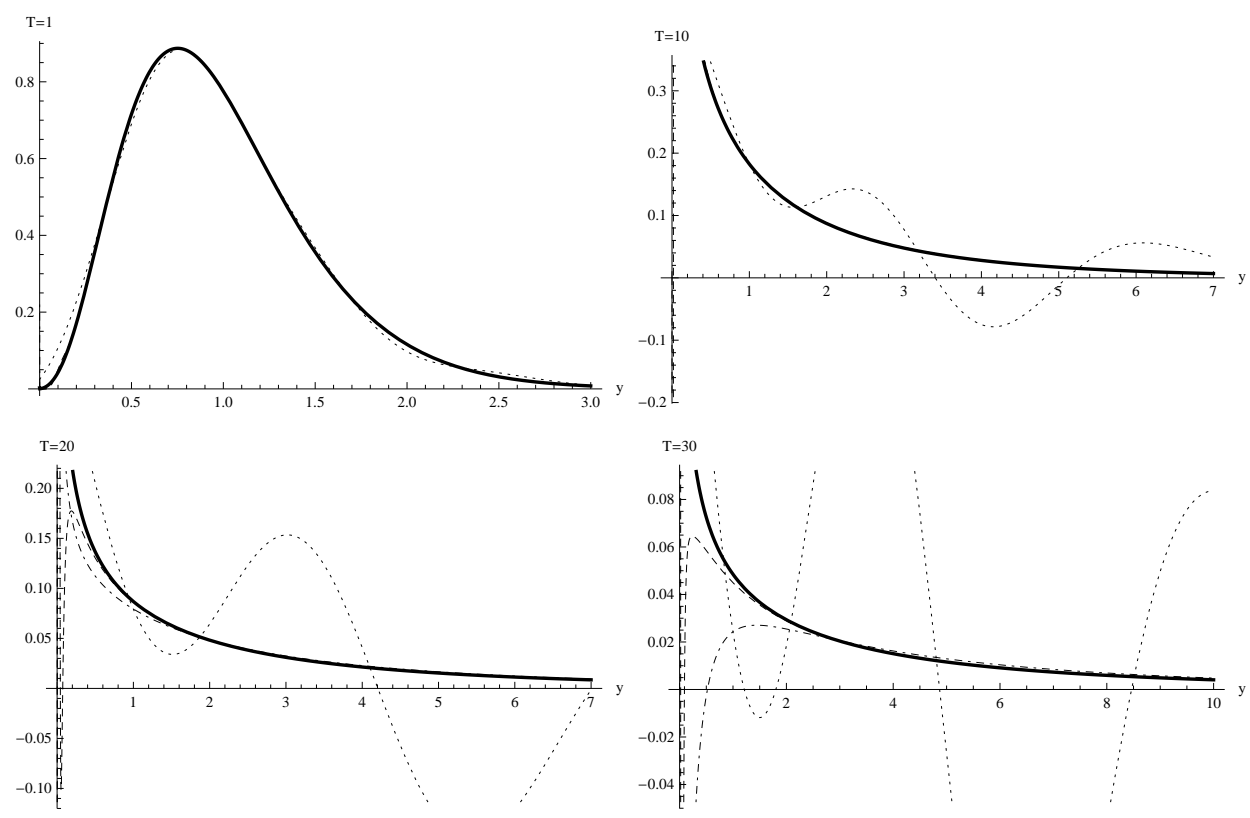

Figure 4: CEV density with $\beta=\frac{2}{3}$ (thick line), HL approximation (dot-dashed line), HW approximation (dotted line) and 4th-order Log-approximation (dashed line) with parameters $S_{0}=1, \sigma=0.5, r=0$ and maturities $T=1, T=10, T=20$ and $T=30$
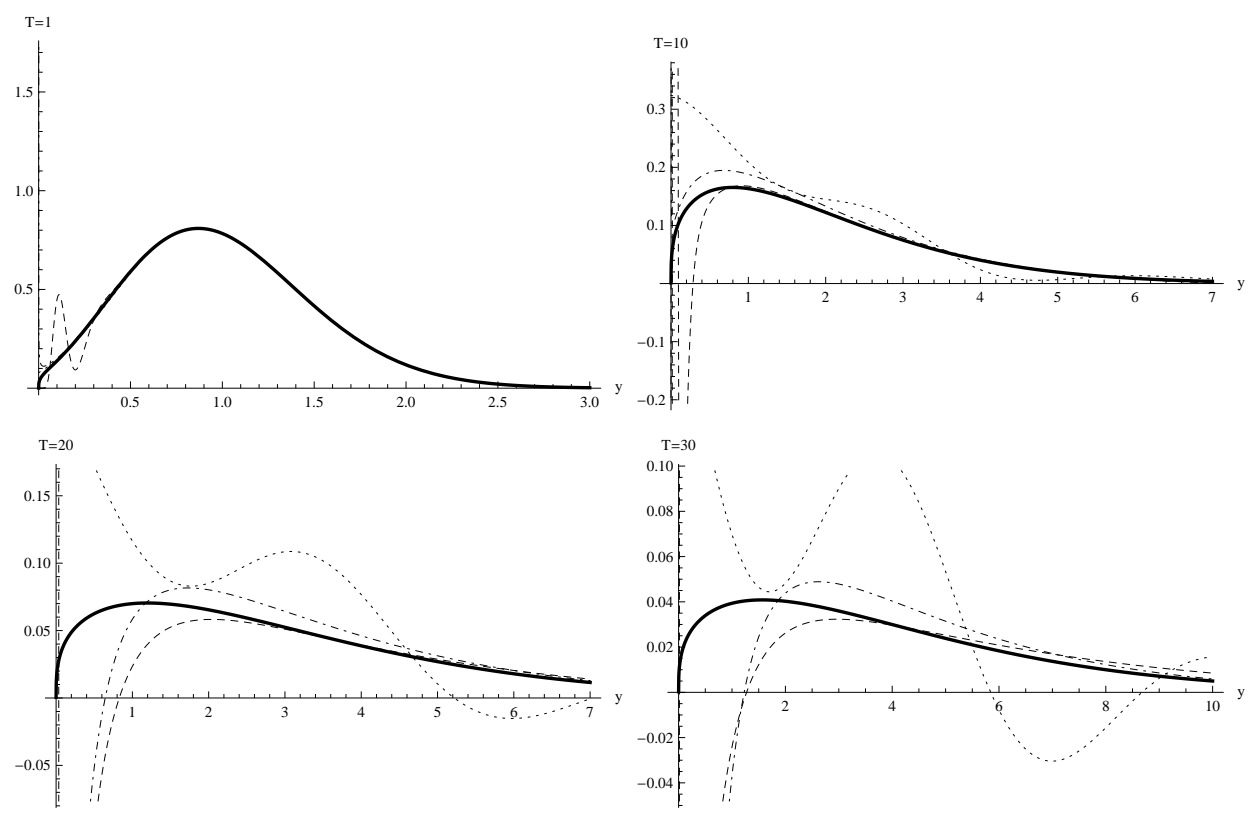

Figure 5: CEV density with $\beta=\frac{1}{3}$ (thick line), HL approximation (dot-dashed line), HW approximation (dotted line) and 4th-order Log-approximation (dashed line) with parameters $S_{0}=1, \sigma=0.5, r=0$ and maturities $T=1, T=10, T=20$ and $T=30$ 


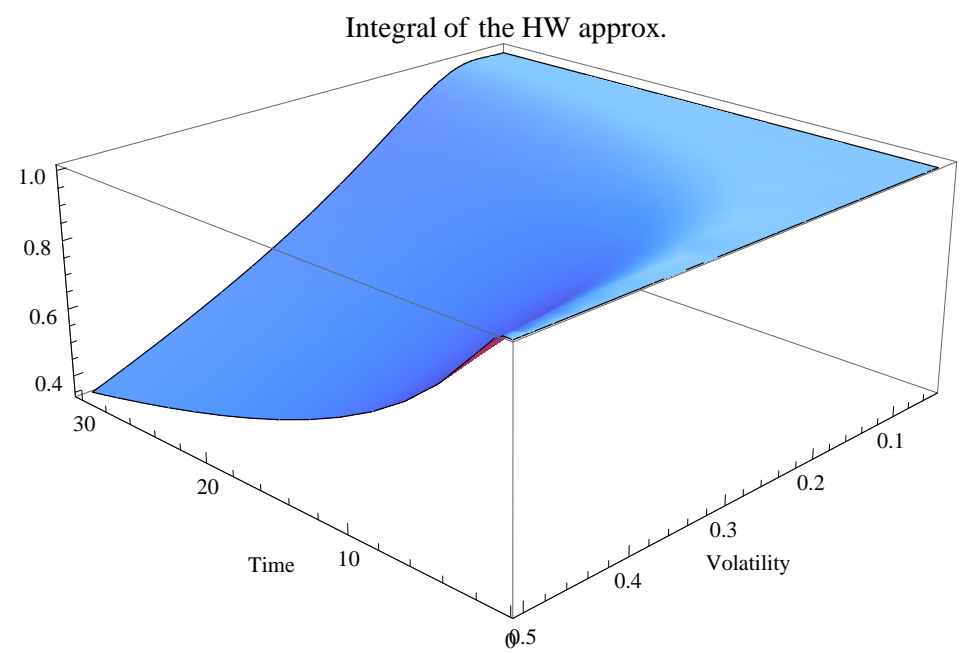

Figure 6: Integral of the HW density as a function of the volatility $\sigma \in[5 \%, 50 \%]$ and maturity $T \in\left[\frac{1}{2}, 30\right]$ in the CEV model with $\beta=\frac{1}{2}$

In Figure 6 the value of the integral in (4.43), expressed as a function of the volatility $\sigma \in[5 \%, 50 \%]$ and maturity $T \in\left[\frac{1}{2}, 30\right]$, is plotted; the other parameters are $\beta=\frac{1}{2}, r=0$ and $S_{0}=1$. Also the integral of the LH density is generally different from one, as shown in Figure 7.

Call and Put prices $(\times 10)$

\begin{tabular}{llllllll}
\hline & Bessel & 4th-Log & 2nd-Log & HW Call & HW Put & LH Call & LH Put \\
\hline$T=1$ & 1.19345 & 1.19345 & 1.19344 & 1.19346 & 1.19174 & 1.19359 & 1.1939 \\
$T=5$ & 2.63769 & 2.63768 & 2.63737 & 2.63855 & 2.40181 & 2.64278 & 2.70363 \\
$T=10$ & 3.67286 & 3.67295 & 3.67201 & 3.67825 & 2.39639 & 3.69572 & 3.53664 \\
$T=20$ & 5.01275 & 5.01915 & 5.02073 & 5.0513 & 1.97434 & 5.11899 & 3.14135 \\
$T=30$ & 5.89194 & 5.91281 & 5.92962 & 6.00219 & 1.64068 & 6.14735 & 1.87179 \\
\hline
\end{tabular}

Table 1: ATM Call and Put options in the CEV model with $\beta=\frac{1}{2}, \sigma=30 \%$ and $r=0$ computed by convolution with the "Bessel" density in (4.40) (second column), the 4-th order Log-approximated density (third column), the 2nd order Log-approximated density (fourth column), the Hagan-Woodward approximated density (fifth and sixth columns) and the Henry-Labordère approximated density (seventh and eighth columns)

This fact is noteworthy because when the integral in (4.43) is different from one, then option prices produced by the approximated density $\Gamma_{\mathrm{HW}}$ give rise to arbitrage opportunities. Indeed let us recall that, according to the Put-Call parity, when $r=0$ the prices of at-the-money (ATM) Call and Put options must coincide. In Table 1 , for the set of parameters $\beta=\frac{1}{2}$, 


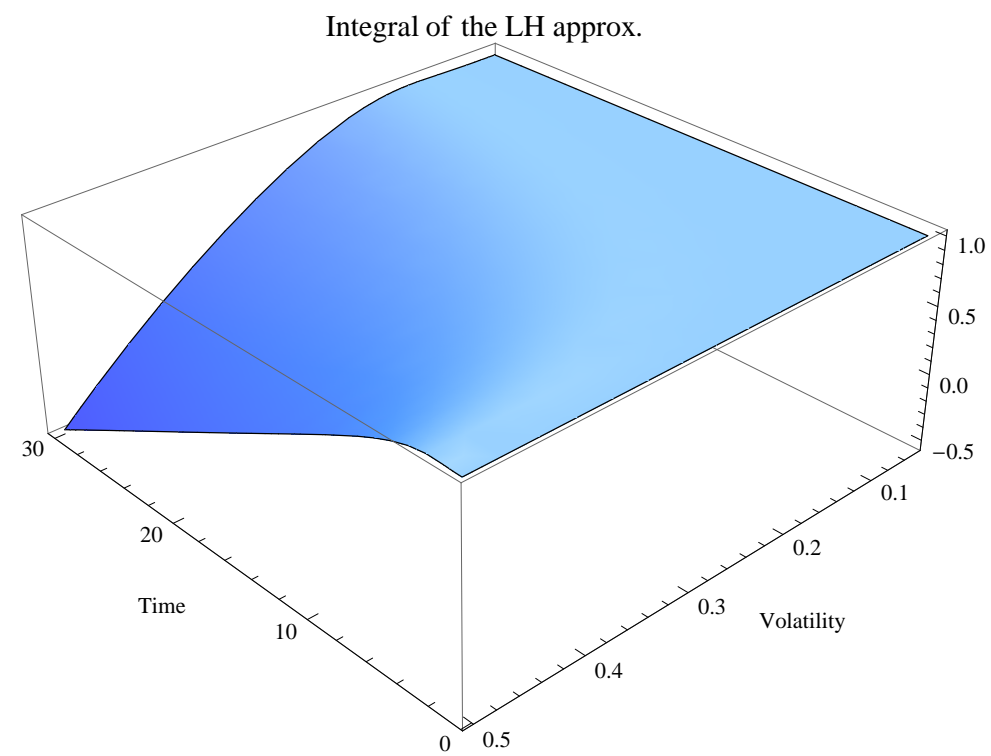

Figure 7: Integral of the LH density as a function of the volatility of the volatility $\sigma \in[5 \%, 50 \%]$ and maturity $T \in\left[\frac{1}{2}, 30\right]$ in the CEV model with $\beta=\frac{1}{2}$

$\sigma=30 \%, r=0, K=S_{0}=1$, we report the numerical results obtained by direct integration of the payoff functions of Call and Put options, convoluted with the "Bessel" density in (4.40) (second column), with the 4-th order Logapproximated density (third column), with the 2-nd order Log-approximated density (fourth column), with the Hagan-Woodward approximated density (fifth and sixth columns) and with the Henry-Labordère approximated density (seventh and eighth columns) respectively. In Table 2 we report the analogous results in the case $\beta=\frac{1}{10}$.

For the Bessel and Log-approximated densities, Puts and Calls have indeed the same price and in the table we report the common value; on the contrary, Put prices in the HW and LH approximations become uncorrect for long maturities and violate the Put-Call parity formula.

We recall that Hagan and Woodward give in [18] a well-known approximation formula of the implied volatility: the formula is rather satisfactory because it is obtained from the approximation of Call prices that are not affected by the singularity of the density at the origin. However, as the previous analysis shows, the HW approximation is less satisfactory from the more general point of view of the estimate of the density function, especially for pricing purposes.

We conclude this section by showing a comparison between the HW and Log approximation formulae of Call options: we take as reference values, the prices computed using the exact density in (4.40) (cf. Shaw [29]) with parameters $r=5 \%$ and $S_{0}=1$. For each test we consider the strike ranging 
Call and Put prices $(\times 10)$

\begin{tabular}{llllllll}
\hline & Bessel & 4th-Log & 2nd-Log & HW Call & HW Put & LH Call & LH Put \\
\hline$T=1$ & 1.19596 & 1.19595 & 1.19587 & 1.19597 & 1.19418 & 1.19601 & 1.19531 \\
$T=5$ & 2.66435 & 2.66417 & 2.66094 & 2.66665 & 1.91262 & 2.66744 & 1.97186 \\
$T=10$ & 3.71811 & 3.73689 & 3.72705 & 3.75773 & 1.74907 & 3.76049 & 1.75563 \\
$T=20$ & 4.97979 & 5.10287 & 5.11945 & 5.2761 & 1.4027 & 5.28507 & 1.16869 \\
$T=30$ & 5.72782 & 5.84894 & 6.02539 & 6.41517 & 1.18081 & 6.43251 & 0.63639 \\
\hline
\end{tabular}

Table 2: ATM Call and Put options in the CEV model with $\beta=\frac{1}{10}, \sigma=30 \%$ and $r=0$ computed by convolution with the "Bessel" density in (4.40) (second column), the 4-th order Log-approximated density (third column), the 2nd order Log-approximated density (fourth column), the Hagan-Woodward approximated density (fifth and sixth columns) and the Henry-Labordère approximated density (seventh and eighth columns)

from $\frac{1}{2}$ to 2 , maturities from $\frac{1}{10}$ to 30 years and typical values of the volatility $\sigma=30 \%$ and $\sigma=80 \%$. We first consider maturities $T \leq 5$ : in Figures 8 and 9 we plot the relative errors for $\beta=\frac{2}{3}$ and $\beta=\frac{1}{3}$ respectively. Figures 10 and 9 are analogous but for longer maturities, up to 30 years.

\section{References}

[1] F. Antonelli and S. Scarlatti, Pricing options under stochastic volatility: a power series approach, Finance Stoch., 13 (2009), pp. 269303.

[2] J. P. Barjaktarevic and R. Rebonato, Approximate solutions for the SABR model: Improving on the Hagan expansion, Talk at ICBI Global Derivatives Trading and Risk Management Conference, (2010).

[3] E. Benhamou, E. Gobet, And M. Miri, Expansion formulas for European options in a local volatility model, Int. J. Theor. Appl. Finance, 13 (2010), pp. 603-634.

[4] H. Berestycki, J. Busca, And I. Florent, Computing the implied volatility in stochastic volatility models, Comm. Pure Appl. Math., 57 (2004), pp. 1352-1373.

[5] L. CAPRIOTti, The exponent expansion: an effective approximation of transition probabilities of diffusion processes and pricing kernels of financial derivatives, Int. J. Theor. Appl. Finance, 9 (2006), pp. 11791199. 


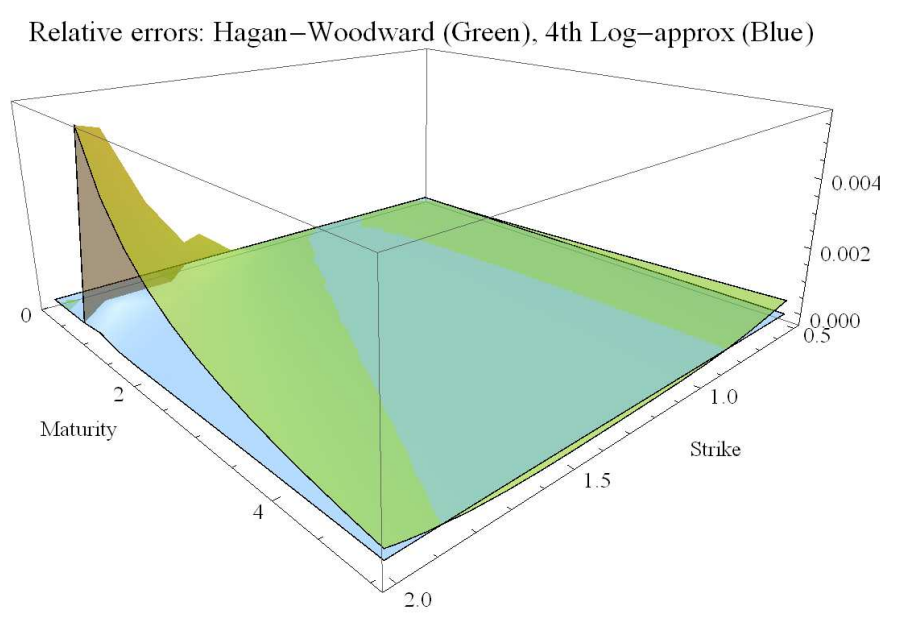

Relative errors: Hagan-Woodward (Green), 4th Log-approx (Blue)

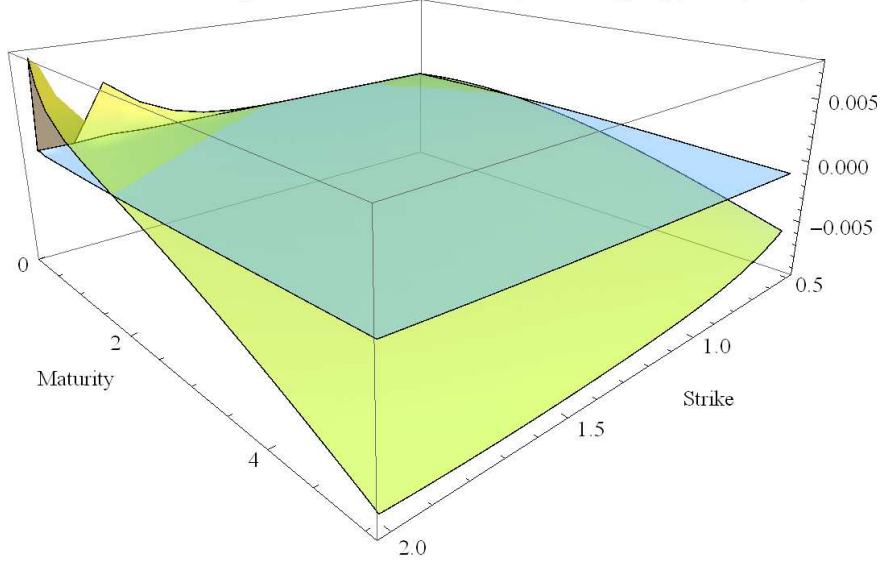

Figure 8: Relative errors for Call prices in the HW approximation (green) and 4th Log-approximation (blue) for $\beta=\frac{2}{3}$, maturities $T \in\left[\frac{1}{10}, 5\right]$ and $\sigma=30 \%$ (top) and $\sigma=80 \%$ (bottom)

[6] W. Cheng, R. Constantinescu, N. Costanzino, A. Mazzucato, AND V. NisToR, Closed form asymptotics for stochastic volatility models, Working paper, (2011).

[7] F. Corielli, P. Foschi, And A. Pascucci, Parametrix approximation of diffusion transition densities, SIAM J. Financial Math., 1 (2010), pp. 833-867.

[8] J. C. Cox, Notes on option pricing I: constant elasticity of variance diffusion, Working paper, Stanford University, Stanford CA, (1975).

[9] D. Davydov and V. Linetsky, Pricing and hedging path-dependent options under the CEV process, Management Science, 47 (2001), pp. 949-965. 


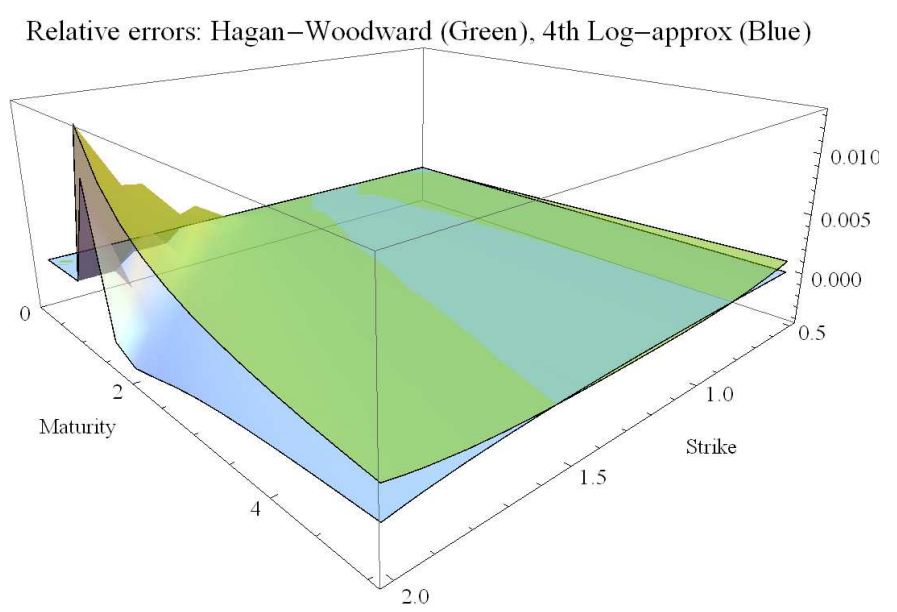

Relative errors: Hagan-Woodward (Green), 4th Log-approx (Blue)

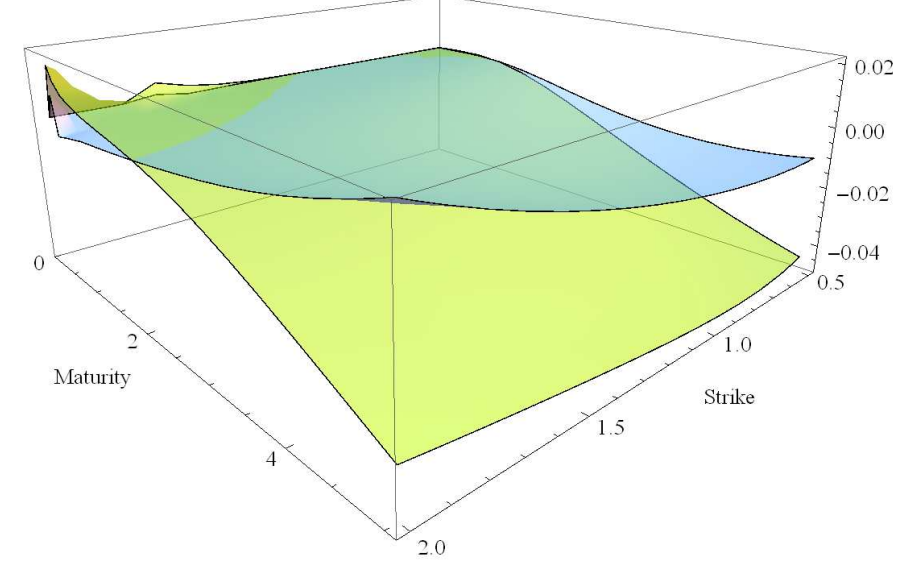

Figure 9: Relative errors for Call prices in the HW approximation (green) and 4th Log-approximation (blue) for $\beta=\frac{1}{3}$, maturities $T \in\left[\frac{1}{10}, 5\right]$ and $\sigma=30 \%$ (top) and $\sigma=80 \%$ (bottom)

[10] F. Delbaen and H. Shirakawa, A note on option pricing for the constant elasticity of variance model, Asia-Pacific Financial Markets, 9 (2002), pp. 85-99. 10.1023/A:1022269617674.

[11] P. Doust, No arbitrage SABR, working paper, (2010).

[12] E. Ekström AND J. Tysk, Boundary behaviour of densities for nonnegative diffusions, preprint, (2011).

[13] P. Foschi, S. Pagliarani, and A. Pascucci, Black-Scholes formulae for Asian options, Working paper, (2011).

[14] J.-P. Fouque, G. Papanicolaou, R. Sircar, and K. Solna, Singular perturbations in option pricing, SIAM J. Appl. Math., 63 (2003), pp. 1648-1665 (electronic). 


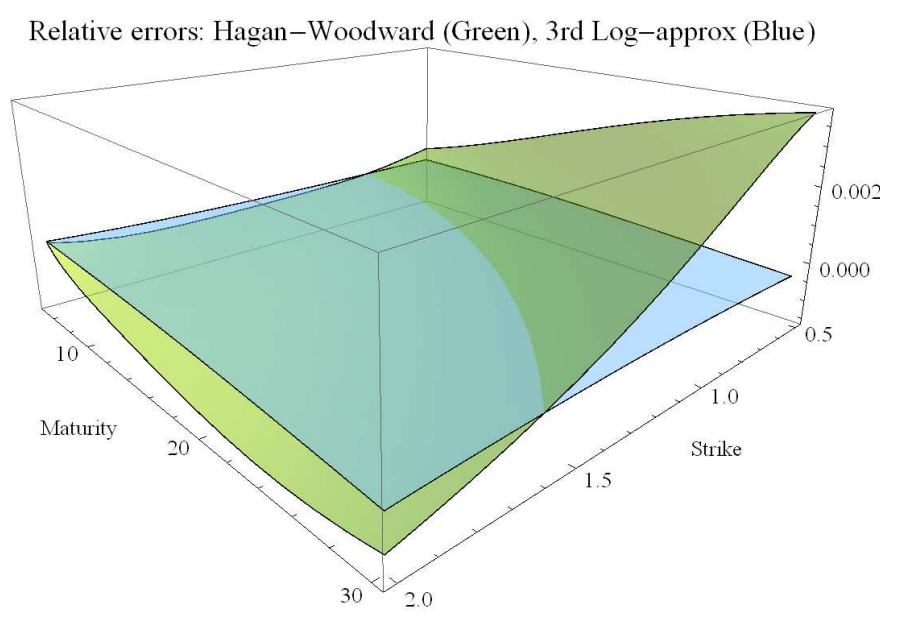

Relative errors: Hagan-Woodward (Green), 3rd Log-approx (Blue)

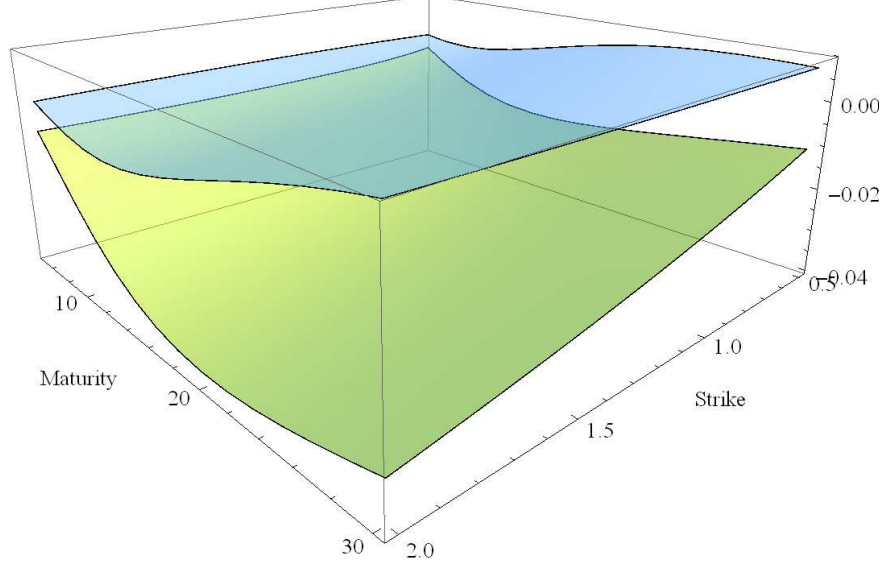

Figure 10: Relative errors for Call prices in the HW approximation (green) and 3rd Log-approximation (blue) for $\beta=\frac{2}{3}$, maturities $T \in[5,30]$ and $\sigma=30 \%$ (top) and $\sigma=80 \%$ (bottom)

[15] J. Gatheral, E. P. Hsu, P. Laurence, C. Ouyang, and T.-H. WANG, Asymptotics of implied volatility in local volatility models, to appear in Math. Finance, (2010).

[16] P. Hagan, D. Kumar, A. Lesniewski, and D. Woodward, Managing smile risk, Wilmott, September (2002), pp. 84-108.

[17] P. Hagan, A. Lesniewski, And D. Woodward, Managing smile risk, Wilmott, September (2002), pp. 84-108.

[18] P. Hagan and D. Woodward, Equivalent Black volatilities, Appl. Math. Finance, 6 (1999), pp. 147-159. 


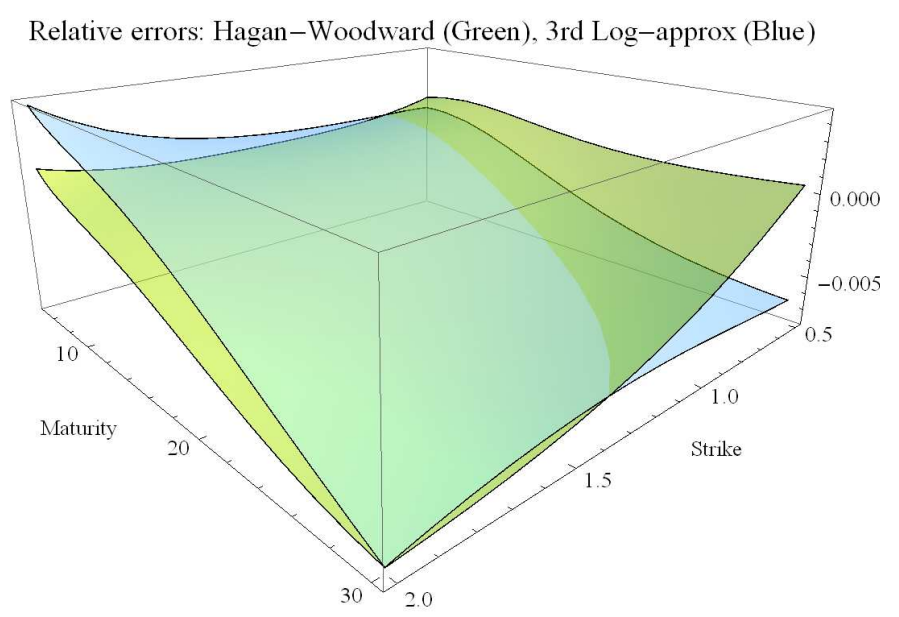

Relative errors: Hagan-Woodward (Green), 3rd Log-approx (Blue)

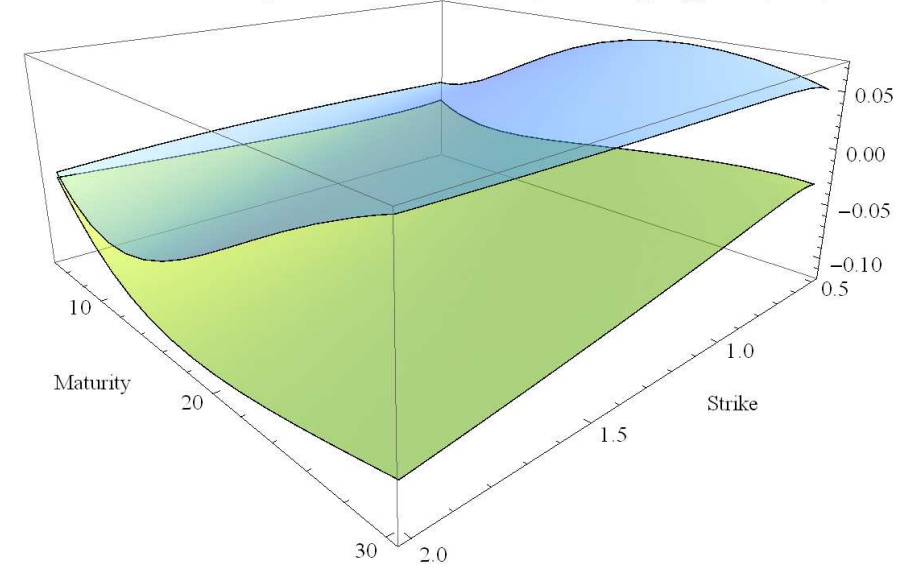

Figure 11: Relative errors for Call prices in the HW approximation (green) and 3rd Log-approximation (blue) for $\beta=\frac{1}{3}$, maturities $T \in[5,30]$ and $\sigma=30 \%$ (top) and $\sigma=80 \%$ (bottom)

[19] P. Henry-Labordère, A general asymptotic implied volatility for stochastic volatility models, Frontiers in Quantitative Finance, Wiley, (2008).

[20] — Analysis, geometry, and modeling in finance, Chapman \& Hall/CRC Financial Mathematics Series, CRC Press, Boca Raton, FL, 2009. Advanced methods in option pricing.

[21] S. L. Heston, M. Loewenstein, and G. A. Willard, Options and Bubbles, The Review of Financial Studies, Vol. 20, Issue 2, pp. 359-390, (2007).

[22] S. Howison, Matched asymptotic expansions in financial engineering, J. Engrg. Math., 53 (2005), pp. 385-406. 
[23] S. JAnson And J. Tysk, Feynman-Kac formulas for Black-Scholestype operators, Bull. London Math. Soc., 38 (2006), pp. 269-282.

[24] D. Kristensen And A. Mele, Adding and subtracting Black-Scholes: A new approach to approximating derivative prices in continuous time models, to appear in Journal of Financial Economics, (2011).

[25] A. LeSniewski, Swaption smiles via the WKB method, Mathematical Finance Seminar, Courant Institute of Mathematical Sciences, (2002).

[26] A. Lindsay and D. Brecher, Results on the CEV Process, Past and Present, SSRN eLibrary, (2010).

[27] A. PASCUCCI, PDE and martingale methods in option pricing, Bocconi\&Springer Series, Springer-Verlag, New York, 2011.

[28] L. Paulot, Asymptotic Implied Volatility at the Second Order with Application to the SABR Model, SSRN eLibrary, (2009).

[29] W. T. SHaw, Modelling financial derivatives with Mathematica, Cambridge University Press, Cambridge, 1998. Mathematical models and benchmark algorithms, With 1 CD-ROM (Windows, Macintosh and UNIX).

[30] S. TAYLOR, Perturbation and symmetry techniques applied to finance, Ph. D. thesis, Frankfurt School of Finance \& Management. Bankakademie HfB, (2011).

[31] A. E. Whalley And P. Wilmott, An asymptotic analysis of an optimal hedging model for option pricing with transaction costs, Math. Finance, 7 (1997), pp. 307-324.

[32] M. Widdicks, P. W. Duck, A. D. Andricopoulos, And D. P. Newton, The Black-Scholes equation revisited: asymptotic expansions and singular perturbations, Math. Finance, 15 (2005), pp. 373-391. 


\title{
Analytical approximation of transition densities in option pricing: local volatility models. Version 1.0
}

\author{
Stefano Pagliarani \\ Dipartimento di Matematica \\ Università di Padova \\ Via Trieste 63, \\ 35121 Padova, Italy \\ stefanop@math.unipd.it \\ Andrea Pascucci \\ Dipartimento di Matematica \\ Università di Bologna \\ Piazza di Porta san Donato, 5 \\ Bologna, Italy \\ andrea.pascucci@unibo.it
}

\begin{abstract}
This notebook contains the implementation of analytical approximation formulae for the transition density and the Call prices, up to the 4th order, in a general local volatility model of the form

$$
d S_{t}=r S_{t} d t+\sigma\left(S_{t}\right) S_{t} d W_{t} .
$$
\end{abstract}

The method is sufficiently general to be extended to time-dependent coefficients, multidimensional stochastic volatility models (e.g. the SABR model), degenerate parabolic pdes related to Asian options and also to include jumps.

24 April 2011

\section{Disclaimer}

This Mathematica notebook is provided on an "as is" basis without warranty of any kind, express or implied. Under no circumstances and under no legal theory, whether in tort, contract, or otherwise, shall the authors be liable to you or to any other person for any indirect, special, incidental, or consequential damages or losses. If you do not agree with these terms, then you you are advised to not use the notebook. If you wish to endorse this notebook, then please link to the webpage of the authors.

\section{Introduction}

This notebook implements the formulae given in the paper

[PP2011] S. Pagliarani, A. Pascucci, “Closed form densities for local volatilty models,” preprint AMS Acta, 2011 pp. 1-27,

for the approximation of the transition density and Call price in a generic local volatilty model. In the sequel we systematically refer to that paper for the notations and the main results: in particular we refer to the labelled formulae in [PP2011] as to (PP- $n$ ) where $n$ is the number of the formula.

The method proposed in [PP2011] is sufficiently general to be extended to time-dependent coefficients, multi-dimensional stochastic volatility models, degenerate parabolic pdes related to Asian options and also to include jumps: we are currently working on the implementation of these generalizations that will appear in the future releases of this notebook.

\section{General notations}

We consider the following risk-neutral dynamics in the local volatility (LV) model

$$
d S_{t}=r S_{t} \mathrm{dt}+\sigma\left(S_{t}\right) S_{t} d W_{t} .
$$


2 | explicit solutions.nb

The related pricing Cauchy problem is

$$
\mathbf{L} C(t, S)=0, \quad t<T, \quad S>0
$$

with

$$
C(T, S)=\varphi(S), \quad S>0,
$$

where

$$
\mathbf{L}=\frac{1}{2} \sigma^{2}(s) s^{2} \partial_{s, s}+r s \partial_{s}+\partial_{t}-r .
$$

When $\sigma$ is uniformly positive, it is more effective to approximate the operator after the log-change of variables

$$
u(t, x)=e^{r(T-t)} C\left(t, e^{x}\right) .
$$

Thus we have the equation

$$
L u(t, x)=0, \quad t<T, x \in \mathbb{R}
$$

with final condition

$$
u(T, x)=\varphi\left(e^{x}\right), \quad x \in \mathbb{R},
$$

for the parabolic operator

$$
L=\frac{1}{2} a(x)\left(\partial_{x, x}-\partial_{x}\right)+r \partial_{x}+\partial_{t}, \quad a(x)=\sigma^{2}\left(e^{x}\right) .
$$

If $P$ and $\Gamma$ denote the fundamental solutions of $\mathbf{L}$ and $L$ respectively, then we have

$$
P\left(t, S_{0} ; T, S\right)=\frac{1}{S} \Gamma\left(t, \log S_{0} ; T, \log S\right) .
$$

Using the Taylor expansion of $a(x)$ with starting point $\bar{x}$ we get

$$
\frac{1}{2} a(x)=\frac{1}{2} \alpha_{0}+\sum_{n=1}^{+\infty} \alpha_{n}(x-\bar{x})^{n}
$$

where

$$
\alpha_{0}=a(\bar{x}), \quad \alpha_{n}=\frac{1}{2 n !} \frac{d^{n}}{\mathrm{dx}^{n}} a(\bar{x}), \quad n \geq 1 .
$$

Thus L can be formally rewritten as

$$
L=L^{0}+\sum_{n=1}^{+\infty} \alpha_{n}(x-\bar{x})^{n}\left(\partial_{x, x}-\partial_{x}\right)
$$

where

$$
L^{0}=\frac{1}{2} \alpha_{0}\left(\partial_{x, x}-\partial_{x}\right)+r \partial_{x}+\partial_{t}
$$

\section{Approximation methodology}

We compute the first and second order approximations of the density $\Gamma$ of operator L. In general, according to [PP2011], the $N$-th order approximation of $\Gamma$ is of the form

$$
\Gamma(t, x ; T, z) \approx \Gamma^{N}(t, x ; T, z)=\sum_{n=0}^{N} G^{n}(t, x ; T, z)
$$

where $G^{0}=\Gamma^{0}$ is the fundamental solution of of $L^{0}$ 


$$
\Gamma[0]\left[t_{-}, x_{-}, T_{-}, z_{-}, r_{-}, \alpha 0_{-}\right]:=\frac{e^{-\frac{\left(x-z+(-t+T)\left(x-\frac{\alpha 0}{2}\right)\right)^{2}}{2(-t+T) \alpha 0}}}{\sqrt{2 \pi} \sqrt{(-t+T) \alpha 0}} ;
$$

and

$$
G^{n}(t, x ; T, z)=J^{n}(t, T, x) \Gamma^{0}(t, x ; T, z)
$$

for some differential operator $J^{n}(t, T, x)$ of the form

$$
J^{n}(t, T, x)=\sum_{i=1}^{3 n} \sum_{j=0}^{n} f_{i, j}^{n}(t, T, x)(x-\bar{x})^{j} \frac{\partial^{i}}{\partial x^{i}} .
$$

We proceed as in the proof Proposition PP-2.3 to find the explicit expression of the coefficients $f_{i, j}^{n}$ for $n=1,2$. To this end we first define the differentiation and product operators that are analogous, in our more general setting, to the operators introduced in Lemma PP-2.2:

$$
\begin{aligned}
& \mathrm{w}\left[\mathrm{x}_{-}, \mathrm{u}_{-}\right]:=\partial_{\mathbf{x}} \mathrm{u}_{;} \\
& \mathrm{v}\left[\mathrm{t}_{-}, \mathrm{T}_{-}, \mathrm{x}_{-}, \mathrm{r}_{-}, \mathrm{xbar}_{-}, \alpha 0_{-}, \mathrm{u}_{-}\right]:= \\
& \quad\left(\mathrm{x}+\left(\mathrm{r}-\frac{\alpha 0}{2}\right)(\mathrm{T}-\mathrm{t})-\mathrm{xbar}\right) \mathrm{u}+\alpha 0(\mathrm{~T}-\mathrm{t}) \partial_{\mathbf{x}} \mathrm{u}
\end{aligned}
$$

Next we recall that

$$
G^{1}(t, x ; T, z)=J^{1}(t, T, x) \Gamma^{0}(t, x ; T, z)=\int_{t}^{T} \int_{-\infty}^{+\infty} \Gamma^{0}(t, x ; s, \eta) \alpha_{1}(\eta-\bar{x}) \Gamma^{0}(s, \eta ; T, z) d \eta d s
$$

and therefore, setting $\mathrm{F}[\mathrm{x}]=\Gamma^{0}(t, x ; T, z)$, we have

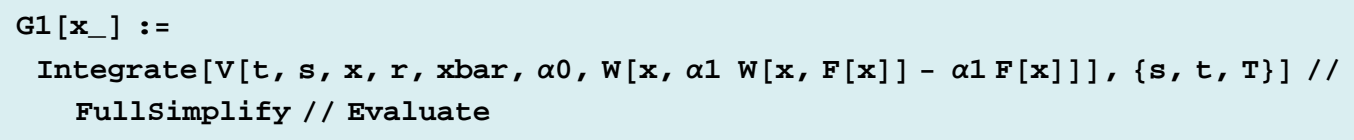

Now we give the expression of the coefficients $f_{i, j}^{n}$ for $n=1$ and define operator $\mathrm{J} 1$ as in (3):

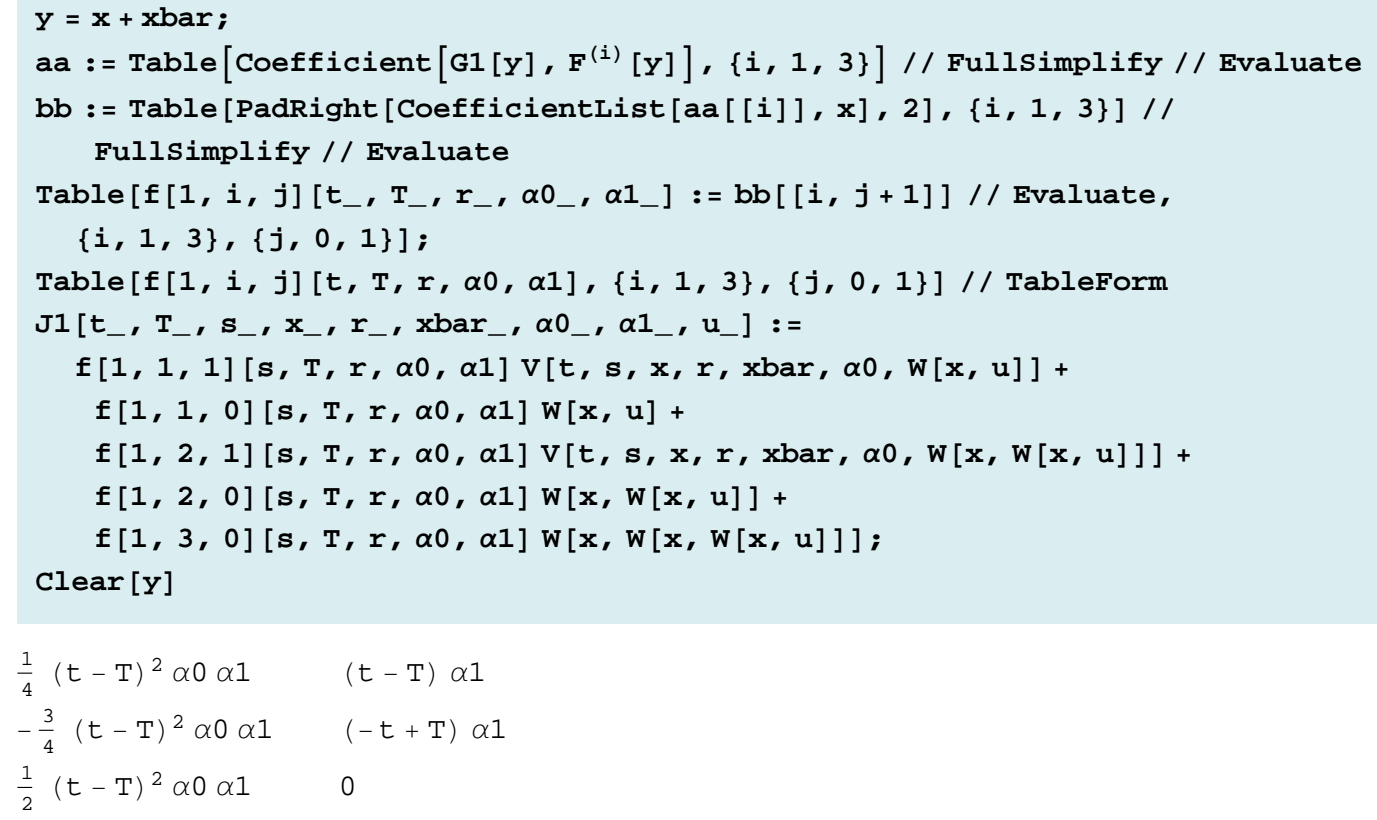

Concerning $G^{2}$, we have 


$$
\begin{gathered}
G^{2}(t, x ; T, z)= \\
J^{2}(t, T, x) \Gamma^{0}(t, x ; T, z)=\int_{t}^{T} \int_{-\infty}^{+\infty} \Gamma^{0}(t, x ; s, \eta)\left(\alpha_{1}(\eta-\bar{x}) G^{1}(s, \eta ; T, z)+\alpha_{2}(\eta-\bar{x})^{2} \Gamma^{0}(s, \eta ; T, z)\right) d \eta d s ;
\end{gathered}
$$

In terms of the operators $V$ and $W, G^{2}$ takes the form (cf. (PP-2.23))

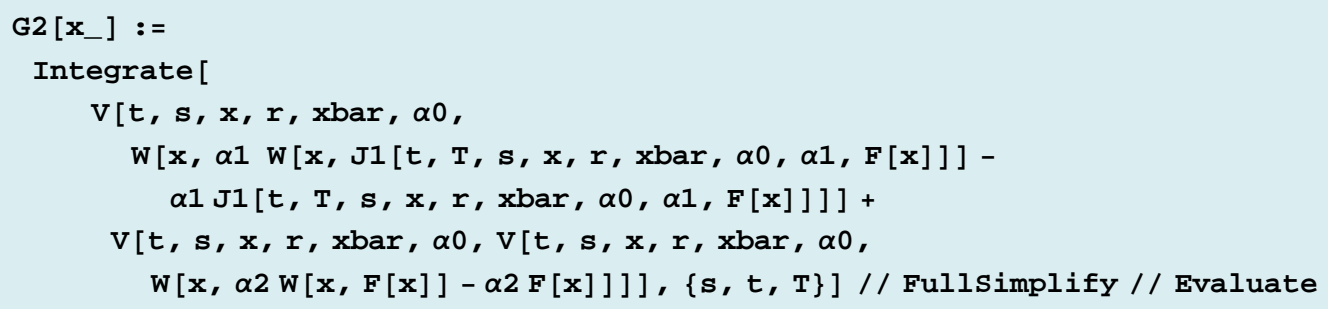

and the expression of the coefficients $f_{i, j}^{n}$ for $n=2$ is as follows

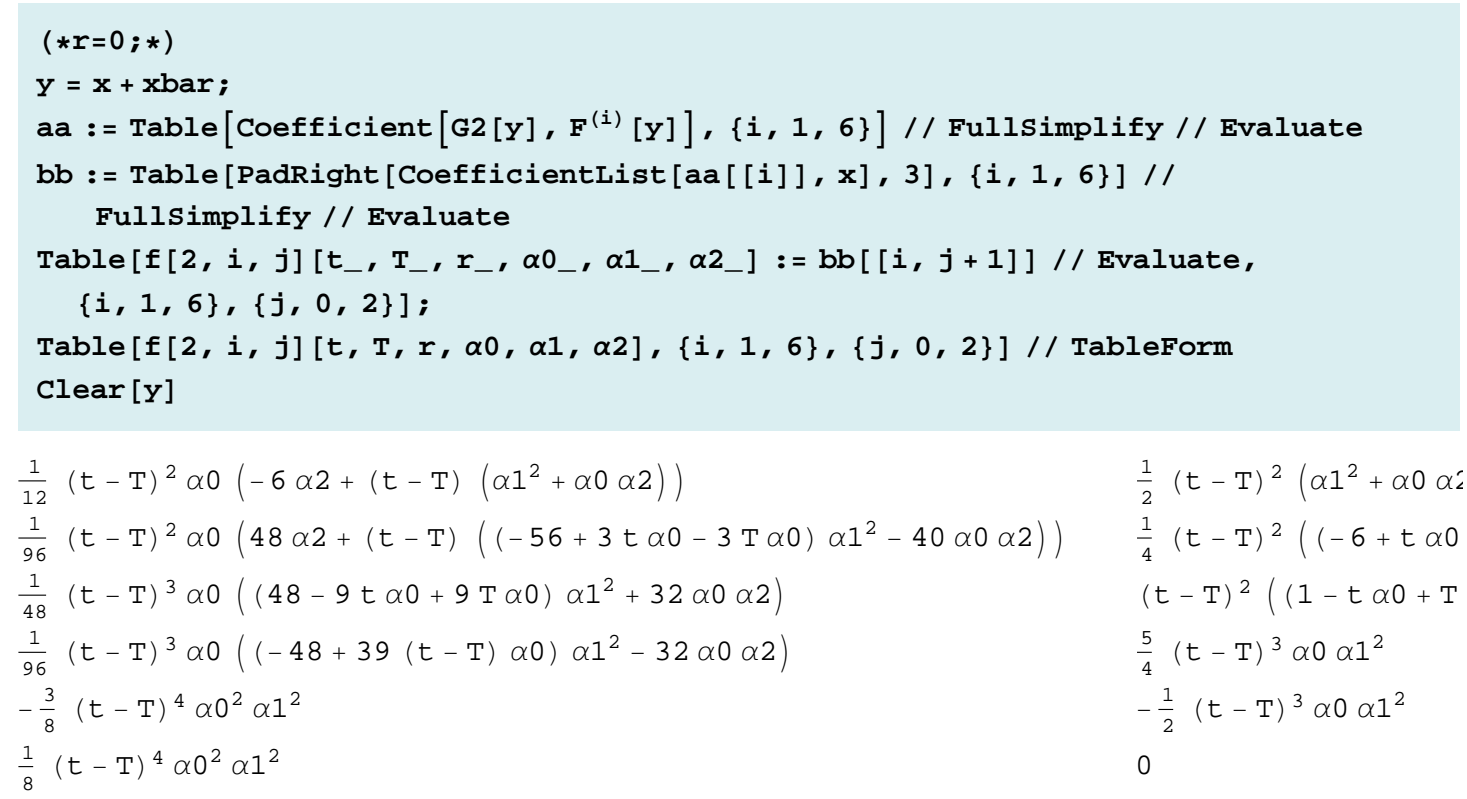

\section{Approximation of the density}

From now on we consider the particular case $\bar{x}=x$. Then we have

$$
G^{n}(t, x ; T, z)=\sum_{i=1}^{3 n} f_{i, 0}^{n}(t, T, x) \frac{\partial^{i}}{\partial x^{i}} \Gamma^{0}(t, x ; T, z) .
$$

We recall that the derivatives of $\Gamma^{0}$ can be written in terms of the Hermite polynomials as follows:

$$
\frac{\frac{\partial^{i}}{\partial x^{i}} \Gamma^{0}(t, x ; T, z)}{\Gamma^{0}(t, x ; T, z)}=(-1)^{i} \frac{1}{\left(\sqrt{2(T-t) \alpha_{0}}\right)^{i}} H\left(i, \frac{x-z+(T-t)\left(r-\frac{\alpha_{0}}{2}\right)}{\sqrt{2(T-t) \alpha_{0}}}\right)
$$

and therefore

$$
G^{n}(t, x ; T, z)=\mathrm{GG}^{n}(t, x ; T, z) \Gamma^{0}(t, x ; T, z)
$$

where

$$
\mathrm{GG}^{n}(t, x ; T, z)=\sum_{i=1}^{3 n} f_{i, 0}^{n}(t, T, x)(-1)^{i} \frac{1}{\left(\sqrt{2(T-t) \alpha_{0}}\right)^{i}} H\left(i, \frac{x-z+(T-t)\left(r-\frac{\alpha_{0}}{2}\right)}{\sqrt{2(T-t) \alpha_{0}}}\right)
$$

In particular, for $n=1,2$, we set 


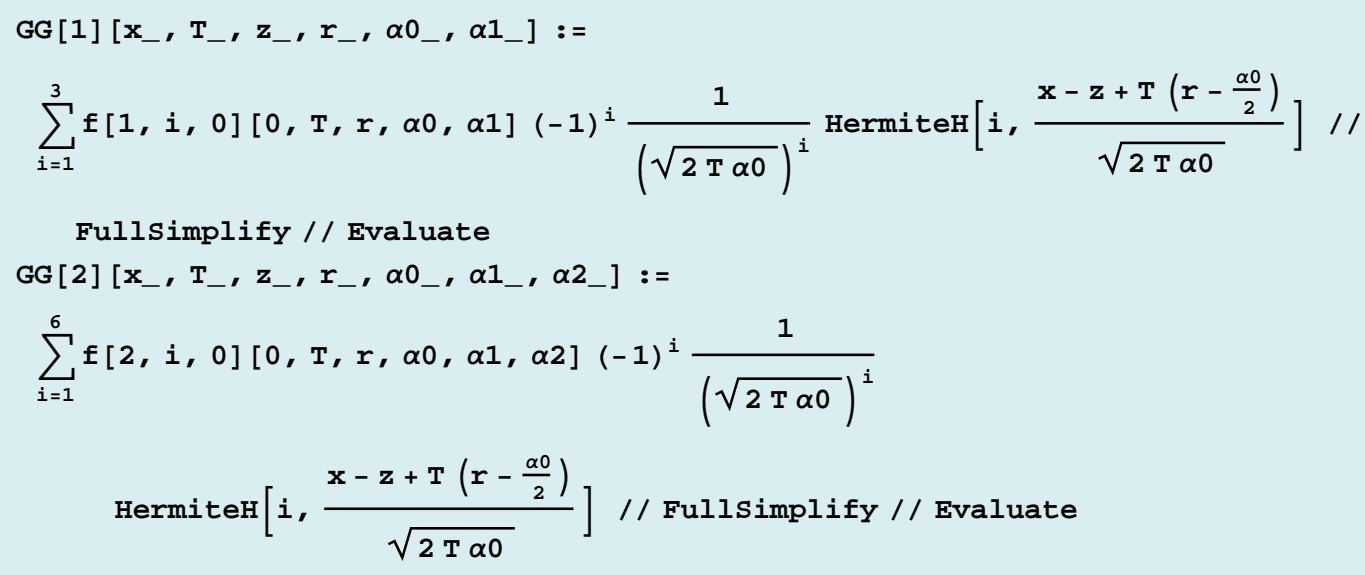

Hence the approximations $\Gamma^{1}$ and $\Gamma^{2}$ are given by

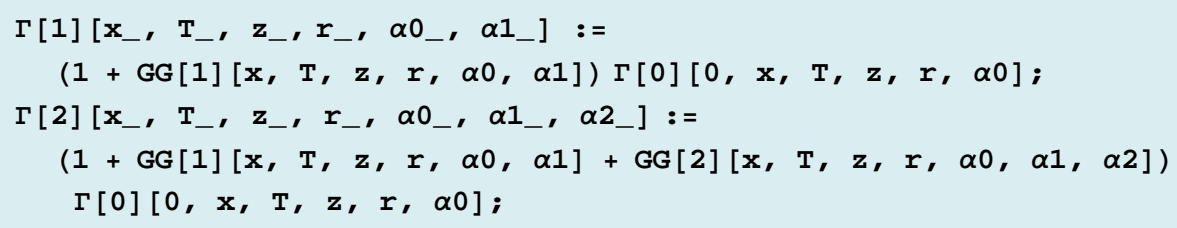

moreover we denote by $Г \Gamma[i], i=1,2$, the first and second order approximations of the density of the operator $\mathbf{L}$ in (2):

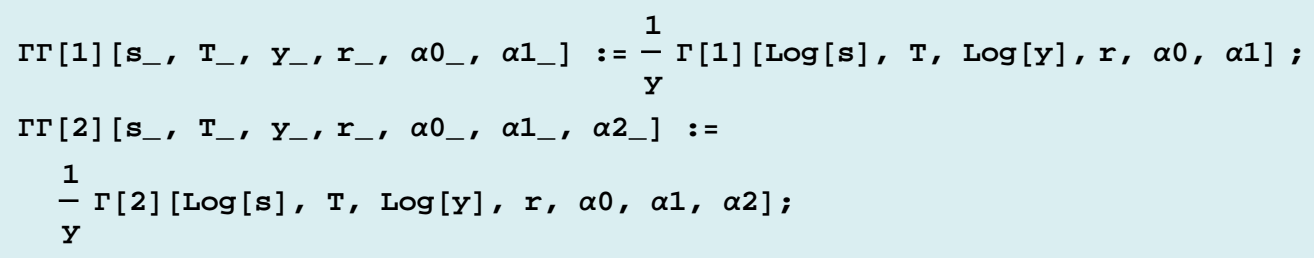

\section{Approximation of the Call price}

The $N$-th order approximation of the Call price $\mathrm{C}\left(0, S_{0}\right)$ is given by

$$
e^{-r T} u^{N}\left(0, \log S_{0}\right)
$$

where

$$
u^{N}(t, x)=C_{\mathrm{BS}}(t, x)+\sum_{n=1}^{N} J^{n}(t, T, x) C_{\mathrm{BS}}(t, x)
$$

and $C_{\mathrm{BS}}(\mathrm{t}, \mathrm{x})$ is standard Black \& Scholes function (of the log-price):

$$
C_{\mathrm{BS}}(t, x)=\int_{-\infty}^{+\infty} \frac{1}{y} \Gamma^{0}(t, x ; T, \log y)(y-K)^{+} d y .
$$

In order to find the first and second order approximations, we just need to compute $C_{\mathrm{BS}}(0, \mathrm{x})$ and its derivatives: since the derivatives of $C_{\mathrm{BS}}(0, \mathrm{x})$ contain a common additive term, we compute it separately 


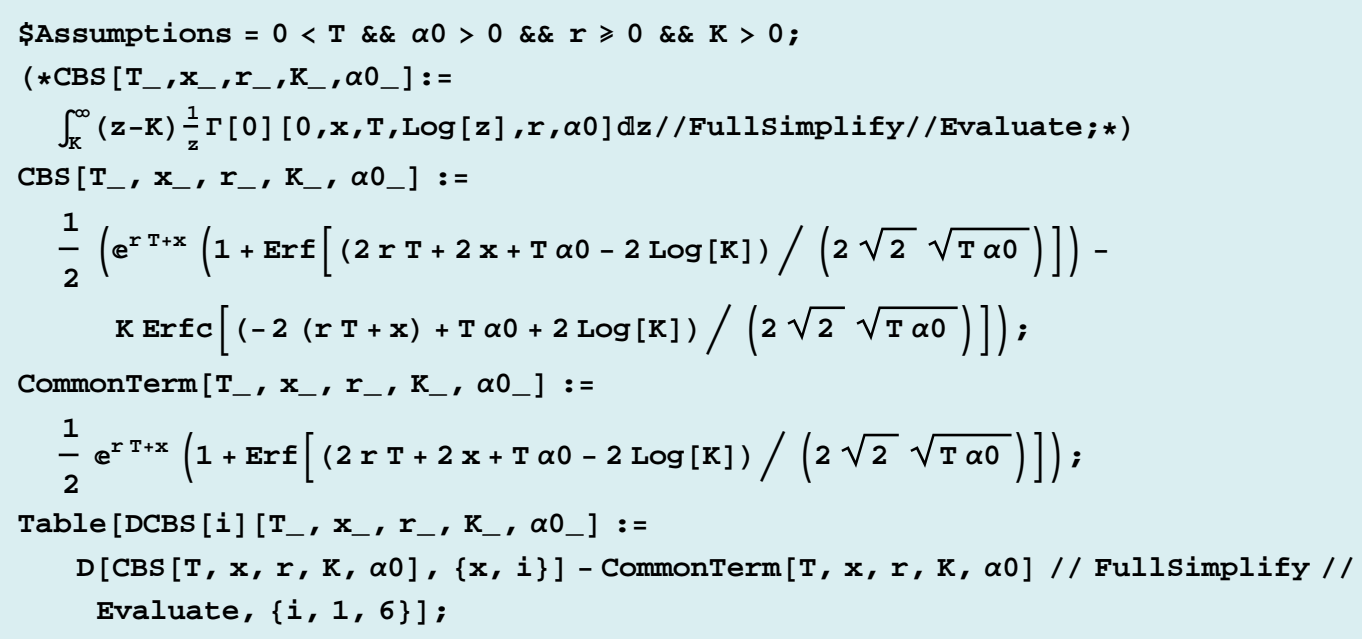

Next we recall that $\bar{x}=x$ and we compute

$$
\begin{gathered}
u^{1}(0, x)=C_{\mathrm{BS}}(0, x)+Q^{1}(x) \\
u^{2}(0, x)=C_{\mathrm{BS}}(0, x)+Q^{1}(x)+Q^{2}(x)
\end{gathered}
$$

where

$$
\begin{aligned}
& Q^{1}(x)=J^{1}(0, T, x) C_{\mathrm{BS}}(0, x) \\
& Q^{2}(x)=J^{2}(0, T, x) C_{\mathrm{BS}}(0, x)
\end{aligned}
$$

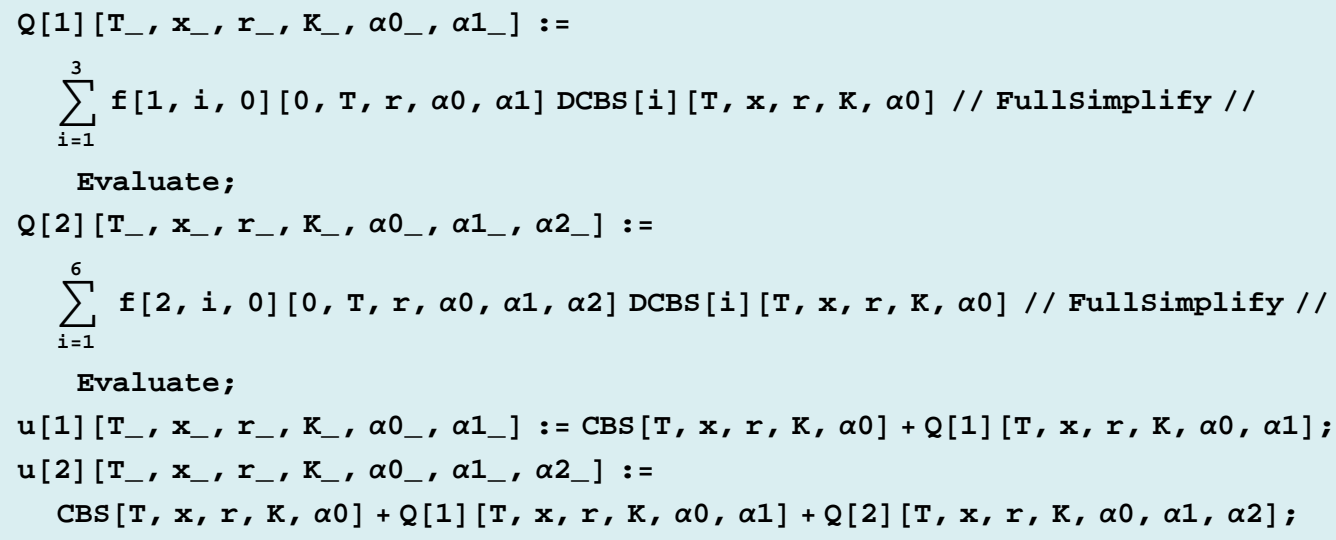

Eventually, the zeroth, first and second order approximation of the Call price are given by

$$
\begin{gathered}
C^{0}\left(0, S_{t}\right)=e^{-r T} C_{\mathrm{BS}}(0, x) \\
C^{1}\left(0, S_{t}\right)=e^{-r T} u^{1}\left(0, \log S_{t}\right) \\
C^{2}\left(0, S_{t}\right)=e^{-r T} u^{2}\left(0, \log S_{t}\right)
\end{gathered}
$$

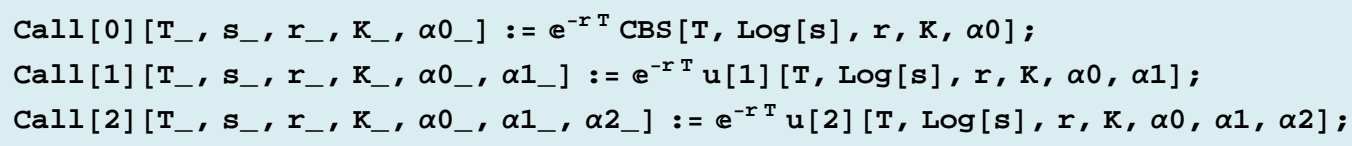

\section{Explicit formulae for the 4th order approximations}

Now we give the formulas of the approximations, up to the 4th order, of the density and the price of a Call option in a generic LV model.

\section{Density Approximations}

$$
\Gamma[0]\left[t_{-}, x_{-}, T_{-}, z_{-}, r_{-}, \alpha 0_{-}\right]:=\frac{e^{-\frac{\left(x-z+(-t+T)\left(x-\frac{\alpha}{2}\right)\right)^{2}}{2(-t+T) \alpha 0}}}{\sqrt{2 \pi} \sqrt{(-t+T) \alpha 0}} ;
$$




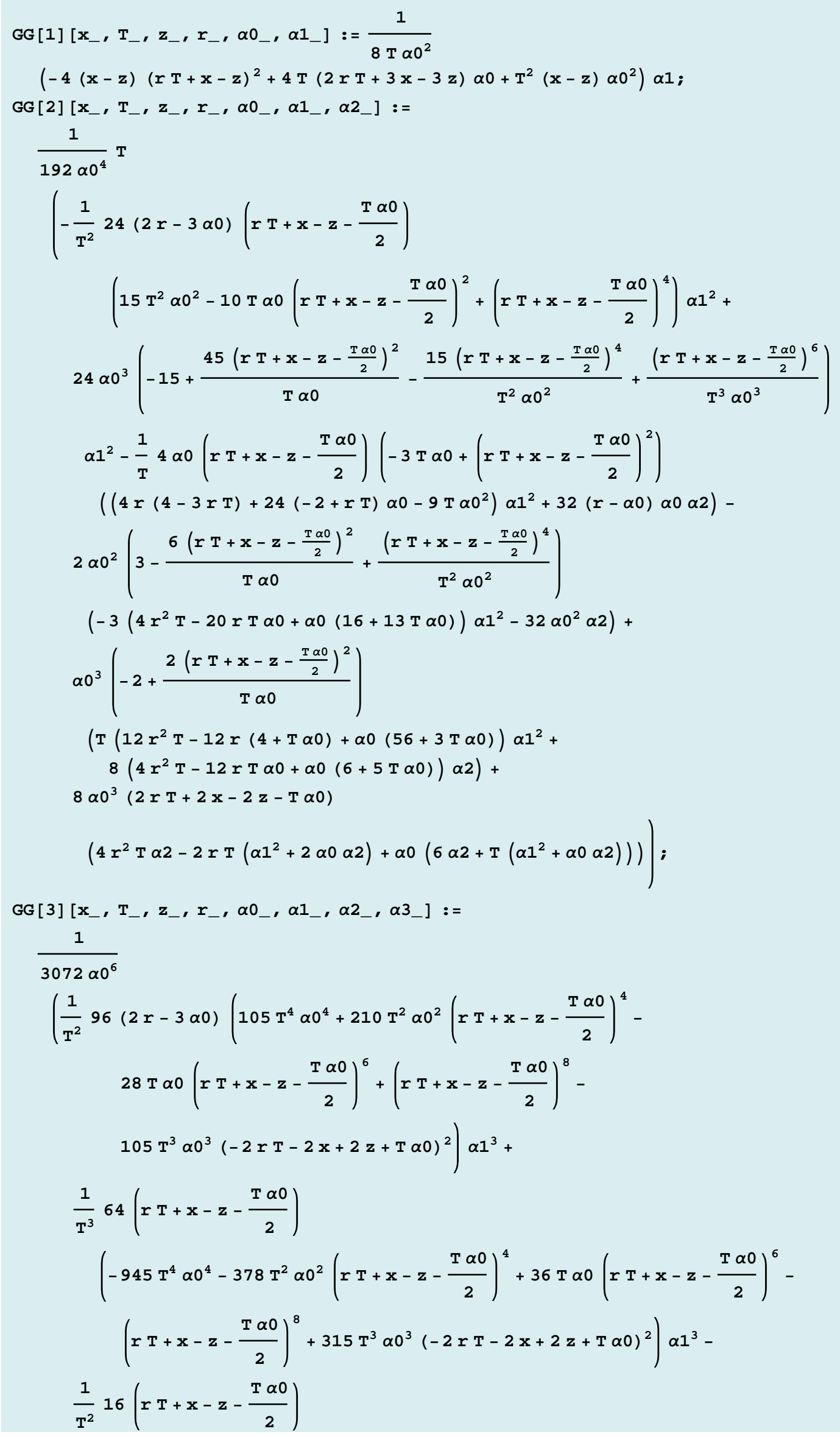




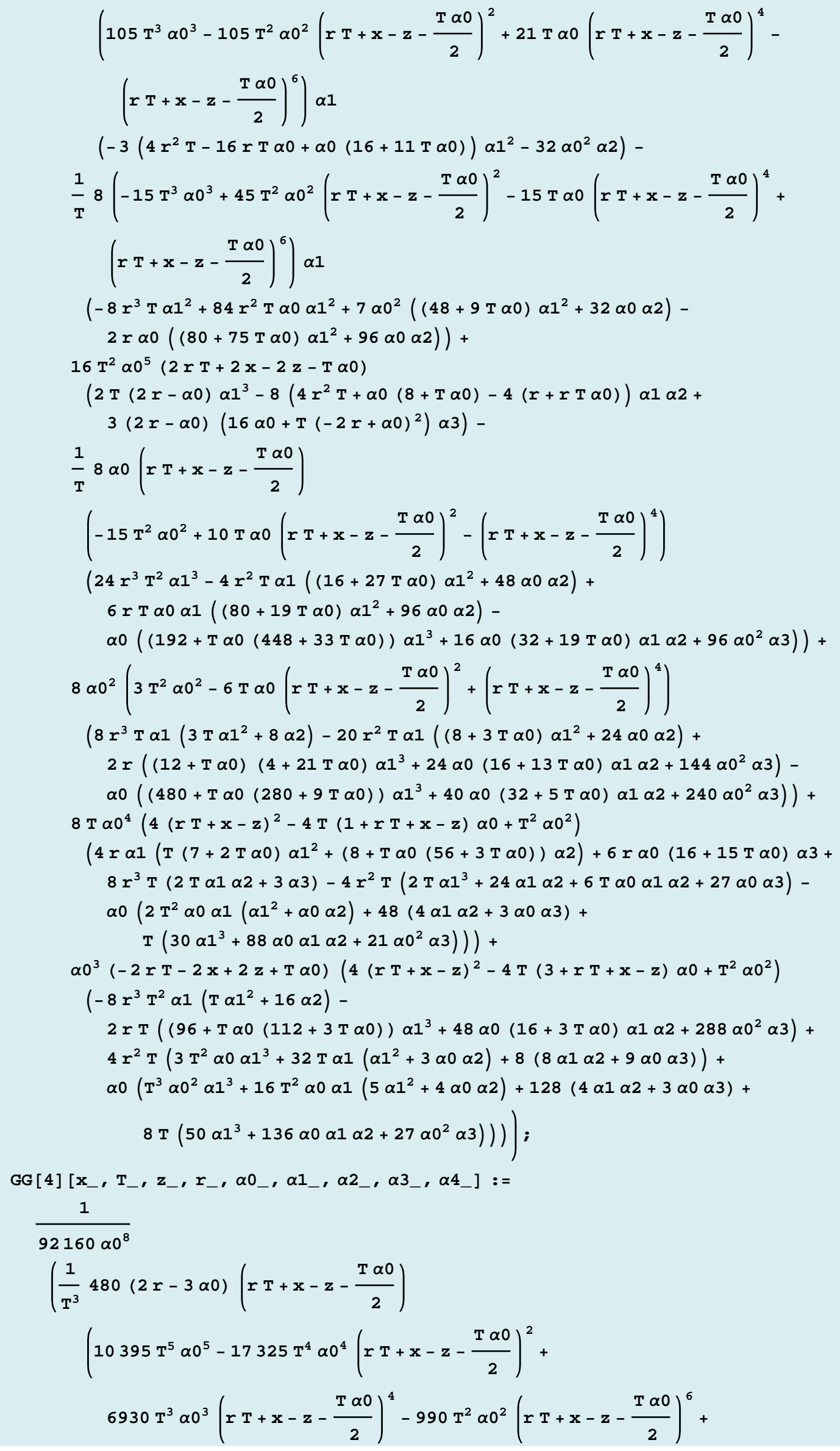


$\left.55 T \alpha 0\left(r T+x-z-\frac{T \alpha 0}{2}\right)^{8}-\left(r T+x-z-\frac{T \alpha 0}{2}\right)^{10}\right) \alpha 1^{4}+$

$\frac{1}{T^{4}} 240\left(10395 T^{6} \alpha 0^{6}-62370 T^{5} \alpha 0^{5}\left(r T+x-z-\frac{T \alpha 0}{2}\right)^{2}+\right.$

$51975 \mathrm{~T}^{4} \alpha 0^{4}\left(\mathrm{r} \mathrm{T}+\mathrm{x}-\mathrm{z}-\frac{\mathrm{T} \alpha 0}{2}\right)^{4}-13860 \mathrm{~T}^{3} \alpha 0^{3}\left(\mathrm{r} \mathrm{T}+\mathrm{x}-\mathrm{z}-\frac{\mathrm{T} \alpha 0}{2}\right)^{6}+$

$1485 T^{2} \alpha 0^{2}\left(r T+x-z-\frac{T \alpha 0}{2}\right)^{8}-66 T \alpha 0\left(r T+x-z-\frac{T \alpha 0}{2}\right)^{10}+$

$\left.\left(r T+x-z-\frac{T \alpha 0}{2}\right)^{12}\right) \alpha 1^{4}-$

$\frac{1}{T^{3}} 120\left(-945 T^{5} \alpha 0^{5}+4725 T^{4} \alpha 0^{4}\left(r T+x-z-\frac{T \alpha 0}{2}\right)^{2}-\right.$

$3150 \mathrm{~T}^{3} \alpha 0^{3}\left(\mathrm{r} T+\mathrm{x}-\mathrm{z}-\frac{\mathrm{T} \alpha 0}{2}\right)^{4}+630 \mathrm{~T}^{2} \alpha 0^{2}\left(\mathrm{r} \mathrm{T}+\mathrm{x}-\mathrm{z}-\frac{\mathrm{T} \alpha 0}{2}\right)^{6}-$

$\left.45 \mathrm{~T} \alpha 0\left(\mathrm{r} T+\mathbf{x}-\mathrm{z}-\frac{\mathrm{T} \alpha 0}{2}\right)^{8}+\left(\mathrm{r} \mathrm{T}+\mathrm{x}-\mathrm{z}-\frac{\mathrm{T} \alpha 0}{2}\right)^{10}\right) \alpha 1^{2}$

$\left(\left(-12 r^{2} T+44 r T \alpha 0-\alpha 0(48+31 T \alpha 0)\right) \alpha 1^{2}-32 \alpha 0^{2} \alpha 2\right)-$

$\frac{1}{T^{2}} 120\left(r T+x-z-\frac{T \alpha 0}{2}\right)$

$\left(-945 \mathrm{~T}^{4} \alpha 0^{4}-378 \mathrm{~T}^{2} \alpha 0^{2}\left(\mathrm{r} T+\mathrm{x}-\mathrm{z}-\frac{\mathrm{T} \alpha 0}{2}\right)^{4}+36 \mathrm{~T} \alpha 0\left(\mathrm{r} \mathrm{T}+\mathrm{x}-\mathrm{z}-\frac{\mathrm{T} \alpha 0}{2}\right)^{6}-\right.$

$\left.\left(\mathrm{r} T+\mathrm{x}-\mathrm{z}-\frac{\mathrm{T} \alpha 0}{2}\right)^{8}+315 \mathrm{~T}^{3} \alpha 0^{3}(-2 \mathrm{r}-2 \mathrm{x}+2 \mathrm{z}+\mathrm{T} \alpha 0)^{2}\right) \alpha 1^{2}$

$\left(-8 r^{3} T \alpha 1^{2}+60 r^{2} T \alpha 0 \alpha 1^{2}+5 \alpha 0^{2}\left((48+9 T \alpha 0) \alpha 1^{2}+32 \alpha 0 \alpha 2\right)-\right.$

$\left.2 r \alpha 0\left((64+51 \mathrm{~T} \alpha 0) \alpha 1^{2}+64 \alpha 0 \alpha 2\right)\right)-$

$\frac{1}{T} 20 \alpha 0\left(r T+x-z-\frac{T \alpha 0}{2}\right)$

$\left(105 T^{3} \alpha 0^{3}-105 T^{2} \alpha 0^{2}\left(r T+x-z-\frac{T \alpha 0}{2}\right)^{2}+21 T \alpha 0\left(r T+x-z-\frac{T \alpha 0}{2}\right)^{4}-\right.$

$\left.\left(r T+x-z-\frac{T \alpha 0}{2}\right)^{6}\right)$

$\left(48 \mathrm{r}^{4} \mathrm{~T}^{2} \alpha 1^{4}-192 \mathrm{r}^{3} \mathrm{~T} \alpha 1^{2}\left((1+2 \mathrm{~T} \alpha 0) \alpha 1^{2}+4 \alpha 0 \alpha 2\right)+\right.$

$24 \mathrm{r}^{2} \mathrm{~T} \alpha 0 \alpha 1^{2}\left((112+33 \mathrm{~T} \alpha 0) \alpha 1^{2}+192 \alpha 0 \alpha 2\right)-$

$16 \mathrm{r} \alpha 0\left(3(52+\mathrm{T} \alpha 0(131+12 \mathrm{~T} \alpha 0)) \alpha 1^{4}+44 \alpha 0(13+9 \mathrm{~T} \alpha 0) \alpha 1^{2} \alpha 2+\right.$

$\left.64 \alpha 0^{2} \alpha 2^{2}+144 \alpha 0^{2} \alpha 1 \alpha 3\right)+$

$\alpha 0^{2}\left(3(2304+5 \mathrm{~T} \alpha 0(224+9 \mathrm{~T} \alpha 0)) \alpha 1^{4}+384 \alpha 0(37+6 \mathrm{~T} \alpha 0) \alpha 1^{2} \alpha 2+\right.$ $\left.\left.1024 \alpha 0^{2} \alpha 2^{2}+2304 \alpha 0^{2} \alpha 1 \alpha 3\right)\right)+$

$\frac{1}{T^{2}} 5\left(105 T^{4} \alpha 0^{4}+210 T^{2} \alpha 0^{2}\left(r T+x-z-\frac{T \alpha 0}{2}\right)^{4}-28 T \alpha 0\left(r T+x-z-\frac{T \alpha 0}{2}\right)^{6}+\right.$

$\left.\left(r T+x-z-\frac{T \alpha 0}{2}\right)^{8}-105 T^{3} \alpha 0^{3}(-2 r T-2 x+2 z+T \alpha 0)^{2}\right)$

$\left(48 r^{4} T^{2} \alpha 1^{4}-864 r^{3} T^{2} \alpha 0 \alpha 1^{4}-72 r T \alpha 0^{2} \alpha 1^{2}\left((192+43 T \alpha 0) \alpha 1^{2}+192 \alpha 0 \alpha 2\right)+\right.$

$24 \mathrm{r}^{2} \mathrm{~T} \alpha 0 \alpha 1^{2}\left((112+123 \mathrm{~T} \alpha 0) \alpha 1^{2}+192 \alpha 0 \alpha 2\right)+$

$\alpha 0^{2}\left(3(2304+\mathrm{T} \alpha 0(4000+321 \mathrm{~T} \alpha 0)) \alpha 1^{4}+384 \alpha 0(37+21 \mathrm{~T} \alpha 0) \alpha 1^{2} \alpha 2+\right.$ $\left.\left.1024 \alpha 0^{2} \alpha 2^{2}+2304 \alpha 0^{2} \alpha 1 \alpha 3\right)\right)-$ 


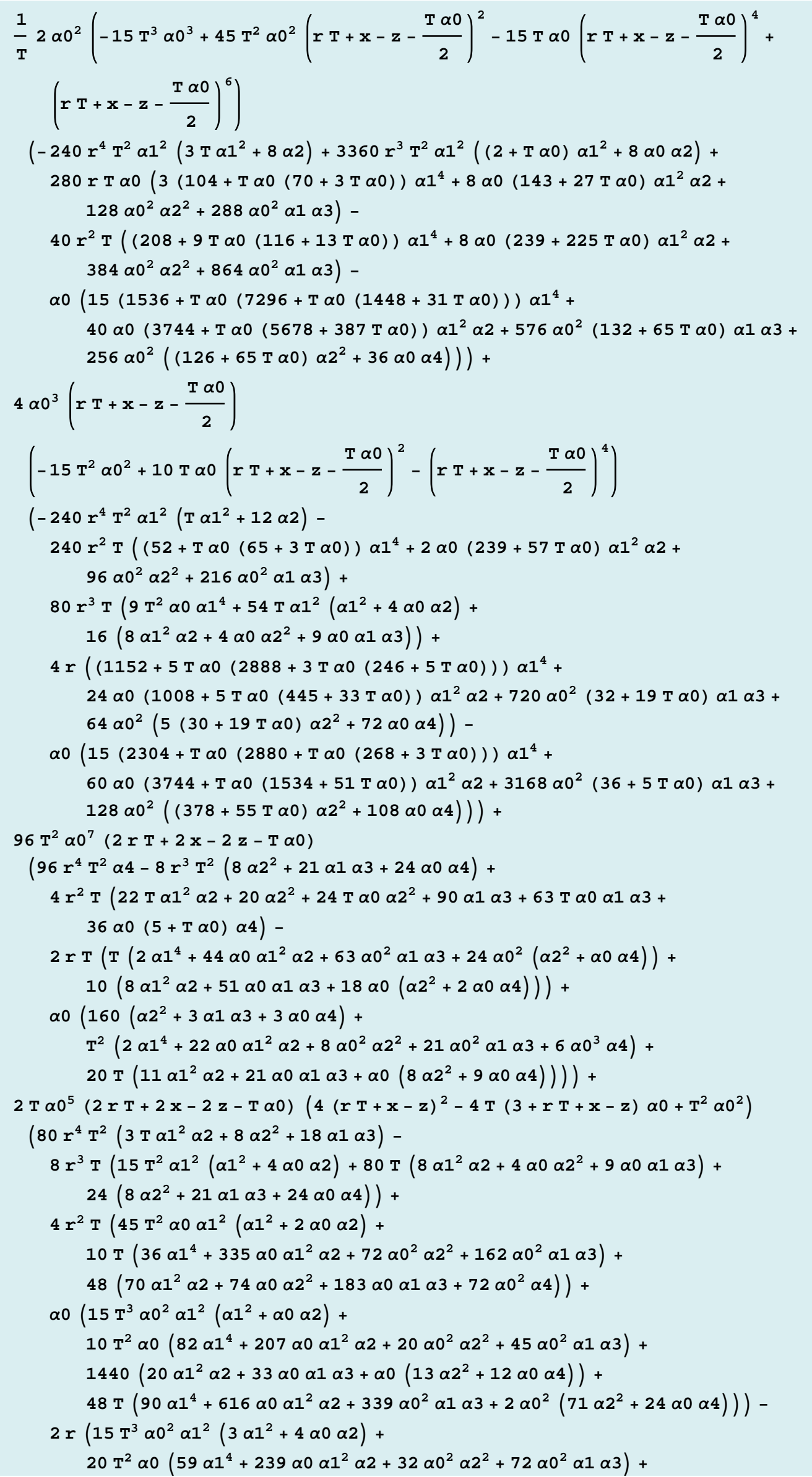




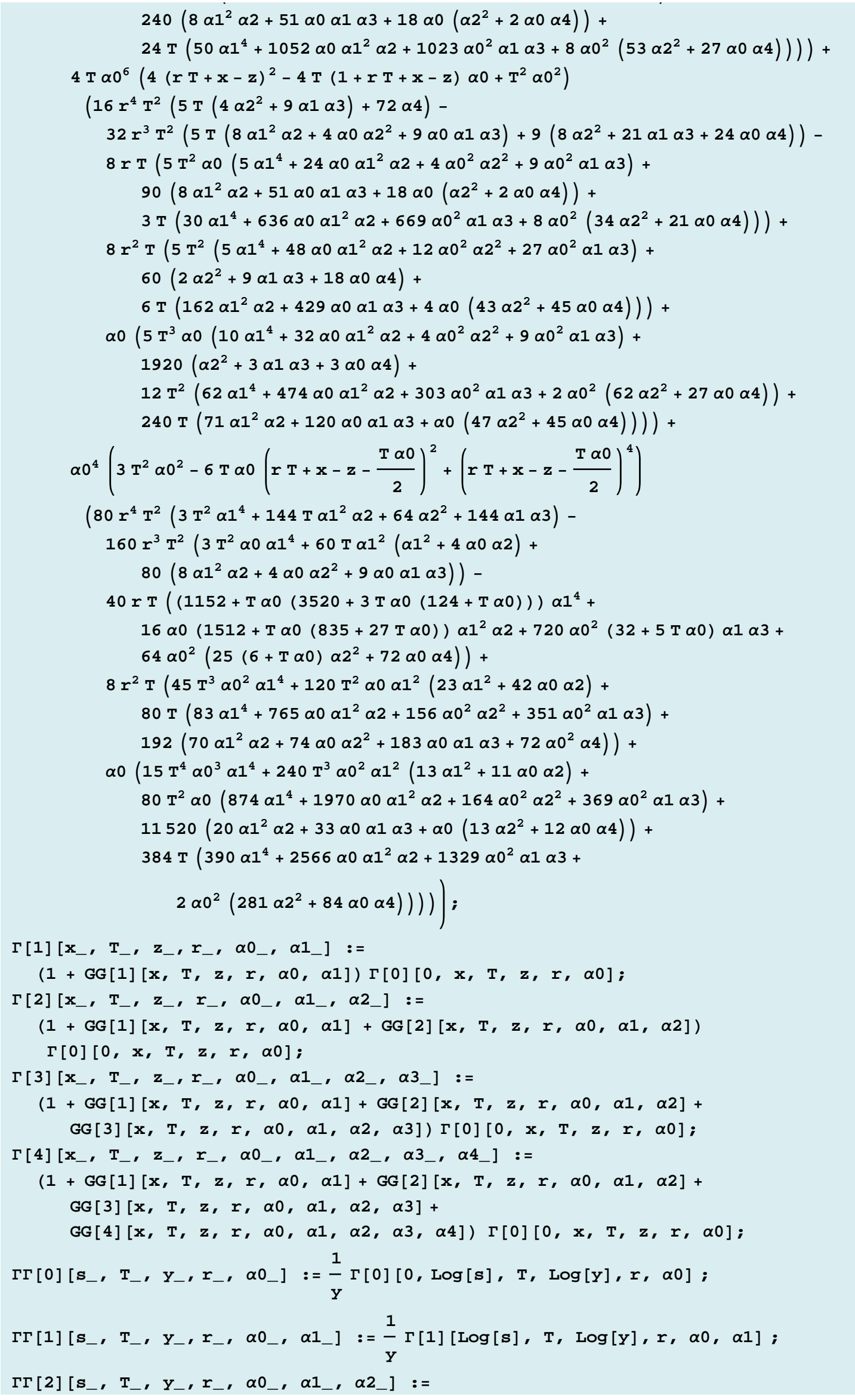




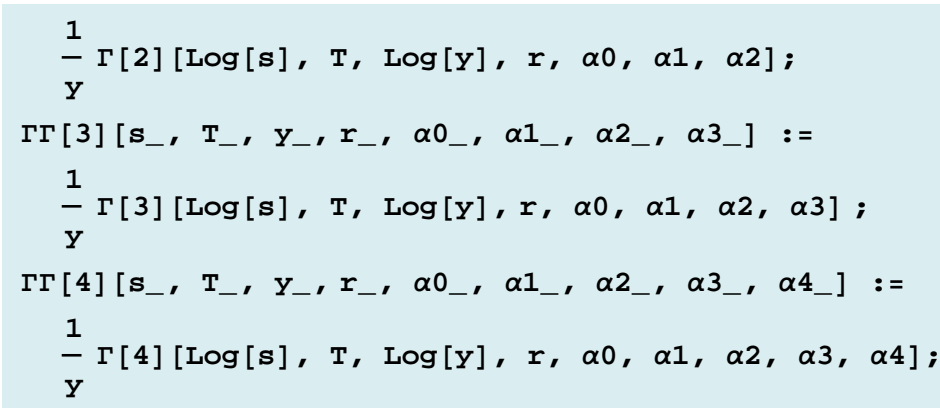

\section{Call price Approximations}

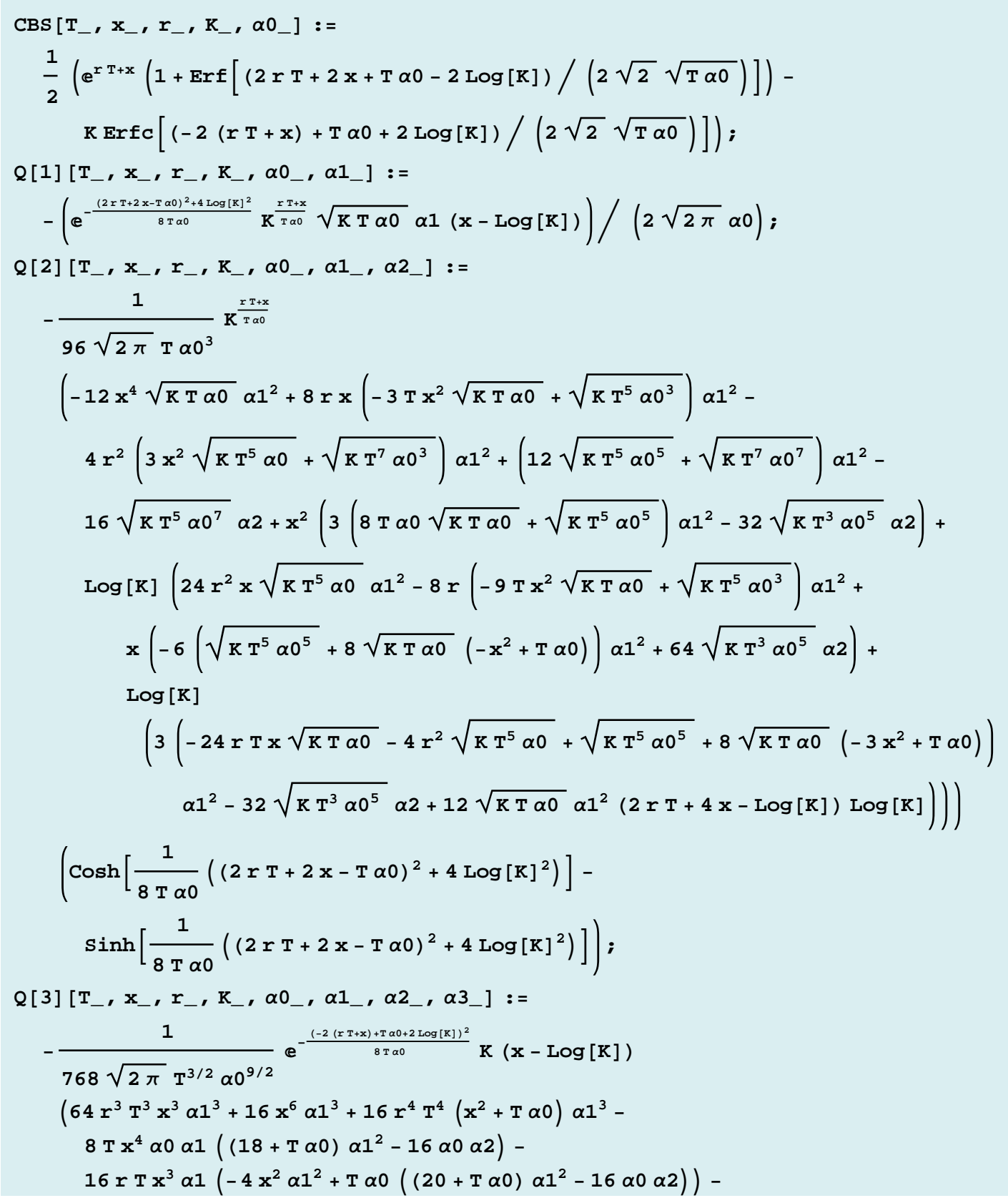




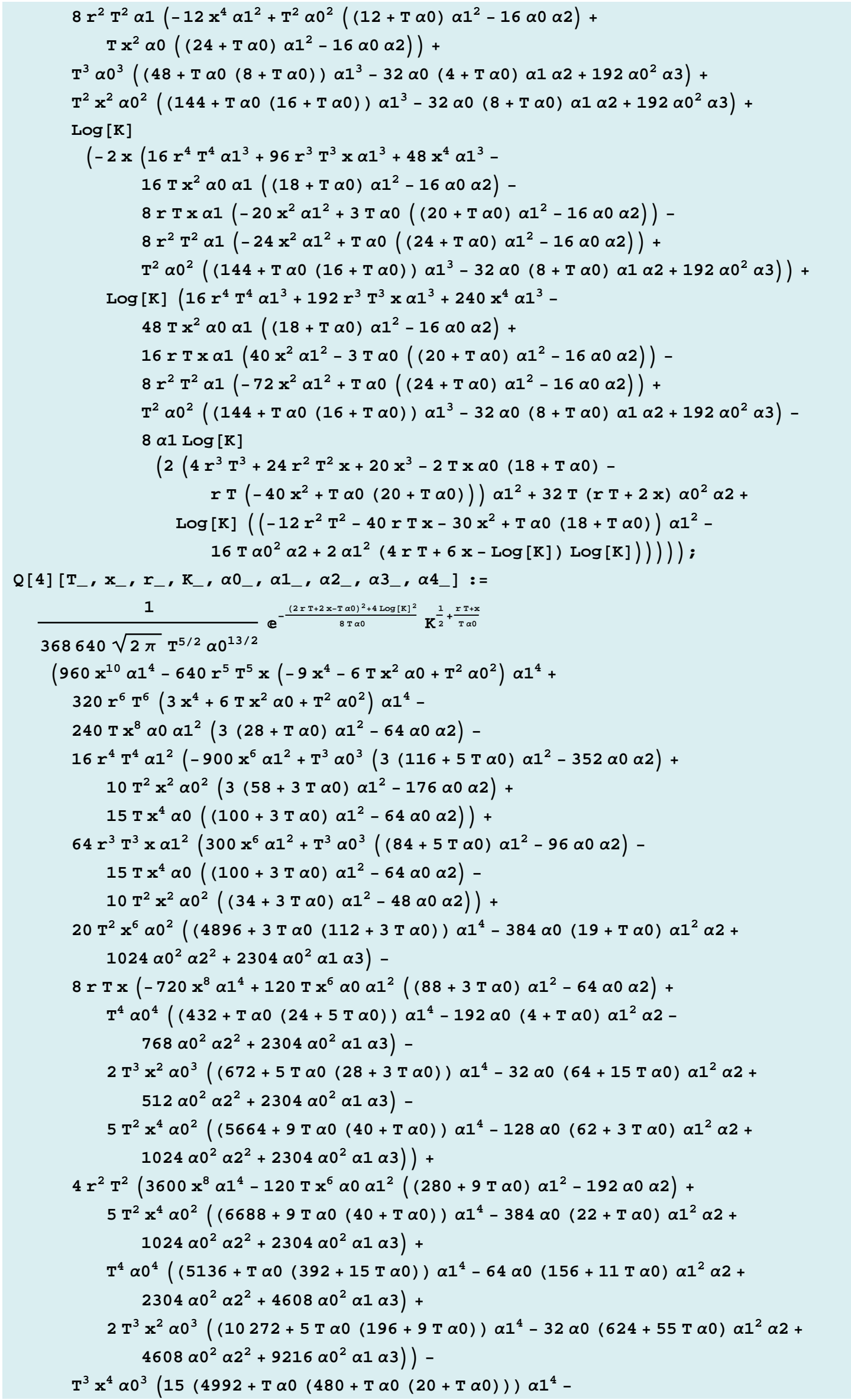


$320 \alpha 0(504+\mathrm{T} \alpha 0(44+3 \mathrm{~T} \alpha 0)) \alpha 1^{2} \alpha 2+2304 \alpha 0^{2}(36+5 \mathrm{~T} \alpha 0) \alpha 1 \alpha 3+$ $\left.1024 \alpha 0^{2}\left((48+5 \mathrm{~T} \alpha 0) \alpha 2^{2}-72 \alpha 0 \alpha 4\right)\right)-$

$T^{5} \alpha 0^{5}\left((8640+T \alpha 0(720+T \alpha 0(44+5 T \alpha 0))) \alpha 1^{4}-\right.$

$32 \alpha 0(720+T \alpha 0(72+11 T \alpha 0)) \alpha 1^{2} \alpha 2+4608 \alpha 0^{2}(4+T \alpha 0) \alpha 1 \alpha 3+$

$\left.2304 \alpha 0^{2}\left((4+\mathrm{T} \alpha 0) \alpha 2^{2}-16 \alpha 0 \alpha 4\right)\right)-$

$2 \mathrm{~T}^{4} \mathrm{x}^{2} \alpha 0^{4}\left(5(2592+\mathrm{T} \alpha 0(288+\mathrm{T} \alpha 0(22+3 \mathrm{~T} \alpha 0))) \alpha 1^{4}-\right.$

$16 \alpha 0(2160+\mathrm{T} \alpha 0(288+55 \mathrm{~T} \alpha 0)) \alpha 1^{2} \alpha 2+9216 \alpha 0^{2}(3+\mathrm{T} \alpha 0) \alpha 1 \alpha 3+$

$\left.4608 \alpha 0^{2}\left((3+T \alpha 0) \alpha 2^{2}-12 \alpha 0 \alpha 4\right)\right)+$

Log $[\mathrm{K}]$

$\left(-4\left(960 r^{6} T^{6} x\left(x^{2}+T \alpha 0\right) \alpha 1^{4}-160 r^{5} T^{5}\left(-45 x^{4}-18 T x^{2} \alpha 0+T^{2} \alpha 0^{2}\right) \alpha 1^{4}-\right.\right.$

$80 \mathrm{r}^{4} \mathrm{~T}^{4} \times \alpha 1^{2}\left(-270 \mathrm{x}^{4} \alpha 1^{2}+\mathrm{T}^{2} \alpha 0^{2}\left(3(58+3 \mathrm{~T} \alpha 0) \alpha 1^{2}-176 \alpha 0 \alpha 2\right)+\right.$

$\left.3 T \mathbf{x}^{2} \alpha 0\left((100+3 \mathrm{~T} \alpha 0) \alpha 1^{2}-64 \alpha 0 \alpha 2\right)\right)+$

$16 \mathrm{r}^{3} \mathrm{~T}^{3} \alpha 1^{2}\left(2100 \mathrm{x}^{6} \alpha 1^{2}+\mathrm{T}^{3} \alpha 0^{3}\left((84+5 \mathrm{~T} \alpha 0) \alpha 1^{2}-96 \alpha 0 \alpha 2\right)-\right.$

$75 \mathrm{~T} \mathrm{x}^{4} \alpha 0\left((100+3 \mathrm{~T} \alpha 0) \alpha 1^{2}-64 \alpha 0 \alpha 2\right)-$

$\left.30 \mathrm{~T}^{2} \mathrm{x}^{2} \alpha 0^{2}\left((34+3 \mathrm{~T} \alpha 0) \alpha 1^{2}-48 \alpha 0 \alpha 2\right)\right)-$

$2 \mathrm{rT}\left(-6480 \mathrm{x}^{8} \alpha 1^{4}+840 \mathrm{~T} \mathrm{x^{6 }} \alpha 0 \alpha 1^{2}\left((88+3 \mathrm{~T} \alpha 0) \alpha 1^{2}-64 \alpha 0 \alpha 2\right)+\right.$

$\mathrm{T}^{4} \alpha 0^{4}\left((432+\mathrm{T} \alpha 0(24+5 \mathrm{~T} \alpha 0)) \alpha 1^{4}-192 \alpha 0(4+\mathrm{T} \alpha 0) \alpha 1^{2} \alpha 2-\right.$

$\left.768 \alpha 0^{2} \alpha 2^{2}+2304 \alpha 0^{2} \alpha 1 \alpha 3\right)-$

$6 \mathrm{~T}^{3} \mathrm{x}^{2} \alpha 0^{3}\left((672+5 \mathrm{~T} \alpha 0(28+3 \mathrm{~T} \alpha 0)) \alpha 1^{4}-32 \alpha 0(64+15 \mathrm{~T} \alpha 0) \alpha 1^{2} \alpha 2+\right.$ $\left.512 \alpha 0^{2} \alpha 2^{2}+2304 \alpha 0^{2} \alpha 1 \alpha 3\right)-$

$25 \mathrm{~T}^{2} \mathrm{x}^{4} \alpha 0^{2}\left((5664+9 \mathrm{~T} \alpha 0(40+\mathrm{T} \alpha 0)) \alpha 1^{4}-128 \alpha 0(62+3 \mathrm{~T} \alpha 0) \alpha 1^{2} \alpha 2+\right.$ $\left.\left.1024 \alpha 0^{2} \alpha 2^{2}+2304 \alpha 0^{2} \alpha 1 \alpha 3\right)\right)+$

$4 \mathrm{r}^{2} \mathrm{~T}^{2} \mathrm{x}\left(7200 \mathrm{x}^{6} \alpha 1^{4}-180 \mathrm{~T} \mathrm{x^{4 }} \alpha 0 \alpha 1^{2}\left((280+9 \mathrm{~T} \alpha 0) \alpha 1^{2}-192 \alpha 0 \alpha 2\right)+\right.$

$5 \mathrm{~T}^{2} \mathrm{x}^{2} \alpha 0^{2}\left((6688+9 \mathrm{~T} \alpha 0(40+\mathrm{T} \alpha 0)) \alpha 1^{4}-384 \alpha 0(22+\mathrm{T} \alpha 0) \alpha 1^{2} \alpha 2+\right.$ $\left.1024 \alpha 0^{2} \alpha 2^{2}+2304 \alpha 0^{2} \alpha 1 \alpha 3\right)+$

$\mathrm{T}^{3} \alpha 0^{3}\left((10272+5 \mathrm{~T} \alpha 0(196+9 \mathrm{~T} \alpha 0)) \alpha 1^{4}-32 \alpha 0(624+55 \mathrm{~T} \alpha 0) \alpha 1^{2} \alpha 2+\right.$ $\left.\left.4608 \alpha 0^{2} \alpha 2^{2}+9216 \alpha 0^{2} \alpha 1 \alpha 3\right)\right)+$

$x\left(2400 x^{8} \alpha 1^{4}-480 T x^{6} \alpha 0 \alpha 1^{2}\left(3(28+T \alpha 0) \alpha 1^{2}-64 \alpha 0 \alpha 2\right)+\right.$

$30 \mathrm{~T}^{2} \mathrm{x}^{4} \alpha 0^{2}\left((4896+3 \mathrm{~T} \alpha 0(112+3 \mathrm{~T} \alpha 0)) \alpha 1^{4}-\right.$

$\left.384 \alpha 0(19+\mathrm{T} \alpha 0) \alpha 1^{2} \alpha 2+1024 \alpha 0^{2} \alpha 2^{2}+2304 \alpha 0^{2} \alpha 1 \alpha 3\right)-$

$T^{3} x^{2} \alpha 0^{3}\left(15(4992+T \alpha 0(480+T \alpha 0(20+T \alpha 0))) \alpha 1^{4}-\right.$

$320 \alpha 0(504+T \alpha 0(44+3 T \alpha 0)) \alpha 1^{2} \alpha 2+$

$2304 \alpha 0^{2}(36+5 \mathrm{~T} \alpha 0) \alpha 1 \alpha 3+1024 \alpha 0^{2}$

$\left.\left((48+5 \mathrm{~T} \alpha 0) \alpha 2^{2}-72 \alpha 0 \alpha 4\right)\right)-$

$T^{4} \alpha 0^{4}\left(5(2592+T \alpha 0(288+T \alpha 0(22+3 T \alpha 0))) \alpha 1^{4}-\right.$

$16 \alpha 0(2160+\mathrm{T} \alpha 0(288+55 \mathrm{~T} \alpha 0)) \alpha 1^{2} \alpha 2+$

$\left.\left.\left.9216 \alpha 0^{2}(3+T \alpha 0) \alpha 1 \alpha 3+4608 \alpha 0^{2}\left((3+T \alpha 0) \alpha 2^{2}-12 \alpha 0 \alpha 4\right)\right)\right)\right)+$

$\log [\mathrm{K}]$

(2 $\left(21600 x^{8} \alpha 1^{4}+960 r^{6} T^{6}\left(3 x^{2}+T \alpha 0\right) \alpha 1^{4}+5760 r^{5} T^{5} x\left(5 x^{2}+T \alpha 0\right) \alpha 1^{4}-\right.$

$3360 \mathrm{~T} \mathrm{x}^{6} \alpha 0 \alpha 1^{2}\left(3(28+\mathrm{T} \alpha 0) \alpha 1^{2}-64 \alpha 0 \alpha 2\right)-$

$80 r^{4} T^{4} \alpha 1^{2}\left(-1350 x^{4} \alpha 1^{2}+T^{2} \alpha 0^{2}\left(3(58+3 T \alpha 0) \alpha 1^{2}-176 \alpha 0 \alpha 2\right)+\right.$

$\left.9 \mathrm{~T} \mathrm{x}^{2} \alpha 0\left((100+3 \mathrm{~T} \alpha 0) \alpha 1^{2}-64 \alpha 0 \alpha 2\right)\right)-$

$960 \mathrm{r}^{3} \mathrm{~T}^{3} \mathrm{x} \alpha 1^{2}\left(-210 \mathrm{x}^{4} \alpha 1^{2}+5 \mathrm{~T} \mathrm{x}^{2} \alpha 0\left((100+3 \mathrm{~T} \alpha 0) \alpha 1^{2}-64 \alpha 0 \alpha 2\right)+\right.$

$\left.\mathrm{T}^{2} \alpha 0^{2}\left((34+3 \mathrm{~T} \alpha 0) \alpha 1^{2}-48 \alpha 0 \alpha 2\right)\right)+$

$150 \mathrm{~T}^{2} \mathrm{x}^{4} \alpha 0^{2}\left((4896+3 \mathrm{~T} \alpha 0(112+3 \mathrm{~T} \alpha 0)) \alpha 1^{4}-\right.$

$\left.384 \alpha 0(19+\mathrm{T} \alpha 0) \alpha 1^{2} \alpha 2+1024 \alpha 0^{2} \alpha 2^{2}+2304 \alpha 0^{2} \alpha 1 \alpha 3\right)+$

$8 \mathrm{r} \mathrm{T}\left(12960 \mathrm{x}^{6} \alpha 1^{4}-1260 \mathrm{~T} \mathrm{x}^{4} \alpha 0 \alpha 1^{2}\left((88+3 \mathrm{~T} \alpha 0) \alpha 1^{2}-64 \alpha 0 \alpha 2\right)+\right.$ $3 \mathrm{~T}^{3} \alpha 0^{3}\left((672+5 \mathrm{~T} \alpha 0(28+3 \mathrm{~T} \alpha 0)) \alpha 1^{4}-\right.$

$\left.32 \alpha 0(64+15 \mathrm{~T} \alpha 0) \alpha 1^{2} \alpha 2+512 \alpha 0^{2} \alpha 2^{2}+2304 \alpha 0^{2} \alpha 1 \alpha 3\right)+$ $25 \mathrm{~T}^{2} \mathrm{x}^{2} \alpha 0^{2}\left((5664+9 \mathrm{~T} \alpha 0(40+\mathrm{T} \alpha 0)) \alpha 1^{4}-\right.$ 
$\left.\left.128 \alpha 0(62+3 \mathrm{~T} \alpha 0) \alpha 1^{2} \alpha 2+1024 \alpha 0^{2} \alpha 2^{2}+2304 \alpha 0^{2} \alpha 1 \alpha 3\right)\right)+$ $4 \mathrm{r}^{2} \mathrm{~T}^{2}\left(50400 \mathrm{x}^{6} \alpha 1^{4}-900 \mathrm{~T} \mathrm{x}^{4} \alpha 0 \alpha 1^{2}\left((280+9 \mathrm{~T} \alpha 0) \alpha 1^{2}-192 \alpha 0 \alpha 2\right)+\right.$ $15 \mathrm{~T}^{2} \mathbf{x}^{2} \alpha 0^{2}\left((6688+9 \mathrm{~T} \alpha 0(40+\mathrm{T} \alpha 0)) \alpha 1^{4}-\right.$

$\left.384 \alpha 0(22+\mathrm{T} \alpha 0) \alpha 1^{2} \alpha 2+1024 \alpha 0^{2} \alpha 2^{2}+2304 \alpha 0^{2} \alpha 1 \alpha 3\right)+$ $T^{3} \alpha 0^{3}\left((10272+5 T \alpha 0(196+9 T \alpha 0)) \alpha 1^{4}-\right.$

$\left.\left.32 \alpha 0(624+55 \mathrm{~T} \alpha 0) \alpha 1^{2} \alpha 2+4608 \alpha 0^{2} \alpha 2^{2}+9216 \alpha 0^{2} \alpha 1 \alpha 3\right)\right)-$ $3 \mathbf{T}^{3} \mathbf{x}^{2} \alpha 0^{3}\left(15(4992+T \alpha 0(480+T \alpha 0(20+T \alpha 0))) \alpha 1^{4}-\right.$

$320 \alpha 0(504+\mathrm{T} \alpha 0(44+3 \mathrm{~T} \alpha 0)) \alpha 1^{2} \alpha 2+$

$2304 \alpha 0^{2}(36+5 \mathrm{~T} \alpha 0) \alpha 1 \alpha 3+1024 \alpha 0^{2}$

$\left.\left((48+5 \mathrm{~T} \alpha 0) \alpha 2^{2}-72 \alpha 0 \alpha 4\right)\right)-$

$\mathrm{T}^{4} \alpha 0^{4}\left(5(2592+\mathrm{T} \alpha 0(288+\mathrm{T} \alpha 0(22+3 \mathrm{~T} \alpha 0))) \alpha 1^{4}-\right.$

$16 \alpha 0(2160+\mathrm{T} \alpha 0(288+55 \mathrm{~T} \alpha 0)) \alpha 1^{2} \alpha 2+$

$\left.\left.9216 \alpha 0^{2}(3+T \alpha 0) \alpha 1 \alpha 3+4608 \alpha 0^{2}\left((3+T \alpha 0) \alpha 2^{2}-12 \alpha 0 \alpha 4\right)\right)\right)+$

$\log [K]\left(-4\left(960 r^{6} T^{6} \times \alpha 1^{4}+960 r^{5} T^{5}\left(15 x^{2}+T \alpha 0\right) \alpha 1^{4}-\right.\right.$

$240 \mathrm{r}^{4} \mathrm{~T}^{4} \times \alpha 1^{2}\left(-300 \mathrm{x}^{2} \alpha 1^{2}+\mathrm{T} \alpha 0\left((100+3 \mathrm{~T} \alpha 0) \alpha 1^{2}-64 \alpha 0 \alpha 2\right)\right)-$ $160 \mathrm{r}^{3} \mathrm{~T}^{3} \alpha 1^{2}\left(-1050 \mathrm{x}^{4} \alpha 1^{2}+15 \mathrm{~T} \mathrm{x^{2 }} \alpha 0\right.$

$\left((100+3 T \alpha 0) \alpha 1^{2}-64 \alpha 0 \alpha 2\right)+$

$\left.T^{2} \alpha 0^{2}\left((34+3 T \alpha 0) \alpha 1^{2}-48 \alpha 0 \alpha 2\right)\right)+$

$20 \mathrm{r}^{2} \mathrm{~T}^{2} \mathrm{x}\left(10080 \mathrm{x}^{4} \alpha 1^{4}-120 \mathrm{~T} \mathrm{x}^{2} \alpha 0 \alpha 1^{2}\right.$

$\left((280+9 \mathrm{~T} \alpha 0) \alpha 1^{2}-192 \alpha 0 \alpha 2\right)+$

$\mathrm{T}^{2} \alpha 0^{2}\left((6688+9 \mathrm{~T} \alpha 0(40+\mathrm{T} \alpha 0)) \alpha 1^{4}-\right.$

$\left.\left.384 \alpha 0(22+\mathrm{T} \alpha 0) \alpha 1^{2} \alpha 2+1024 \alpha 0^{2} \alpha 2^{2}+2304 \alpha 0^{2} \alpha 1 \alpha 3\right)\right)+$ $4 \mathrm{r} T\left(30240 \mathrm{x}^{6} \alpha 1^{4}-2100 \mathrm{~T} \mathrm{x}^{4} \alpha 0 \alpha 1^{2}\left((88+3 \mathrm{~T} \alpha 0) \alpha 1^{2}-64 \alpha 0 \alpha 2\right)+\right.$

$\mathrm{T}^{3} \alpha 0^{3}\left((672+5 \mathrm{~T} \alpha 0(28+3 \mathrm{~T} \alpha 0)) \alpha 1^{4}-\right.$

$\left.32 \alpha 0(64+15 \mathrm{~T} \alpha 0) \alpha 1^{2} \alpha 2+512 \alpha 0^{2} \alpha 2^{2}+2304 \alpha 0^{2} \alpha 1 \alpha 3\right)+$ $25 \mathrm{~T}^{2} \mathrm{x}^{2} \alpha 0^{2}\left((5664+9 \mathrm{~T} \alpha 0(40+\mathrm{T} \alpha 0)) \alpha 1^{4}-\right.$

$\left.\left.128 \alpha 0(62+3 \mathrm{~T} \alpha 0) \alpha 1^{2} \alpha 2+1024 \alpha 0^{2} \alpha 2^{2}+2304 \alpha 0^{2} \alpha 1 \alpha 3\right)\right)+$ $x\left(28800 x^{6} \alpha 1^{4}-3360 T x^{4} \alpha 0 \alpha 1^{2}\left(3(28+T \alpha 0) \alpha 1^{2}-64 \alpha 0 \alpha 2\right)+\right.$ $100 \mathrm{~T}^{2} \mathrm{x}^{2} \alpha 0^{2}\left((4896+3 \mathrm{~T} \alpha 0(112+3 \mathrm{~T} \alpha 0)) \alpha 1^{4}-\right.$

$\left.384 \alpha 0(19+\mathrm{T} \alpha 0) \alpha 1^{2} \alpha 2+1024 \alpha 0^{2} \alpha 2^{2}+2304 \alpha 0^{2} \alpha 1 \alpha 3\right)-$

$T^{3} \alpha 0^{3}\left(15(4992+T \alpha 0(480+T \alpha 0(20+T \alpha 0))) \alpha 1^{4}-\right.$

$320 \alpha 0(504+T \alpha 0(44+3 T \alpha 0)) \alpha 1^{2} \alpha 2+$

$2304 \alpha 0^{2}(36+5 \mathrm{~T} \alpha 0) \alpha 1 \alpha 3+1024 \alpha 0^{2}$

$\left.\left.\left.\left((48+5 \mathrm{~T} \alpha 0) \alpha 2^{2}-72 \alpha 0 \alpha 4\right)\right)\right)\right)+$

$\log [K]\left(960 r^{6} T^{6} \alpha 1^{4}+28800 r^{5} T^{5} \times \alpha 1^{4}+201600 x^{6} \alpha 1^{4}-\right.$

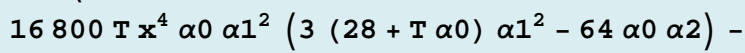

$240 \mathrm{r}^{4} \mathrm{~T}^{4} \alpha 1^{2}\left(-900 \mathrm{x}^{2} \alpha 1^{2}+\mathrm{T} \alpha 0\left((100+3 \mathrm{~T} \alpha 0) \alpha 1^{2}-64 \alpha 0 \alpha 2\right)\right)-$ $4800 \mathrm{r}^{3} \mathrm{~T}^{3} \times \alpha 1^{2}\left(-140 \mathrm{x}^{2} \alpha 1^{2}+\mathrm{T} \alpha 0\left((100+3 T \alpha 0) \alpha 1^{2}-64 \alpha 0 \alpha 2\right)\right)+$ $300 \mathrm{~T}^{2} \mathrm{x}^{2} \alpha 0^{2}\left((4896+3 \mathrm{~T} \alpha 0(112+3 \mathrm{~T} \alpha 0)) \alpha 1^{4}-\right.$

$\left.384 \alpha 0(19+\mathrm{T} \alpha 0) \alpha 1^{2} \alpha 2+1024 \alpha 0^{2} \alpha 2^{2}+2304 \alpha 0^{2} \alpha 1 \alpha 3\right)+$ $20 \mathrm{r}^{2} \mathrm{~T}^{2}\left(50400 \mathrm{x}^{4} \alpha 1^{4}-360 \mathrm{~T} \mathrm{x}^{2} \alpha 0 \alpha 1^{2}\right.$

$\left((280+9 T \alpha 0) \alpha 1^{2}-192 \alpha 0 \alpha 2\right)+$

$T^{2} \alpha 0^{2}\left((6688+9 T \alpha 0(40+T \alpha 0)) \alpha 1^{4}-\right.$

$\left.\left.384 \alpha 0(22+T \alpha 0) \alpha 1^{2} \alpha 2+1024 \alpha 0^{2} \alpha 2^{2}+2304 \alpha 0^{2} \alpha 1 \alpha 3\right)\right)+$ $40 \mathrm{r} \times\left(18144 \mathrm{x}^{4} \alpha 1^{4}-840 \mathrm{~T} \mathrm{x^{2 }} \alpha 0 \alpha 1^{2}\right.$

$\left((88+3 T \alpha 0) \alpha 1^{2}-64 \alpha 0 \alpha 2\right)+$

$5 \mathrm{~T}^{2} \alpha 0^{2}\left((5664+9 \mathrm{~T} \alpha 0(40+\mathrm{T} \alpha 0)) \alpha 1^{4}-\right.$

$\left.\left.128 \alpha 0(62+3 \mathrm{~T} \alpha 0) \alpha 1^{2} \alpha 2+1024 \alpha 0^{2} \alpha 2^{2}+2304 \alpha 0^{2} \alpha 1 \alpha 3\right)\right)-$

$T^{3} \alpha 0^{3}\left(15(4992+T \alpha 0(480+T \alpha 0(20+T \alpha 0))) \alpha 1^{4}-\right.$ 


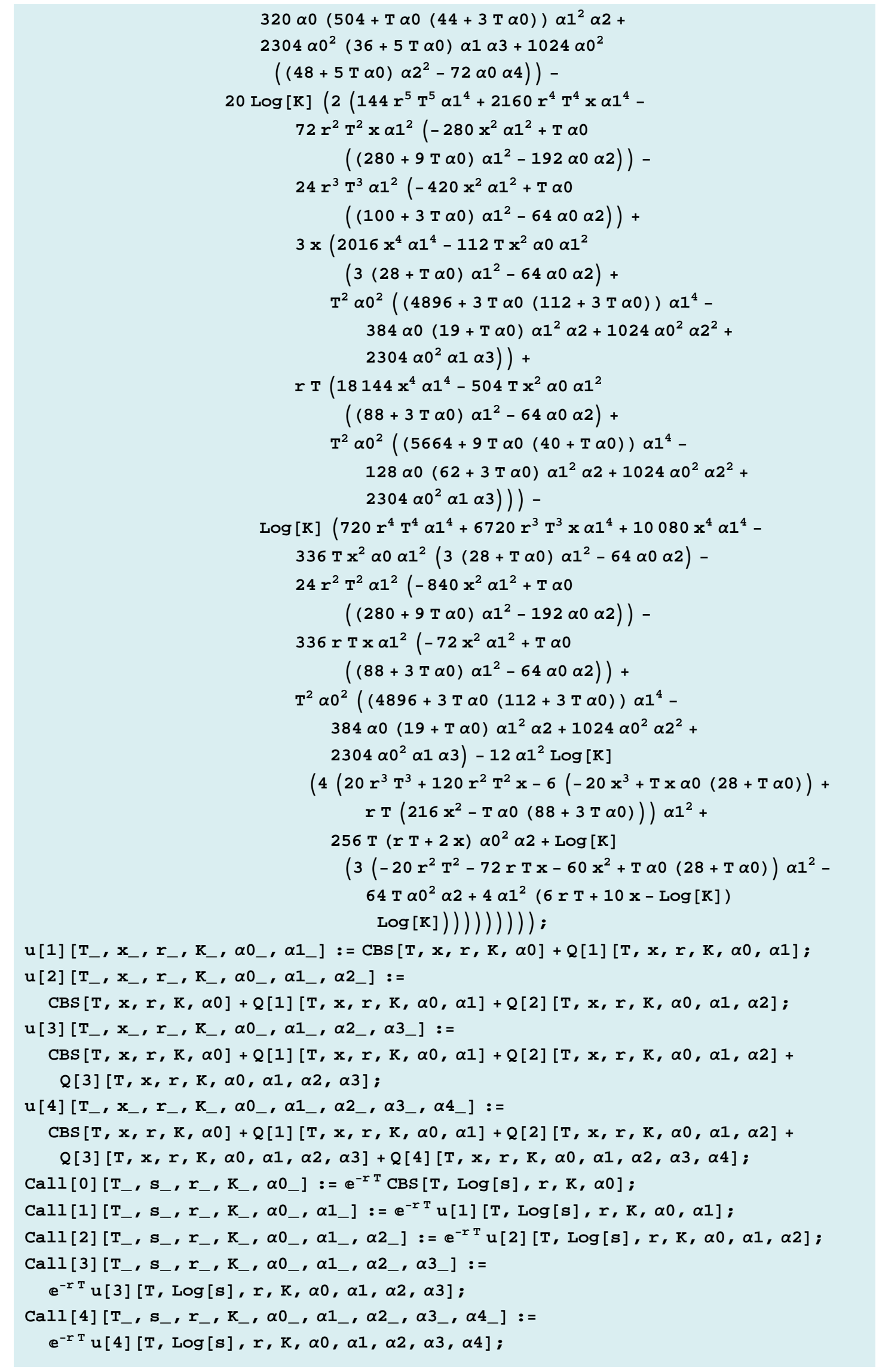


1. Enter item1numbered text here.

Enter item1paragraph text here.

a. Enter item2numbered text here.

Enter item2paragraph text here.

i. Enter item3numbered text here.

Enter item3paragraph text here.

Enter text here. Enter numbered equation for display in a separate cell below:

$$
\int x d x+\sqrt{z}
$$

- Enter item1 text here.

Enter item1paragraph text here.

- Enter item2 text here.

Enter item2paragraph text here.

- Enter item3 text here.

Enter item3paragraph text here.

\section{Example: the CEV model}

In the CEV model the volatility function in (1) takes the form

$$
\sigma\left(S_{t}\right)=\sigma S_{t}^{\beta}
$$

where $\beta \in[0,1]$ and $\sigma$ is a positive constant. First of all, we compute the constants $\alpha_{n}$ appearing in the expansion:

$$
\begin{aligned}
& \operatorname{vol}\left[s_{-}\right]:=\sigma s^{\beta-1} ; \\
& \alpha 0\left[x_{-}, \beta_{-}, \sigma_{-}\right]:=\operatorname{vol}\left[e^{x}\right]^{2} / / \text { FullSimplify // Evaluate } \\
& \alpha 1\left[x_{-}, \beta_{-}, \sigma_{-}\right]:=\partial_{x}\left(\frac{1}{2} \operatorname{vol}\left[e^{x}\right]^{2}\right) / / \text { FullSimplify // Evaluate } \\
& \alpha 2\left[x_{-}, \beta_{-}, \sigma_{-}\right]:=\frac{1}{2} \partial_{x, x}\left(\frac{1}{2} \operatorname{vol}\left[e^{x}\right]^{2}\right) / / \text { FullSimplify // Evaluate } \\
& \alpha 3\left[x_{-}, \beta_{-}, \sigma_{-}\right]:=\frac{1}{6} \partial_{x, x, x}\left(\frac{1}{2} \operatorname{vol}\left[e^{x}\right]^{2}\right) / / \text { FullSimplify // Evaluate } \\
& \alpha 4\left[x_{-}, \beta_{-}, \sigma_{-}\right]:=\frac{1}{24} \partial_{x, x, x, x}\left(\frac{1}{2} \operatorname{vol}\left[e^{x}\right]^{2}\right) / / \text { FullSimplify // Evaluate }
\end{aligned}
$$

We can now define the function $\Gamma \operatorname{Cev}[n]\left[\mathrm{s}_{-}, \mathrm{y}_{-}, \mathrm{T}_{-}, \mathrm{r}_{-}, \sigma_{-}, \beta_{-}\right]$that rappresents the $n$-th order approximation formulas of the density

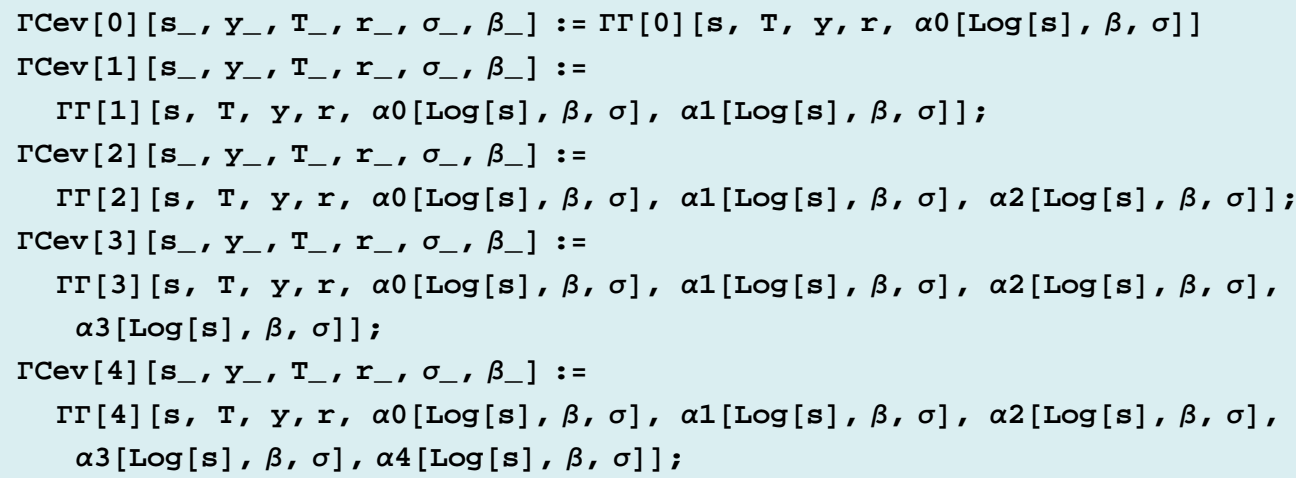

and the function CevCall $[n]\left[\mathrm{T}_{-}, \mathrm{s}_{-}, \mathrm{K}_{-}, \mathrm{r}_{-}, \sigma_{-}, \beta_{-}\right]$that rappresent the $n$-th order approximation formula for the Call price 


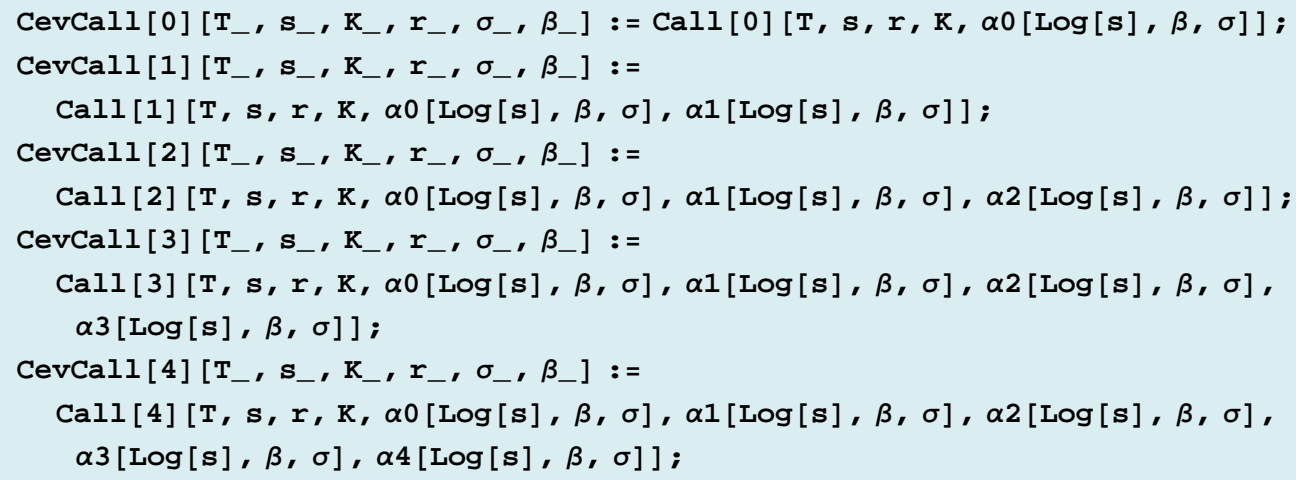

\section{Numerical tests}

In this section we report some the numerical tests of Section 4 in [PP2011].

\section{Density approximations}

In this subsection we only consider the case $r=0$. We define the functions $\Gamma$ Bessel, $\Gamma$ LH and Gamma2Hagan that are the exact CEV density, the approximation by Henry-Labordère and by Hagan-Woodward (that is, the approximation obtained by the above procedure without the log-chage of variables) respectively.

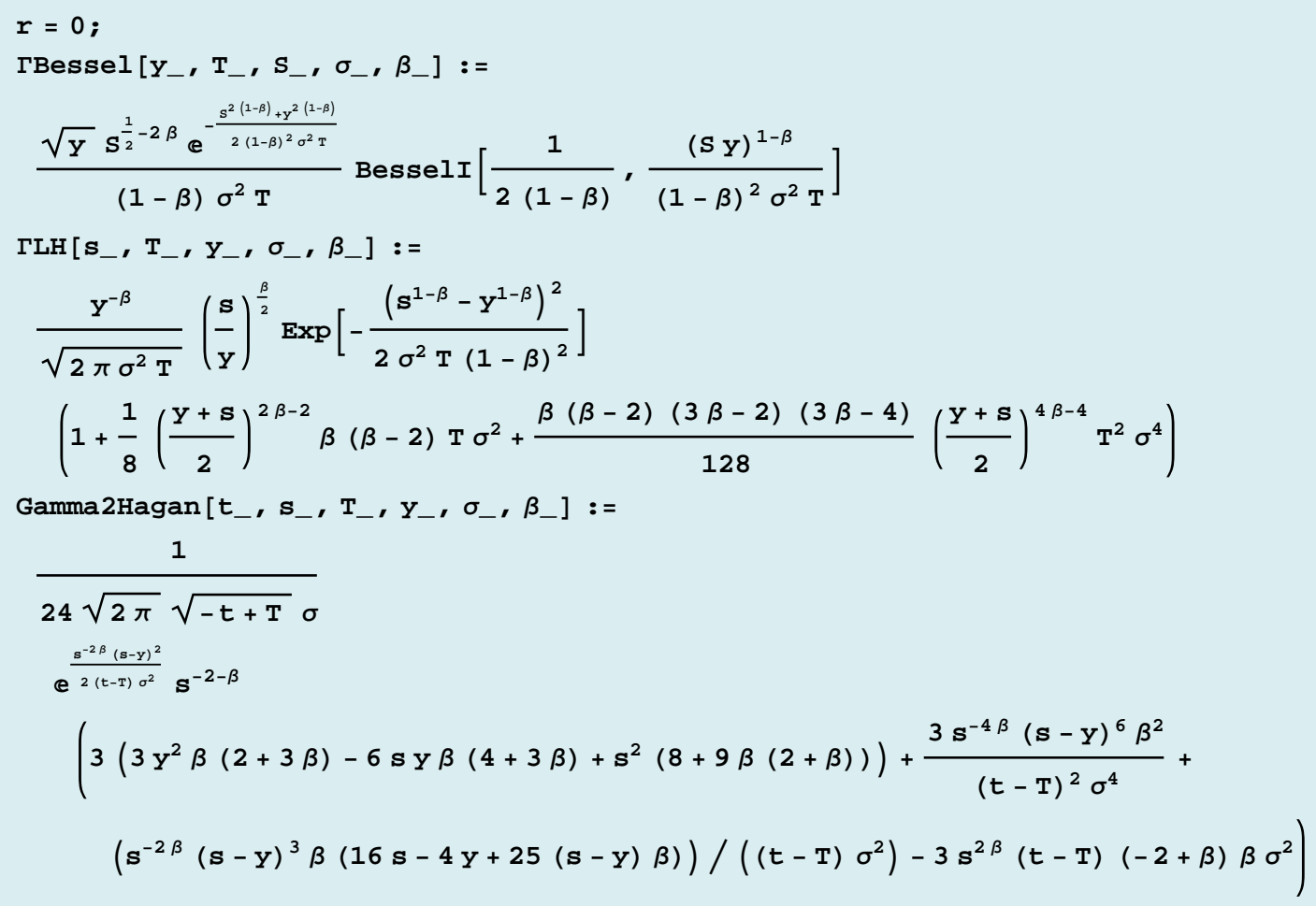

We plot the ГBessel (thick line), ГLH (dot-dashed line), Gamma2Hagan (dotted line) and our 4-th order approximation ГCev[4] (dashed line) for $S_{0}=1$ and different maturities: cf. Figures 4 and 5 in [PP2011]. 

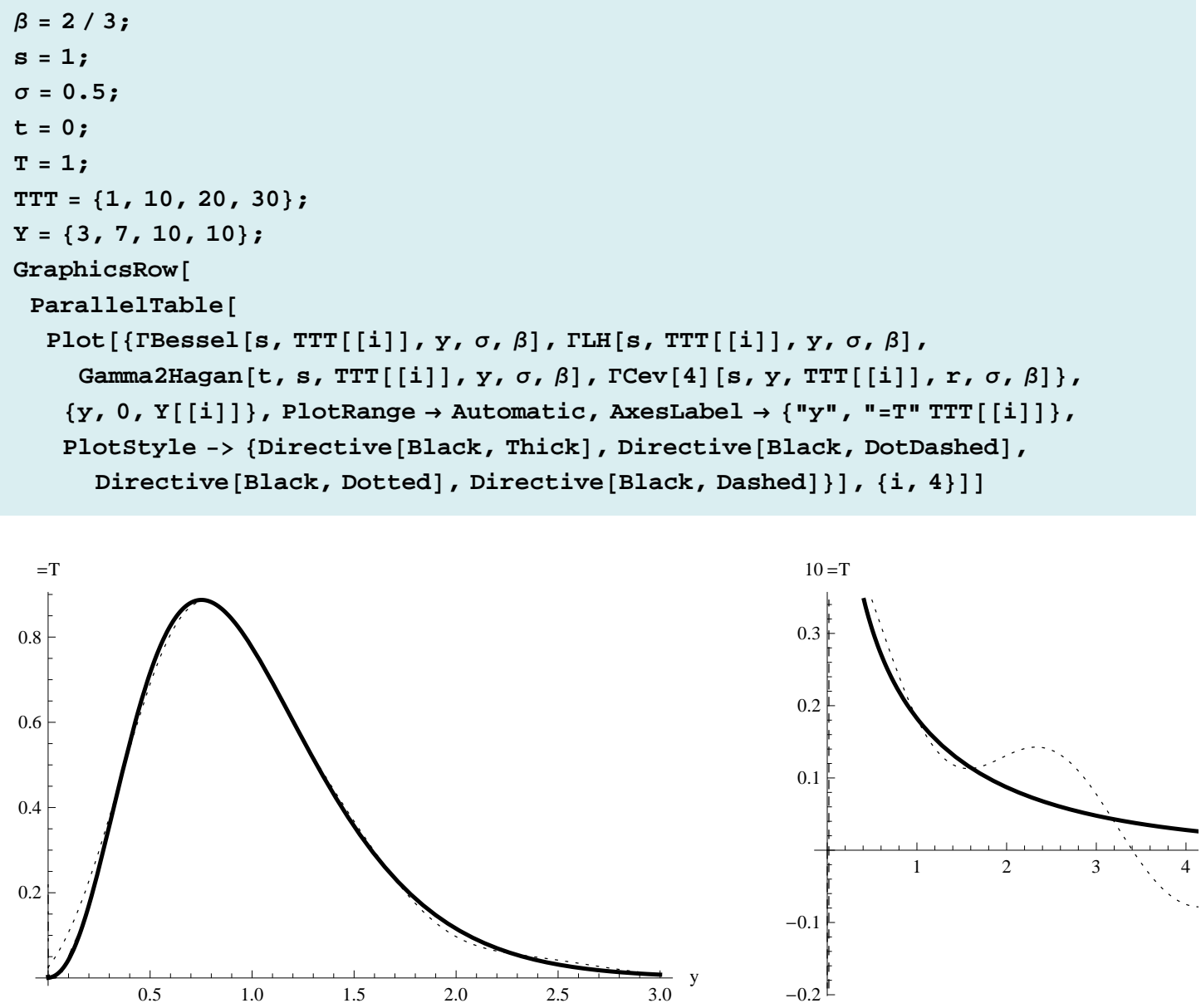

Next we plot the value of the integral of the Gamma2Hagan and ГLH approximations: cf. Figures 6 and 7 in [PP2011]. 

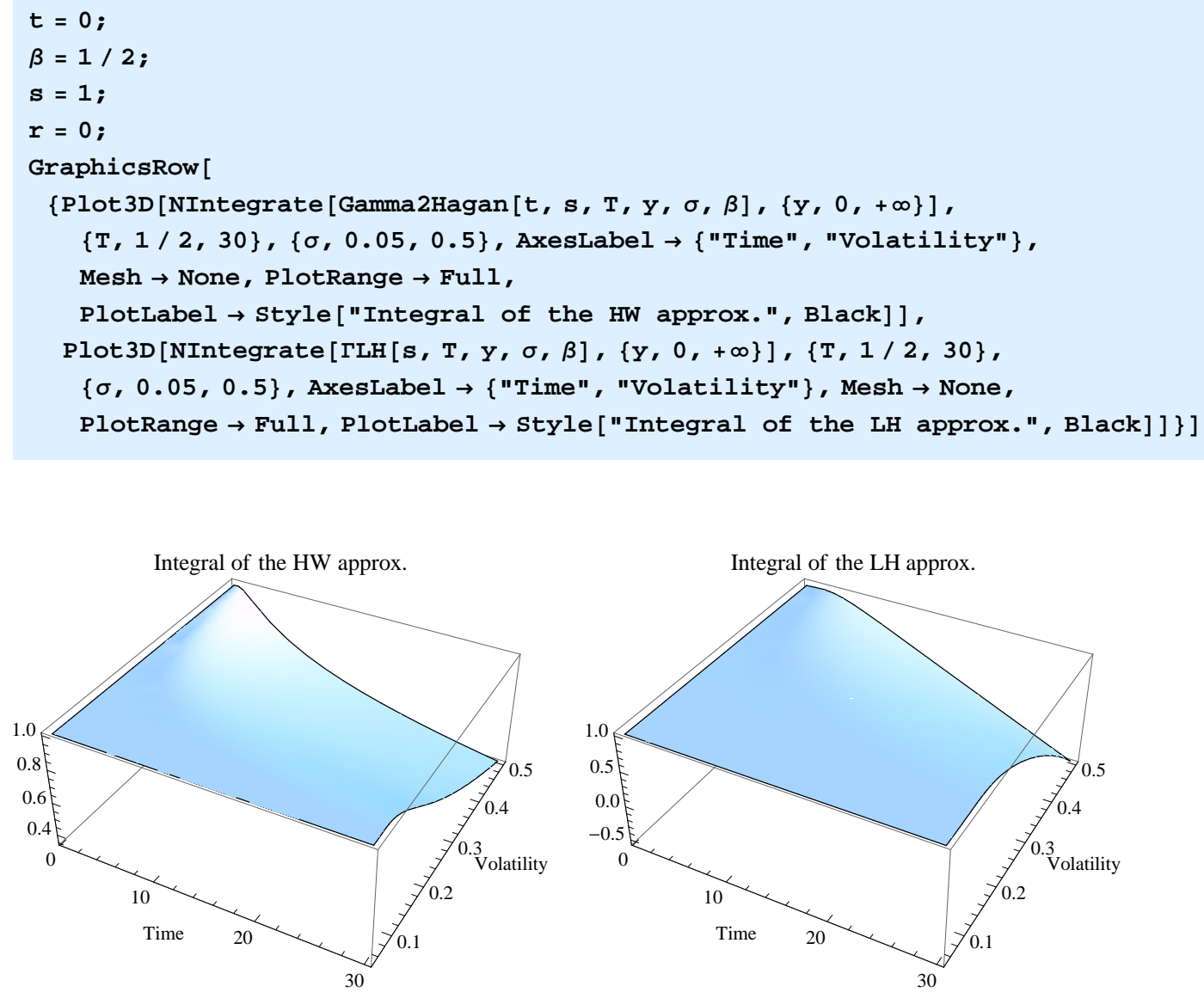

\section{Call price approximations}

Below, we define the Call price obtained by convolution with $\Gamma$ Bessel:

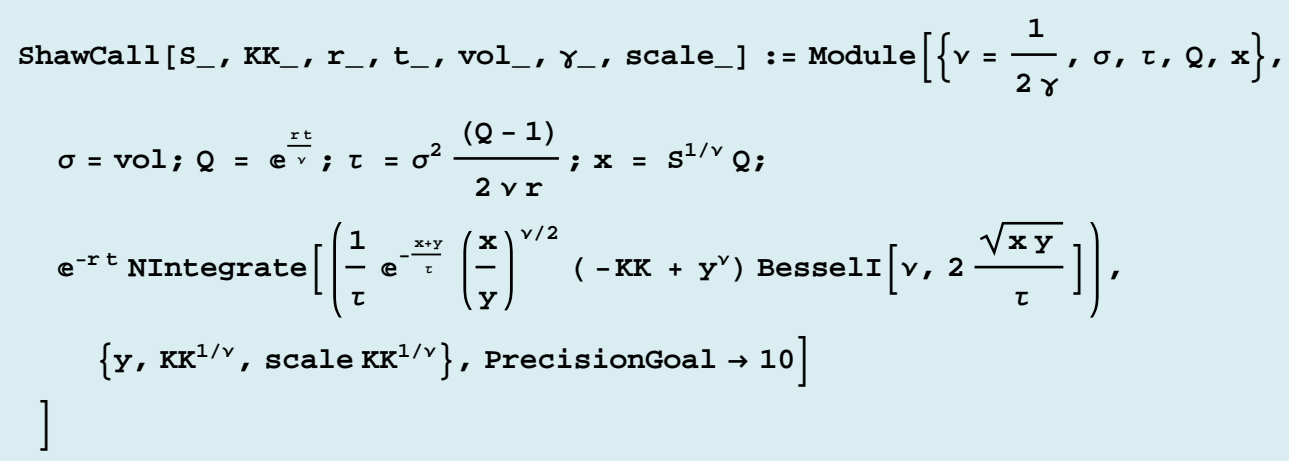

We noe define the function CallHagan2 that is the approximation of the CEV-Call price given by the 2-nd order implied volatility approximation by Hagan and Woodward. 


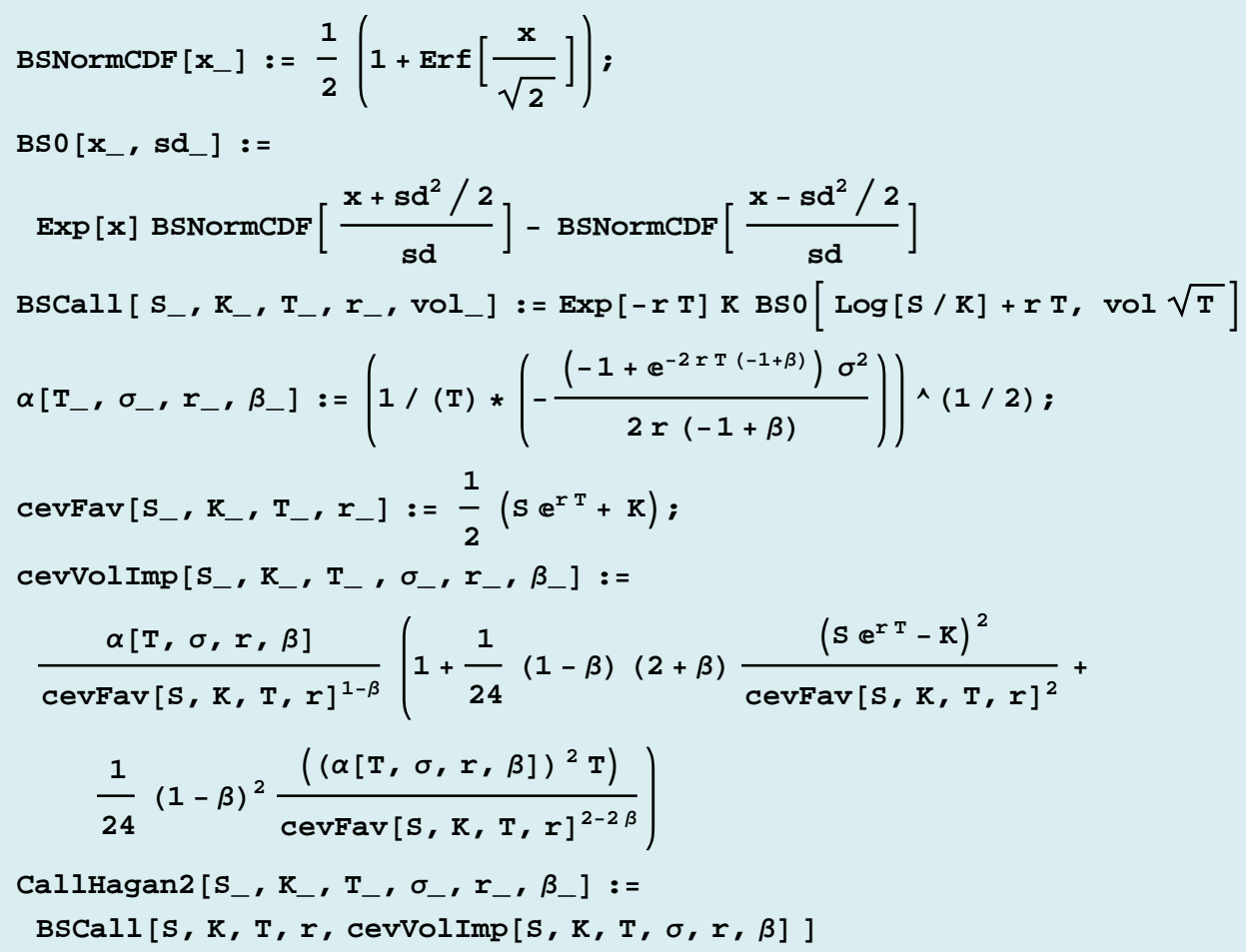

Here we show that the Put-Call parity is violated by the HW and LH approximations: cf. Tables 1 and 2 in [PP2011]. 


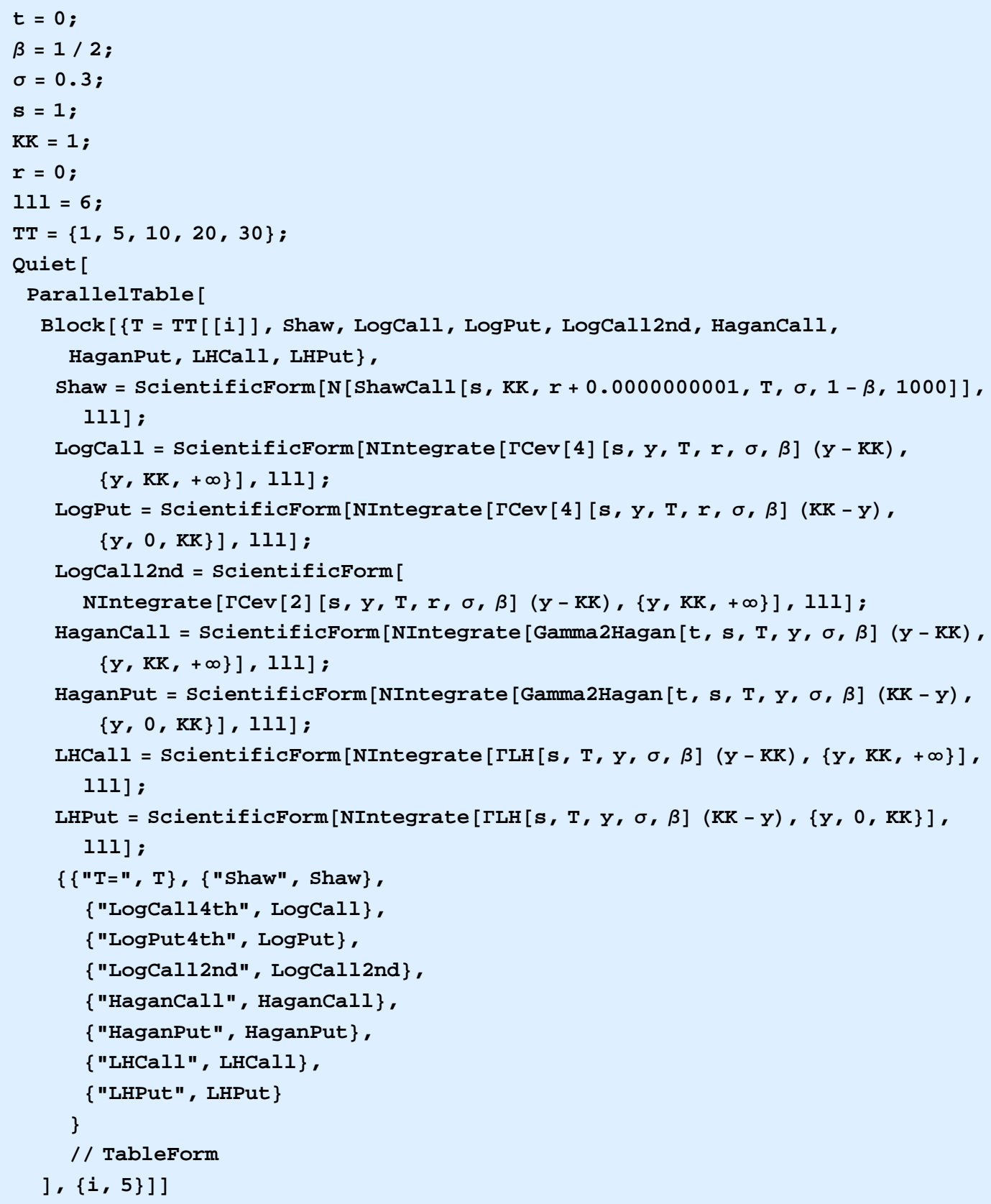




\begin{tabular}{|c|c|c|c|}
\hline $\mathrm{T}=$ & 1 & & \\
\hline Shaw & $1.19345 \times 10^{-1}$ & & \\
\hline LogCall4th & $1.19345 \times 10^{-1}$ & & \\
\hline LogPut 4th & $1.19345 \times 10^{-1}$ & & \\
\hline LogCall2nd & $1.19344 \times 10^{-1}$ & & \\
\hline HaganCall & $1.19346 \times 10^{-1}$ & & \\
\hline HaganPut & $1.19174 \times 10^{-1}$ & & \\
\hline LHCall & $1.19359 \times 10^{-1}$ & & \\
\hline LHPut & $1.1939 \times 10^{-1}$ & & \\
\hline$T=$ & 5 & $\mathrm{~T}=$ & 10 \\
\hline Shaw & $2.63769 \times 10^{-1}$ & Shaw & $3.67286 \times 10^{-1}$ \\
\hline LogCall4th & $2.63768 \times 10^{-1}$ & LogCall4th & $3.67295 \times 10^{-1}$ \\
\hline LogPut 4th & $2.63768 \times 10^{-1}$ & LogPut 4 th & $3.67295 \times 10^{-1}$ \\
\hline LogCall2nd & $2.63737 \times 10^{-1}$ & LogCal12nd & $3.67201 \times 10^{-1}$ \\
\hline HaganCall & $2.63855 \times 10^{-1}$ & HaganCall & $3.67825 \times 10^{-1}$ \\
\hline HaganPut & $2.40181 \times 10^{-1}$ & HaganPut & $2.39639 \times 10^{-1}$ \\
\hline LHCall & $2.64278 \times 10^{-1}$ & LHCall & $3.69572 \times 10^{-1}$ \\
\hline LHPut & $2.70363 \times 10^{-1}$ & LHPut & $3.53664 \times 10^{-1}$ \\
\hline $\mathrm{T}=$ & 20 & $\mathrm{~T}=$ & 30 \\
\hline Shaw & $5.01275 \times 10^{-1}$ & Shaw & $5.89194 \times 10^{-1}$ \\
\hline LogCall4th & $5.01915 \times 10^{-1}$ & LogCall4th & $5.91281 \times 10^{-1}$ \\
\hline LogPut 4 th & $5.01915 \times 10^{-1}$ & LogPut 4 th & $5.91281 \times 10^{-1}$ \\
\hline LogCal12nd & $5.02073 \times 10^{-1}$ & LogCal12nd & $5.92962 \times 10^{-1}$ \\
\hline HaganCall & $5.0513 \times 10^{-1}$ & HaganCall & $6.00219 \times 10^{-1}$ \\
\hline HaganPut & $1.97434 \times 10^{-1}$ & HaganPut & $1.64068 \times 10^{-1}$ \\
\hline LHCall & $5.11899 \times 10^{-1}$ & LHCall & $6.14735 \times 10^{-1}$ \\
\hline LHPut & $3.14135 \times 10^{-1}$ & LHPut & $1.87179 \times 10^{-1}$ \\
\hline
\end{tabular}

Finally, we define the relative errors for the previous approximations of Call prices

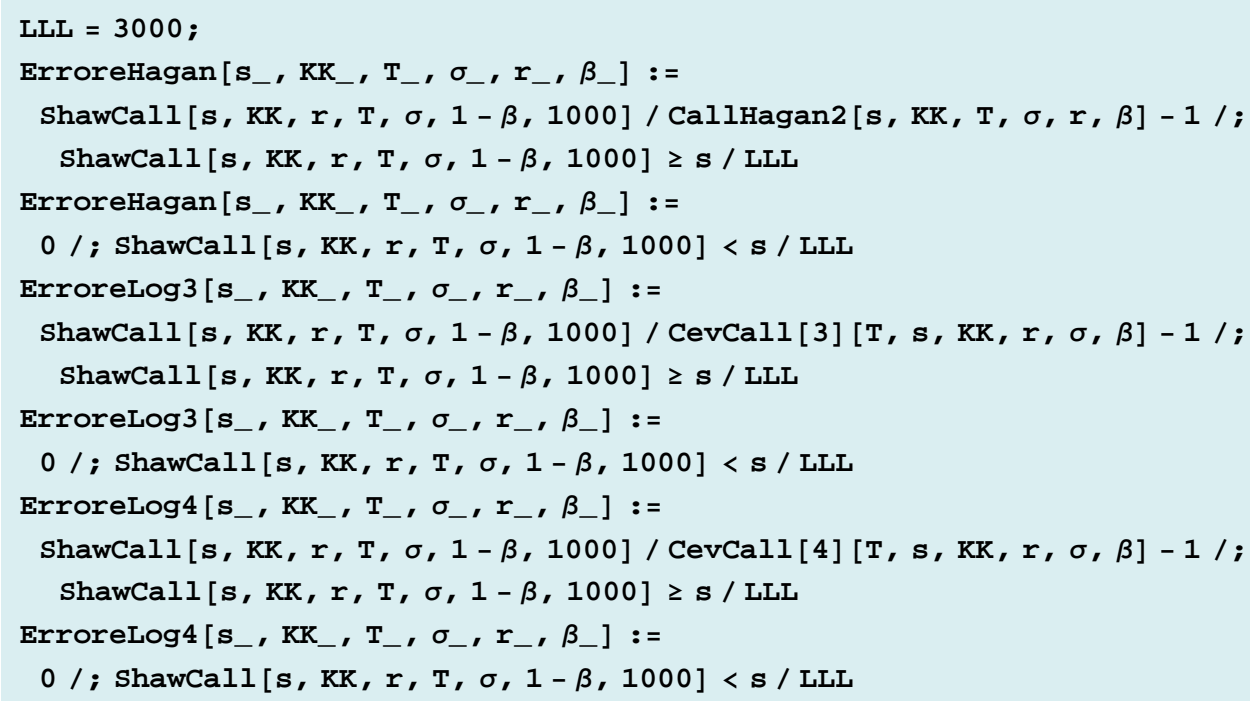

Figure 8 in [PP2011] 


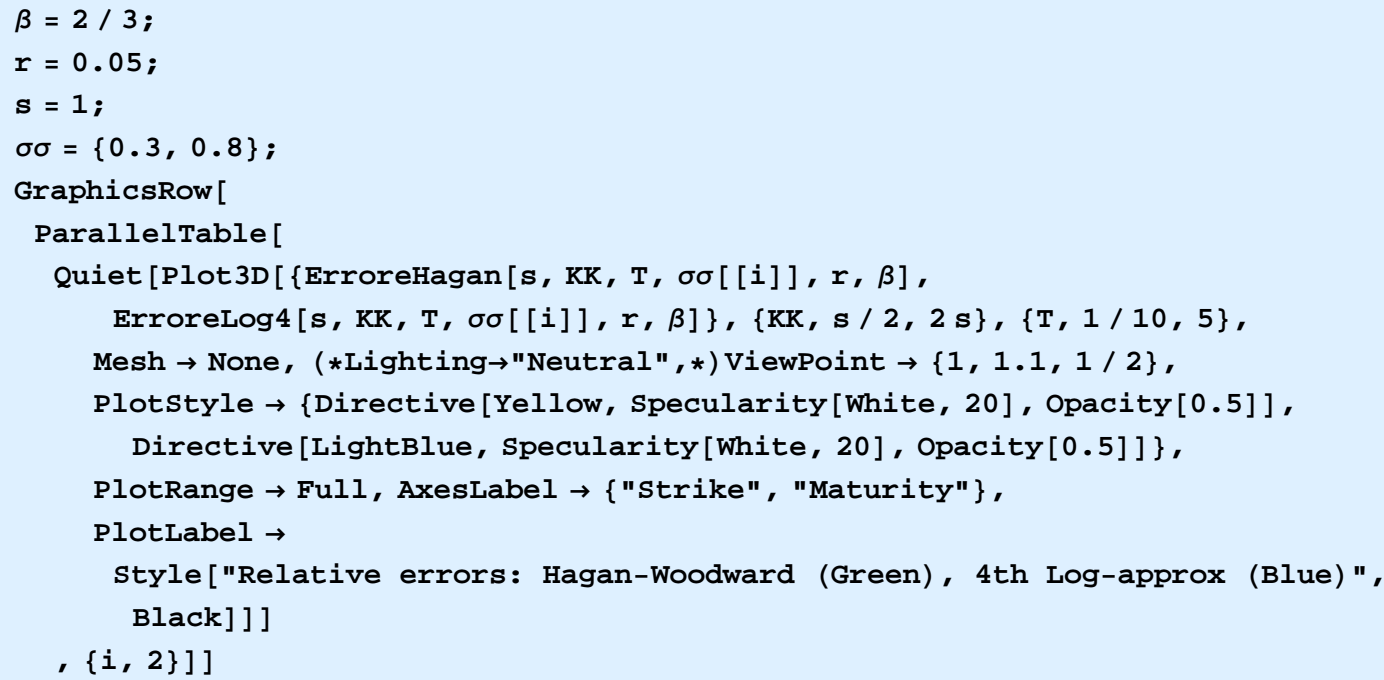

ive errors: Hagan-Woodward (Green), 4th Log-approx (

ive errors: Hagan-Woodward (Green), 4th Log-approx (
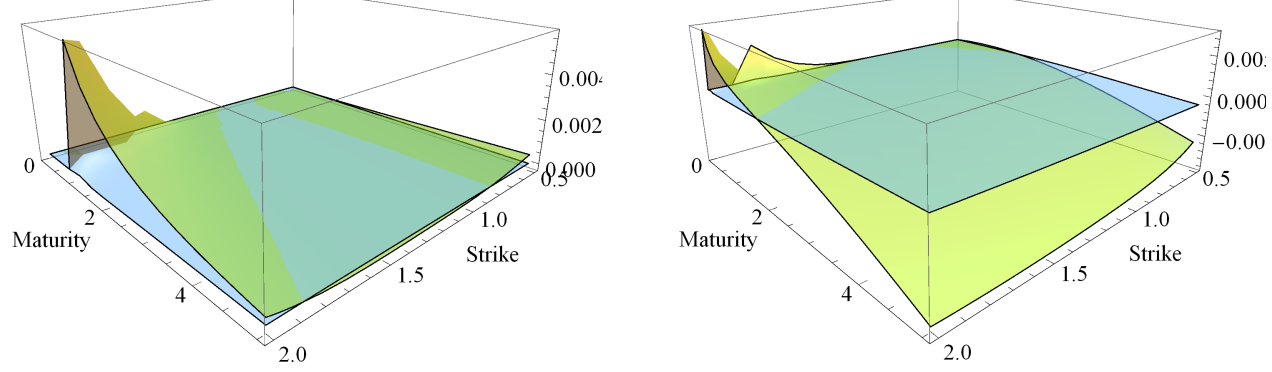

Figure 9 in [PP2011] 


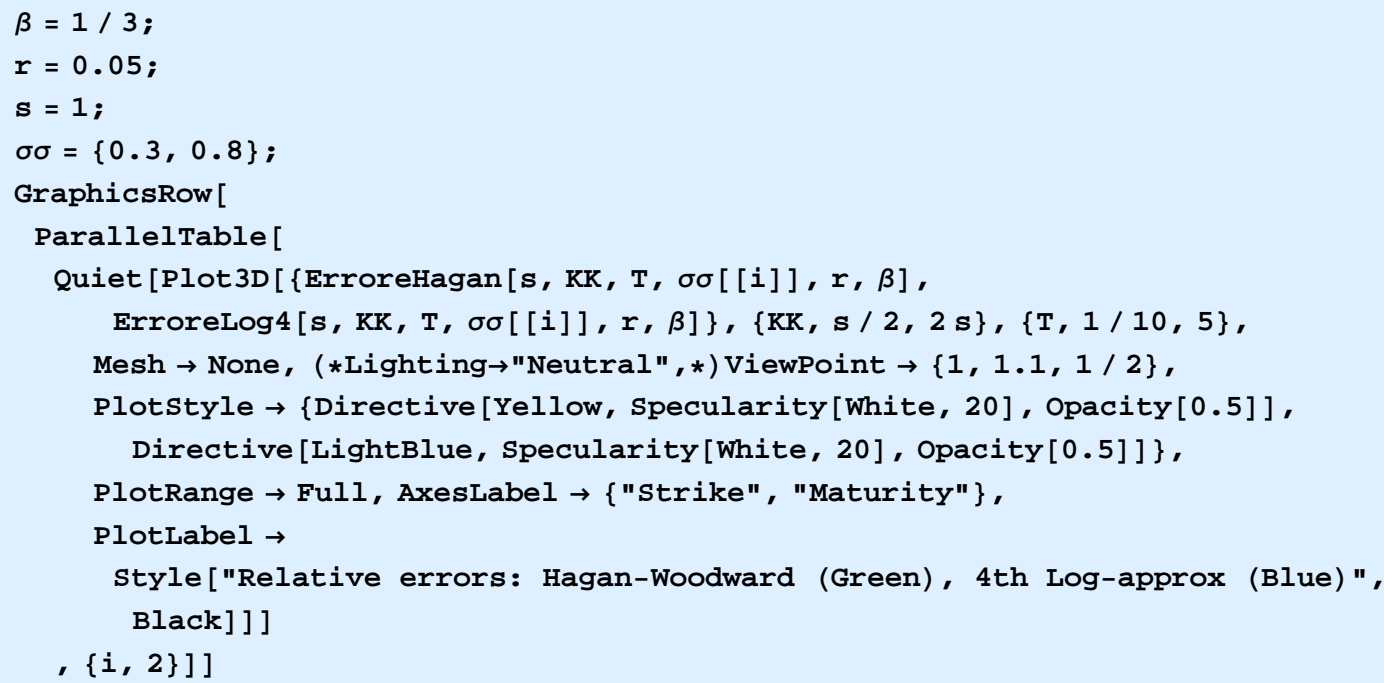

ive errors: Hagan-Woodward (Green), 4th Log-approx ( ive errors: Hagan-Woodward (Green), 4th Log-approx (
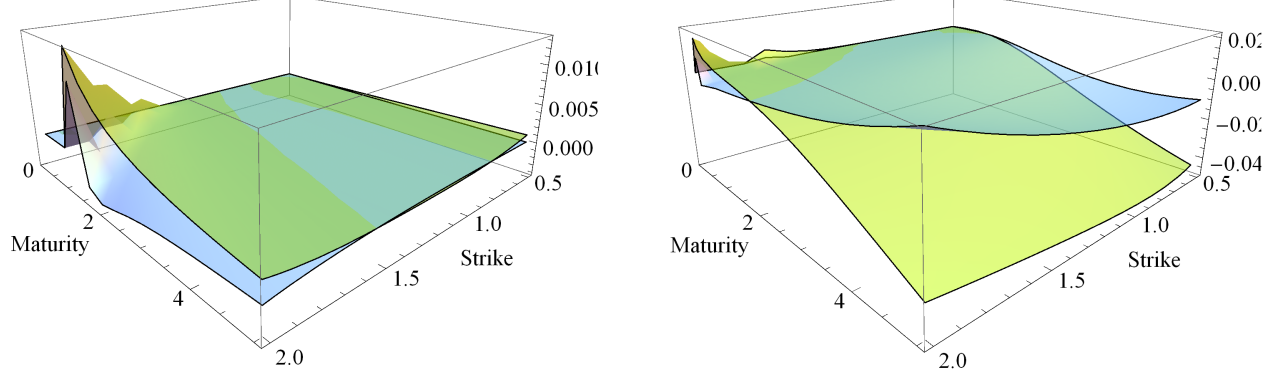

Figure 10 in [PP2011] 


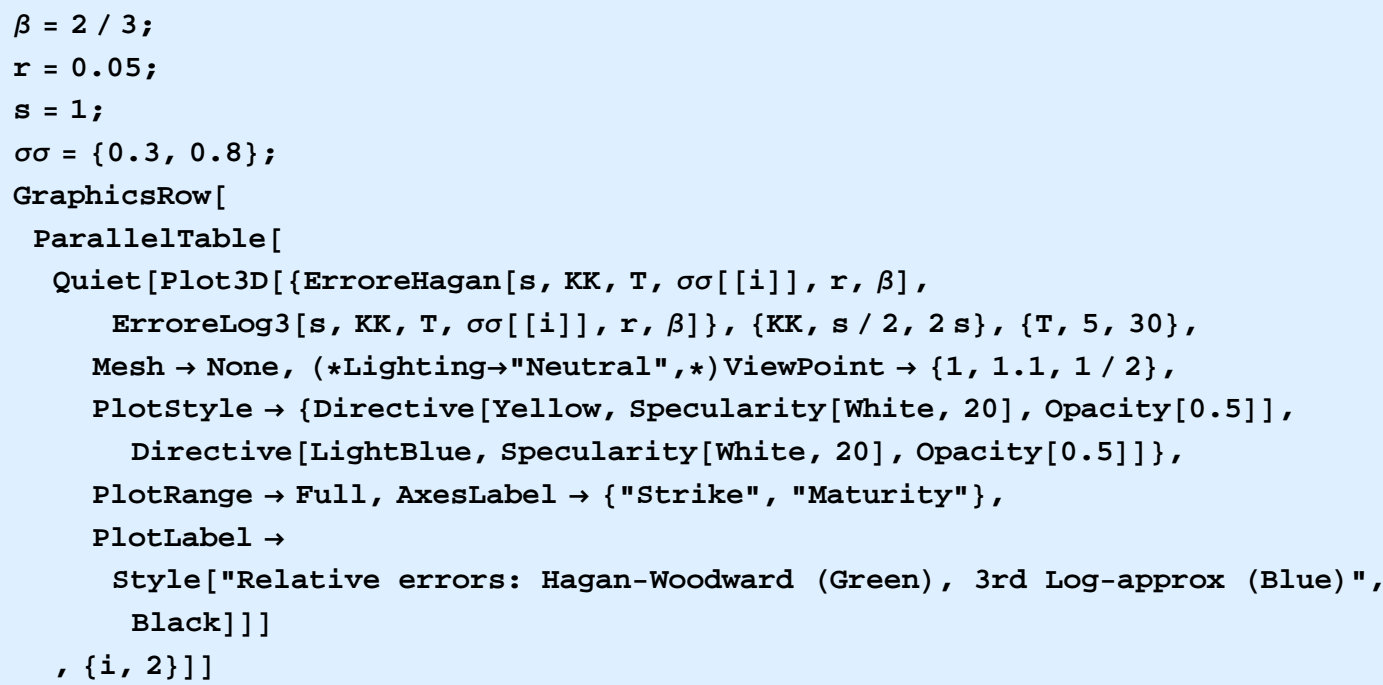

ive errors: Hagan-Woodward (Green), 3rd Log-approx ( ive errors: Hagan-Woodward (Green), 3rd Log-approx (
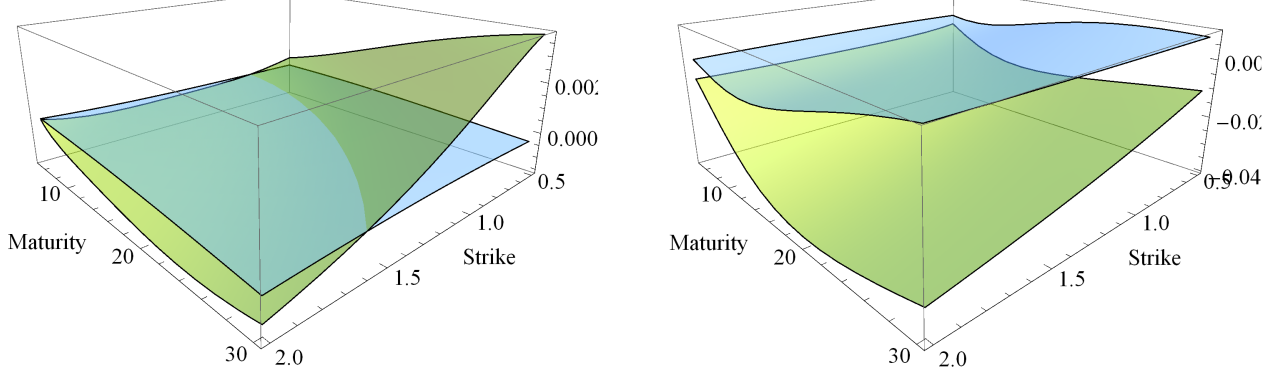

Figure 11 in [PP2011] 


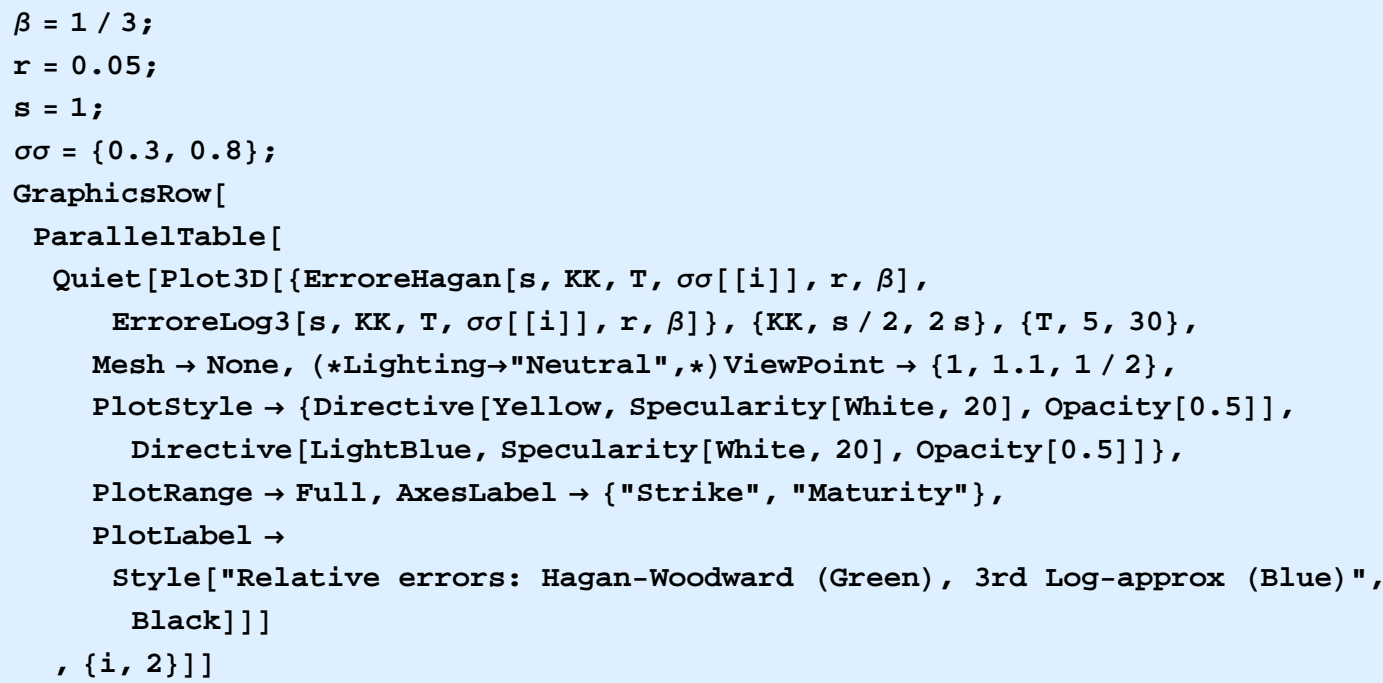

ve errors: Hagan-Woodward (Green), 3rd Log-approx (

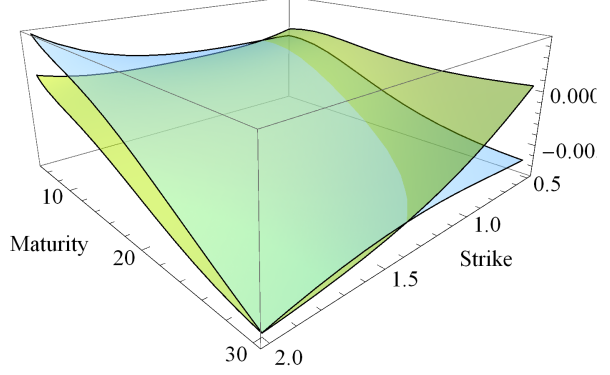

ive errors: Hagan-Woodward (Green), 3rd Log-approx (

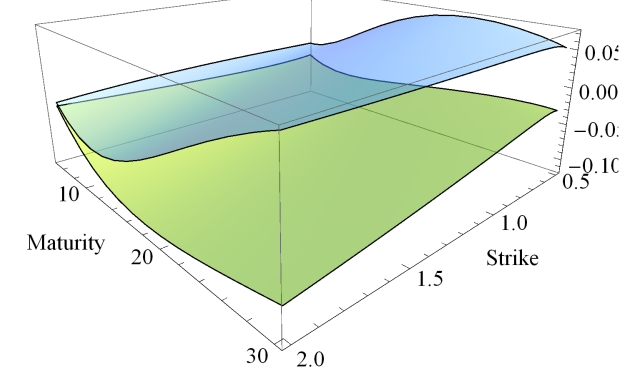

